\title{
Changes in the Sclerotinia sclerotiorum transcriptome during infection of Brassica napus
}

\author{
Shirin Seifbarghi ${ }^{1,2}$, M. Hossein Borhan ${ }^{1}$, Yangdou Wei ${ }^{2}$, Cathy Coutu', Stephen J. Robinson ${ }^{1}$ \\ and Dwayne D. Hegedus ${ }^{1,3^{*}}$
}

\begin{abstract}
Background: Sclerotinia sclerotiorum causes stem rot in Brassica napus, which leads to lodging and severe yield losses. Although recent studies have explored significant progress in the characterization of individual S. sclerotiorum pathogenicity factors, a gap exists in profiling gene expression throughout the course of S. sclerotiorum infection on a host plant. In this study, RNA-Seq analysis was performed with focus on the events occurring through the early $(1 \mathrm{~h})$ to the middle (48 h) stages of infection.

Results: Transcript analysis revealed the temporal pattern and amplitude of the deployment of genes associated with aspects of pathogenicity or virulence during the course of $\mathrm{S}$. sclerotiorum infection on Brassica napus. These genes were categorized into eight functional groups: hydrolytic enzymes, secondary metabolites, detoxification, signaling, development, secreted effectors, oxalic acid and reactive oxygen species production. The induction patterns of nearly all of these genes agreed with their predicted functions. Principal component analysis delineated gene expression patterns that signified transitions between pathogenic phases, namely host penetration, ramification and necrotic stages, and provided evidence for the occurrence of a brief biotrophic phase soon after host penetration.

Conclusions: The current observations support the notion that S. sclerotiorum deploys an array of factors and complex strategies to facilitate host colonization and mitigate host defenses. This investigation provides a broad overview of the sequential expression of virulence/pathogenicity-associated genes during infection of B. napus by $S$. sclerotiorum and provides information for further characterization of genes involved in the S. sclerotiorum-host plant interactions.
\end{abstract}

Keywords: Sclerotinia sclerotiorum, Brassica napus, Infection, Transcriptome, Necrosis, Effectors, Hydrolytic enzymes, Secondary metabolites, Oxalic acid

\section{Background}

Sclerotinia sclerotiorum (Lib.) de Bary causes one of the most devastating diseases of canola, stem rot. This pathogen has a wide host-range and can infect more than 400 plant species, including many other important crop plants [1]. This fungus was long considered to be a prototypical necrotrophic pathogen whereby immediately upon host cuticle penetration a highly aggressive pathogenic

\footnotetext{
* Correspondence: Dwayne.Hegedus@agr.gc.ca

${ }^{1}$ Agriculture and Agri-Food Canada, 107 Science Place, Saskatoon, SK S7N 0X2, Canada

${ }^{3}$ Department of Food and Bioproduct Sciences, University of Saskatchewan Saskatoon, Canada

Full list of author information is available at the end of the article
}

phase ensues where acids and hydrolytic enzymes are liberated in advance of the invading mycelia with a trailing saprophytic phase that supports sclerotia formation [2]. Recent studies, however, have provided evidence for a brief biotrophic phase occurring within the apoplastic space immediately after cuticle penetration and the pathogen may, therefore, be more accurately classified as a hemi-biotroph [3]. Transition between these various developmental and pathogenic phases is governed by physical and metabolic cues including detection of contact with hard surfaces [4], glucose levels [5], cAMP levels [6], $\mathrm{pH}$ [7] and oxidative stress [8]. Communication between the associated 
signaling pathways is critical and involves numerous protein kinases [9-11] and phosphatases [12, 13].

Much of the research on the molecular mechanisms of virulence in S. sclerotiorum has focused on oxalic acid (OA) which plays various roles during several stages of the infection [14]. OA suppresses the oxidative burst and callose deposition during the early stages of the infection [15]. Suppression of host defenses by OA during the biotrophic phase is thought to allow sufficient time for the pathogen to establish itself in the host as a prelude to mycelial ramification [3]. Subsequently, OA induces the production of host reactive oxygen species (ROS), which in turn leads to host cell death [16]. As a central player in S. sclerotiorum pathogenesis, it is not surprising that plants expressing oxalate-degrading enzymes exhibit increased resistance to this pathogen [17]. Pathogen-derived ROS generated through NADPH oxidase activity are associated with appressoria formation and sclerotial development, as well as oxalic acid synthesis [8]. Catalase (SCat1) [18] and superoxide dismutase (SsSodI) [19] appear to modulate the deleterious effects of these compounds internally. The apoptosis inhibitor, BAX inhibitor-1 (SsBI1), is also required for full virulence and was postulated to prevent hyphal apoptosis resulting from exposure to host-derived ROS [20].

The production of numerous types of hydrolytic and cell wall degrading enzymes (CWDE) facilitates host cuticle penetration, lesion expansion and tissue maceration $[4,21,22]$. Although pectinolytic CWDEs, such as polygalacturonases (SsPG1, SsPG3, SsPG5 and SsPG6), have captured more attention as the main group of hydrolytic enzymes involved in S. sclerotiorum virulence [23], non-pectinolytic enzymes like proteases, cellulases and glucoamylases also contribute to the infection process in this fungus [21].

Several other factors are known to contribute to $S$. sclerotiorum pathogenicity and host interactions. Both $\gamma$-glutamyl transpeptidase (SsGgt1) and compound appressorium formation-related protein 1 (Ss-Caf1) influence the production of compound appressoria and subsequent host penetration, but also development of sclerotia $[24,25]$. A secreted integrin-like protein (SSITL) inhibits the deployment of plant defenses through the jasmonic/ethylene signaling pathways [26] and a chorismate mutase $(S s C m 1)$ may function similarly to suppress plant defense responses during the biotrophic phase [27]. Host chemical defenses may be inactivated by inducible detoxification systems [28], while other proteins, such as SsPemG1 (protein elicitor from Magnaporthe grisea), are recognized by the host and induce defenses [29]. SsNEP1 and SsNEP2 encode necrosis and ethylene-inducing like proteins (NLP), which induce necrosis in host tissues [30], as does cutinase [31]. A gene (SS1G_00263, ssv263) encoding a hypothetical protein with unknown mode of action is a virulence factor in $S$. sclerotiorum [32].

Transcriptomics and proteomics approaches have been used to gain insight into molecular interaction of $S$. sclerotiorum with its various hosts. Expressed sequence tag (EST) analysis was used to identify genes associated with pathogenesis by comparing the transcriptome of $S$. sclerotiorum grown on artificial medium to that during infection of Brassica napus [33]. A similar approach was used to identify genes expressed during different stages of S. sclerotiorum development on this host [34], which was later supported by proteomics analysis [35]. Subsequently, microarray [36] and RNA-Seq analysis [37] was used to explore the $B$. napus responses to $S$. sclerotiorum. The release of the $S$. sclerotiorum genome sequence [38] in combination with next generation sequencing has allowed for in-depth analysis of the S. sclerotiorum pea [39], S. sclerotiorum - Phaseolus vulgaris [40] and S. homoeocarpa - creeping bentgrass [41] pathosystems. Proteomic analysis of exudates from liquid cultures has identified several secreted proteins that may be involved in aspects of pathogenesis [42]. Bioinformatic studies revealed that $S$. sclerotiorum has the potential to secrete a large number of proteins, many of which have the potential to influence host-pathogen interactions [43, 44].

While significant progress has been made in the characterization of individual S. sclerotiorum virulence and pathogenicity factors, a gap exists in our understanding of how the transcriptome is deployed throughout the course of S. sclerotiorum infection on a host plant. In this study, we used RNA-Seq analysis to comprehensively catalogue genes that were expressed and up-regulated during infection of $B$. napus, with a particular focus on the events occurring early in the infection. This work provided new insight into S. sclerotiorum pathogenesis through examination of the sequential expression of virulence and pathogenicity genes during infection establishment.

\section{Methods \\ Biological materials and disease assay}

S. sclerotiorum isolate 1980 (Ss1980) was used in this study as the genome sequence of this strain is available [38]. The doubled haploid B. napus cultivar DH12075 for which a genome sequence is available (Parkin, unpublished) was used as the host plant. Ss1980 was grown on minimal salts-glucose (MS-Glu: $2 \mathrm{~g} / \mathrm{L}$ $\mathrm{NH}_{4} \mathrm{NO}_{3}, 1 \mathrm{~g} / \mathrm{L} \mathrm{KH_{2 }} \mathrm{PO}_{4}, 0.1 \mathrm{~g} / \mathrm{L} \mathrm{MgSO}_{4} \cdot 7 \mathrm{H} 2 \mathrm{O}, 0.5$ $\mathrm{g} / \mathrm{L}$ yeast extract, $3 \mathrm{~g} / \mathrm{L}$ DL-malic acid, $1 \mathrm{~g} / \mathrm{L} \mathrm{NaOH}$, supplemented with $1 \%$ glucose) medium and mycelia were used for inoculation as described earlier [23]. One gram of mycelia (wet weight) was spread over a $5-\mathrm{cm}$ diameter circle on a detached leaf of a four week old plant and incubated in a sealed and humidified tray at room 
temperature. The experiment was conducted with three biological replicates. Samples collected from the fungal isolate grown in culture and on plants at 1, 3, 6, 12, 24 and $48 \mathrm{~h}$ post-inoculation (hpi) were subjected to RNA-Seq analysis.

\section{RNA extraction, library preparation and Illumina sequencing}

Fungal mats and the infected plant tissues beneath it were flash-frozen in liquid nitrogen and stored at $-80{ }^{\circ} \mathrm{C}$. The samples were ground to a fine powder with an RNAse-free mortar and pestle precooled with liquid nitrogen. Total RNA was extracted using an Illustra RNAspin mini RNA isolation kit (Illumina, San Diego, USA). RNA quantity and quality was assessed using a Qubit fluorometry assay (Invitrogen Corp., Carlsbad, CA, USA) and an Agilent 2100 Bioanalyzer (Agilent Technologies, Palo Alto, CA, USA), respectively. Libraries were prepared using a Truseq stranded mRNA kit (Illumina, San Diego, USA) following the manufacturer's instructions. Sequencing was conducted on an Illumina MiSeq sequencing system using the Illumina MiSeq reagent kit V3 (Illumina, San Diego, USA) following the manufacturer's instructions.

\section{Data analysis}

S. sclerotiorum transcripts available in the database (http:// www.broadinstitute.org/annotation/genome/sclerotinia_scle rotiorum/MultiHome.html) were used as a reference for mapping the short reads using CLC Genomics Workbench 7.0.4 (http://www.clcbio.com). Gene expression was estimated by extracting read counts as integers from the CLC Genomics alignments. The count data were normalized to generate effective library sizes using the scaling method Trimmed Means of Means values (TMM) [45]. Statistical analysis was performed with these data using a generalized linear model linked to the negative binomial distribution performed using the EdgeR package [45]. Pair-wise analyses were performed to assess differential gene expression using the control library as a common reference standard. Genes were considered differentially expressed where the probability after adjustment for multiple hypothesis testing [false discovery rate (FDR)] was less than 0.05 . The extent of the observed differential expression was considered meaningful if the fold change exceeded a factor of two. Finally, all significantly up-regulated genes at different sampling times were assigned a functional classification using the BLAST2GO plugin (v1.4.4) in the CLC Genomics Workbench 8.0.1 for functional annotation using Interpro and the NCBI refseq protein database. Gene ontology (GO) terms for each gene were extracted. The results were filtered to remove top-level annotations and apply the GO-slim categorization from Aspergillus in order to summarize the results. Blast2GO ran ANNEX [46] to add implicit GO terms for a more complete annotation. Finally, Blast2GO was used to calculate the abundance of GO classifications for the significantly up-regulated genes for each time point. Candidate genes were categorized into different groups based on known functions of orthologous genes in other fungi.

\section{Validation of RNA-Seq analysis using droplet digital PCR (ddPCR)}

cDNA was synthesized from $1 \mu \mathrm{g}$ of total RNA using the iScript Reverse Transcription Supermix for RT-qPCR kit (Bio-Rad, CA, USA) following the manufacturer's instructions. The ddPCR was conducted with three biological replicates using a droplet digital PCR QX200 system (Bio-Rad, CA, USA). No-reverse transcriptase (no-RT) controls were also used to detect genomic DNA contamination. Primers and probe for each gene were designed using PrimerQuest tool (IDT) and all probes were labeled with fluorescein amidite (FAM), except for the reference gene ( $\beta$-tubulin) which was labeled with hexachloro-fluorescein (HEX). Sequences and details of primers and probes have been provided in Additional file 1: Table S1. The ddPCR reaction mixtures $(20 \mu \mathrm{l})$ contained 1X ddPCR supermix (Bio-Rad Laboratories, Hercules, CA), $900 \mathrm{nM}$ of each primer, $250 \mathrm{nM}$ of probe and $4 \mu \mathrm{l}$ of 1:100 diluted cDNA. The PCR was performed in a C1000 Touch Thermal Cycler (Bio-Rad, CA, USA) with the following cycling conditions: $95^{\circ} \mathrm{C}$ for $10 \mathrm{~min} ; 50$ cycles of $94^{\circ} \mathrm{C}$ for $30 \mathrm{~s}, 53^{\circ} \mathrm{C}$ for $75 \mathrm{~s}$, Ramp $2^{\circ} \mathrm{C} / \mathrm{s} ; 98^{\circ} \mathrm{C}$ for $10 \mathrm{~min}$. The droplet generation and reading for ddPCR were conducted using a Droplet Generator and Reader (Bio-Rad QX200 system), respectively, according to the manufacturer's instructions. The gene expression ratio was calculated by QuantaSoft droplet reader software (Bio-Rad). The expression of the $\beta$-tubulin gene (SS1G_ 04652) is constant during the infection, confirming its validity as a reference gene. The fold change in the expression of each gene was calculated by dividing the ratio of the target to the reference ( $\beta$-tubulin) gene for each time point by the ratio from the sample collected from fungi grown in culture (i.e. time zero).

\section{Results and discussion}

\section{RNA sequencing}

Illumina sequencing (RNA-Seq) was used to conduct sequential transcriptional profiling in order to identify differentially expressed genes involved in S. sclerotiorum establishment on and subsequent infection of $B$. napus. Mycelia were collected from liquid media immediately prior to inoculation (time 0 ) and at $1,3,6,12,24$ and 48 hpi. The number of reads per each biological replicate per each time point is shown in Additional file 2: Table S2. A total of 40,210,134 paired-end reads were generated. Reads mapped to 14,503 of the 14,522 predicted genes (99\% of 
total reference transcripts), indicating sufficient sequencing depth. Genes with expression ratios greater than two relative to the inoculum grown on MS-Glu medium and a false discovery rate (FDR) $p$-value correction of $<0.05$ were considered to be up-regulated. The numbers of upregulated genes were 584, 582, 526, 371, 822 and 1283 at $1,3,6,12,24$ and $48 \mathrm{hpi}$, respectively, ranging from $2.6 \%$ to $8.8 \%$ of total expressed genes. The RNA-Seq data was submitted to NCBI (accession \# GSE83935) and the list of up-regulated genes with BLAST2GO annotation can be found in Additional file 3: Table S3.

To confirm the relatedness of the three biological replicates and the accuracy of the RNA-Seq analysis, principal component analysis (PCA) was conducted (Fig. 1). Individual replicates of each time point clustered together, indicating a high degree of similarity in the expression profiles and low biological variability among the experimental replicates. Of the early infection time points sampled (1-12 hpi), the $1 \mathrm{hpi}$ sample was most different from the zero time point with successive early time points becoming increasingly more similar to the inoculum. PCA also showed a clear distinction between the $S$. sclerotiorum transcriptomes at 24 and 48 hpi compared to the other time points which was due to a significant increase in both the number and types of genes expressed at these time points.

\section{Gene ontology analysis of up-regulated genes}

To obtain an overall view of the genes involved in $S$. sclerotiorum infection, gene ontology (GO) analysis of the up-regulated genes was performed. Blast2GO using different forms of annotations, including Interpro, GOslim, enzyme code and Annex, was used to calculate the abundance of GO classifications in each of these ontology categories, molecular function (Fig. 2) and biological processes (Fig. 3) for each time point. In total, 25\%, 25\%, $26 \%, 22 \%, 15 \%$ and $18 \%$ of the up-regulated genes at the $1,3,6,12,24$ and 48 hpi sampling times, respectively, were annotated as encoding proteins with unknown functions and therefore could not be assigned to a GO category. The majority of up-regulated genes in the molecular function group fell into the subcategories of oxidoreductase and hydrolase activity in all sampling times. The highest proportion of up-regulated genes belonging to oxidoreductase and hydrolase activity subcategories was at $24 \mathrm{hpi}$, and declined at $48 \mathrm{hpi}$ coincident with the appearance of visible necrotic lesions. For the other molecular function subcategories, genes classified as encoding proteins with transferase, transporter, protein binding, DNA binding, protein kinase and signal transducer activity, the lowest proportion of up-regulated genes was at $48 \mathrm{hpi}$, suggesting a decrease in the expression of these genes after the start of necrotic stage.

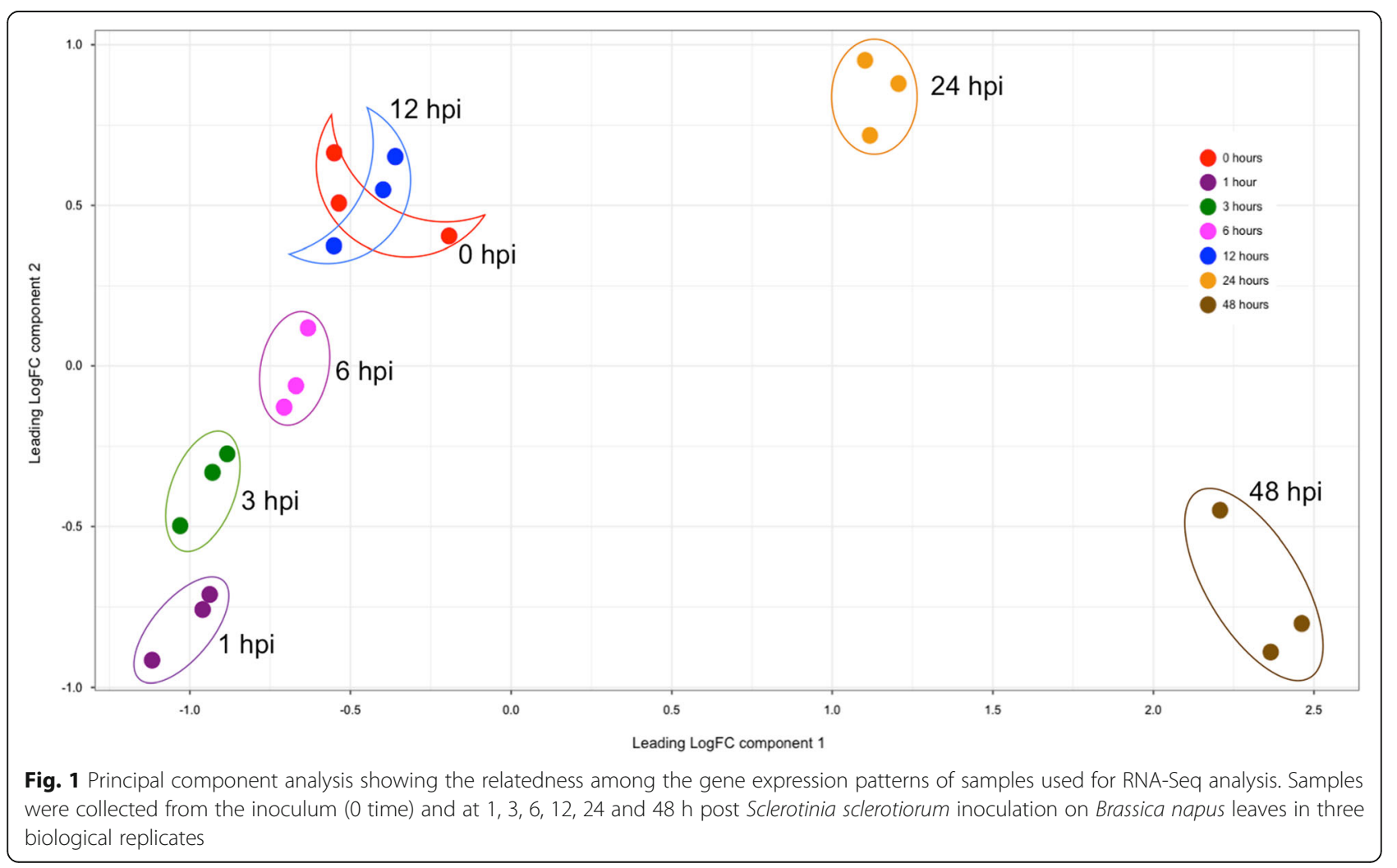




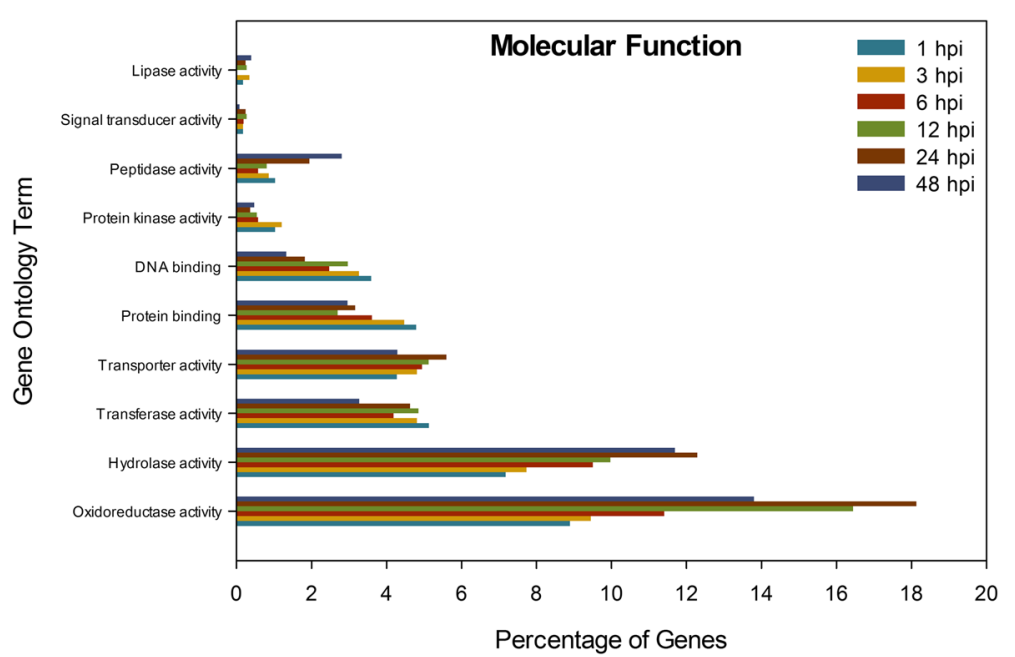

Fig. 2 Percentage of genes encoding enzymes assigned to Molecular Function subcategories (indicated) that were induced upon Sclerotinia sclerotiorum infection of Brassica napus. Gene ontology analysis was conducted using Blast2Go software. hpi, hours post-inoculation

The majority of up-regulated genes within the biological processes category belonged to carbohydrate metabolic process subcategory. The highest proportion of genes within this subcategory was found at $24 \mathrm{hpi}$, with a notable increase compared with $12 \mathrm{hpi}$, and a decline by $48 \mathrm{hpi}$, similar to the hydrolytic activity subcategory. This supports the connection between carbohydrate metabolic activity and hydrolytic enzyme activity as these processes work in concert to supply nutrients and energy for mycelial proliferation and to facilitate the transition to the necrotrophic phase occurring beyond 24 hpi in this experimental system.

The highest proportion of up-regulated genes related to transcription and signal transduction was observed at 1 and $3 \mathrm{hpi}$, respectively. This is expected as the pathogen needs to modulate the expression of a wide variety of developmental and metabolic genes during the early stages of the infection as it penetrates the host cuticle and then establishes within the host. Beyond this, the 6 hpi time point had the highest percentage of upregulated genes involved in response to stress. This is likely a response to the exposure of the pathogen to host plant defense mechanisms. Kabbage et al. [3] proposed that a brief biotrophic phase occurs soon after cuticle penetration where the pathogen either remains undetected or compromises/tolerates host defenses. The induction of stress-related genes soon after cuticle penetration would alleviate some of the effects of these stress conditions and allow host colonization. Interestingly, after events leading to cuticle penetration (1 hpi), dramatic changes in gene expression appear to subside as the overall gene expression profiles at 3, 6 and $12 \mathrm{hpi}$

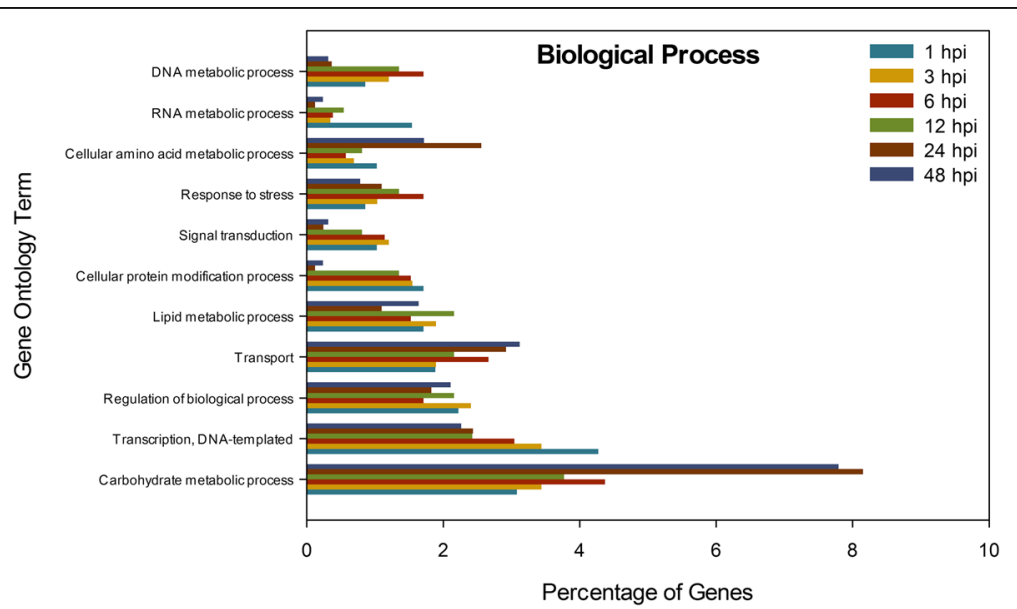

Fig. 3 Percentage of genes encoding enzymes assigned to Biological Process subcategories (indicated) that were induced upon Sclerotinia sclerotiorum infection of Brassica napus. Gene ontology analysis was conducted using Blast2Go software. hpi, hours post-inoculation 
become increasingly similar to that of the inoculum at time 0 (Fig. 1). This period may constitute the biotrophic phase which is followed by a mycelial ramification phase ( $24 \mathrm{hpi})$ and finally a necrotic phase (48 hpi), each of which have unique expression profiles. This is in accordance with the gene expression profiles of $B$. cinerea on Arabidopsis thaliana leaves where three distinct groups of genes were identified, these being early, outset of colonization and complete colonization, based on expression patterns [47].

\section{Validation of RNA-Seq analysis using droplet digital PCR (ddPCR)}

Three different types of genes from the RNA-Seq data list were selected for validation, including three highly expressed genes that were induced in most of the sampling times (SS1G_07027, SS1G_07661 and SS1G_08104 genes encoding a hypothetical protein, cutinase and acetylxylan esterase, respectively), three that were not induced during the sampling time points (SS1G_14133, SS1G_02486 and SS1G_05839 genes encoding SSITL, SsCaf1 and SsBi1, respectively) and four well-characterized $S$. sclerotiorum genes (SS1G_08218, SS1G_10796, SS1G_10167 and SS1G_07355 encoding oxaloacetate acetyl hydrolase $(\mathrm{OAH})$, oxalate decarboxylase, SsPG1 and the Pac1 transcription factor, respectively). The ddPCR analysis generated patterns of expression for the induced genes that were very similar to that predicted from the RNA-Seq data (Fig. 4). Only one out of the seven genes (SS1G_08104) tested showed a slightly different trend between ddPCR and RNA-Seq. Previous work showed about $90 \%$ correlation between $\mathrm{qPCR}$ and RNA-Seq [48] suggesting that slight variation between the two methods is expected, but is generally negligible. Furthermore, for the three genes that were not induced in the RNA-Seq analysis, the fold-change in expression did not exceed a factor of 2 when examined by ddPCR, providing additional evidence that they were not induced in the current study (data not shown).

\section{Functional classification of the genes}

To simplify the exploration of genes expressed during the course of S. sclerotiorum infection, genes were clustered based on expression patterns (Additional file 4: Figure S1) and categorized based on their functional annotation and predicted roles in pathogenicity or virulence; these are discussed below.

\section{Hydrolytic enzymes}

The current study revealed that a large number of the genes induced during infection encoded enzymes with hydrolytic activity (Table 1). The largest group of these genes encoded carbohydrate-active enzymes (CAZymes). Most of the CAZyme genes predicted in the S. sclerotiorum genome by Amselem et al. [38] were up-regulated during infection in this study and in the study by Lyu et al., [49] which examined different $S$. sclerotiorum developmental stages. These results consistently support the important role of these enzymes during infection. The majority of the genes encoding CAZymes reported in the current study were from the glycoside hydrolase $(\mathrm{GH})$ and carbohydrate esterase (CE) families. The expression of numerous genes encoding GH and CE enzymes in the current study and in a similar study of Sclerotinia homoeocarpa reflects the ability of these pathogens to infect a wide range of plant hosts [41]. These are discussed in more detail below.

1.1 Cutinases/lipases The first barrier to pathogen invasion is the plant cuticle, a composite layer of C:16 and C:18 fatty acids and their derivatives that form cutin and surface waxes [50]. Among the genes from the $\mathrm{CE}$ family, the gene encoding the cutinase, SsCuta (SS1G_07661), was up-regulated during the early stages of infection (from 1-24 hpi). The induction of SsCuta soon after contact with the leaf surface in the current study agrees with the previous report showing that it was induced upon contact of mycelia with hard surfaces [4] and supports the predicted role of this enzyme in degrading plant cuticle. Additionally, it is not surprising that expression of this gene declined after 24 hpi since host penetration has already been achieved by this time. In addition to $\mathrm{SsCuta}$, three other genes from the lipid degradation group (Table 1), SS1G_09557, SS1G_01953 and SS1G_11930, were also induced during the early stages of infection. The similarity between the expression patterns of these genes and that of SsCuta, as well as their potential lipolytic enzymatic activity, suggest that these enzymes may also be involved in host penetration. Evidence that lipase acts as virulence factor in fungal phytopathogens was observed in Botrytis cinerea (Lip1) [51]. The secreted lipase in Fusarium graminearum encoded by $F g L 1$ is also a virulence factor contributing to the infection of cereals [52]. Genes encoding other lipases and members of the CE family, such as the cutinases (SS1G_13386 and SS1G_12907) and an extracellular lipase (SS1G_14146), were significantly up-regulated at 24 and $48 \mathrm{hpi}$ (Table 1).

1.2 Plant cell wall degrading enzymes Once the cuticle has been breached, the pathogen must establish within the host and then proceed to ramify through host tissues. The production of enzymes that degrade plant cell wall components physically allows this to occur, while providing nutrients to drive the infection process [53]. The primary plant cell wall is composed mainly of cellulose, hemicellulose and pectin, along with structural glycoproteins (e.g. hydroxyproline-rich extensins) and phenolic esters (e.g. ferulic and coumaric acid). The secondary cell wall consists mostly of lignin, a highly cross-linked phenolic macromolecule. 

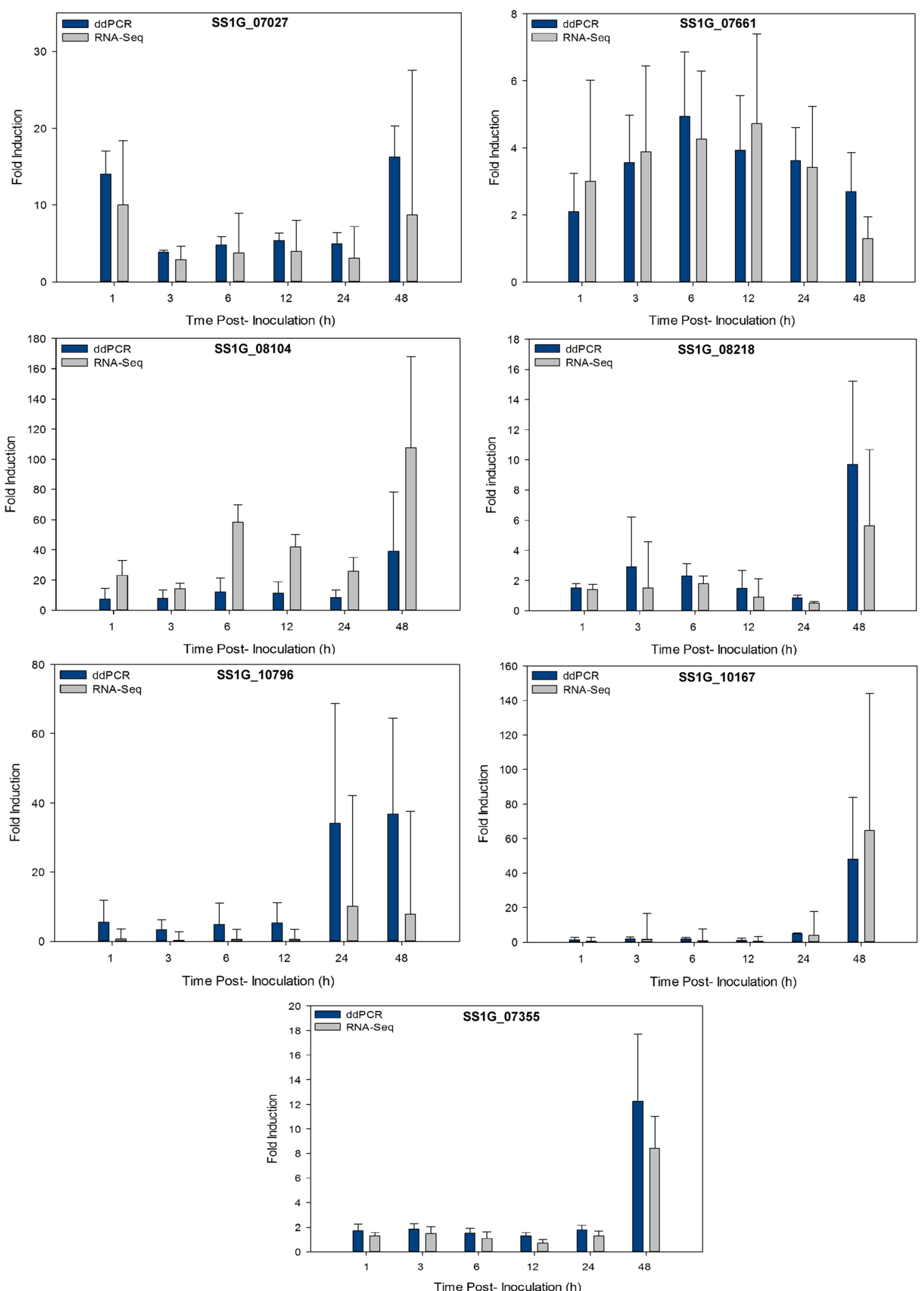

Fig. 4 Expression of various Sclerotinia sclerotiorum genes during infection of Brassica napus as determined by RNA-Seq and droplet digital PCR (ddPCR) analysis. Histograms show the relative expression level (fold change) and are reported as means and standard errors of three biological replicates for hour post-inoculation (hpi) 
Table 1 Description and expression of up-regulated genes encoding hydrolytic enzymes

\begin{tabular}{|c|c|c|c|c|c|c|c|}
\hline \multirow[t]{2}{*}{ Gene ID } & \multirow[t]{2}{*}{ Description $^{a}$} & \multicolumn{6}{|c|}{ Expression level (hpi) } \\
\hline & & 1 & 3 & 6 & 12 & 24 & 48 \\
\hline \multicolumn{8}{|l|}{ 1. Lipid Degradation } \\
\hline SS1G_07661 & cutinase (SsCuta) & 3 & 3.9 & 4.3 & 4.7 & 3.4 & - \\
\hline SS1G_13386 & cutinase & - & - & - & - & 14.9 & 29.6 \\
\hline SS1G_12907 & cutinase & - & - & - & - & 29.7 & 236 \\
\hline SS1G_09557 & lipase/esterase & 3.4 & 5.5 & 6.5 & 6.1 & 2.6 & - \\
\hline SS1G_11473 & lipase/esterase & 2.2 & - & - & - & - & 3.2 \\
\hline SS1G_05990 & lipase/esterase & - & - & - & - & 2.2 & - \\
\hline SS1G_00767 & lipase/esterase & - & - & - & - & - & 34.4 \\
\hline SS1G_03597 & lipase/esterase & - & - & - & - & - & 5.1 \\
\hline SS1G_01849 & lipase/esterase & - & - & - & - & - & 2.7 \\
\hline SS1G_08869 & lipase class 3 & - & - & - & - & - & 2.8 \\
\hline SS1G_14146 & extracellular lipase & - & - & - & - & 31.5 & 38.7 \\
\hline SS1G_04490 & extracellular lipase & - & - & - & - & 2.5 & 3 \\
\hline SS1G_00877 & extracellular lipase & - & - & - & - & - & 4.3 \\
\hline SS1G_14441 & triacylglycerol lipase & - & - & - & - & 5 & 6.9 \\
\hline SS1G_13982 & triacylglycerol lipase & - & - & - & - & 4.8 & 27.5 \\
\hline SS1G_01472 & triacylglycerol lipase & - & - & - & - & 3.6 & 13.1 \\
\hline SS1G_03007 & alpha beta-hydrolase (esterase) & - & 2.6 & 2.6 & - & - & - \\
\hline SS1G_09718 & alpha beta-hydrolase (esterase/lipase) & - & 2.1 & 2.9 & - & - & - \\
\hline SS1G_01703 & alpha beta-hydrolase (esterase/lipase) & - & 3.9 & 3.5 & 2.2 & 3.8 & 6.5 \\
\hline SS1G_13361 & alpha beta-hydrolase (esterase/lipase) & - & - & - & 2.3 & 3.2 & 2.2 \\
\hline SS1G_11402 & alpha beta hydrolase (esterase/lipase) & - & - & - & 3.6 & 4.8 & 3.7 \\
\hline SS1G_13263 & alpha beta hydrolase (esterase/lipase) & - & - & - & - & 3.8 & 3.8 \\
\hline SS1G_08133 & alpha beta-hydrolase (esterase/lipase) & - & - & - & - & - & 2.5 \\
\hline SS1G_02163 & alpha beta-hydrolase (esterase/lipase) & - & - & - & - & - & 12.5 \\
\hline SS1G_01953 & GDSL lipase acylhydrolase family protein & 3.6 & 4 & 5 & 4.9 & 4.8 & 5.6 \\
\hline SS1G_13560 & GDSL lipase acylhydrolase family protein & - & - & - & - & 2.7 & 2.7 \\
\hline SS1G_06389 & GDSL lipase acylhydrolase family protein & - & - & - & - & - & 2.7 \\
\hline SS1G_02708 & cellulose-binding GDSL lipase & - & - & - & - & 3.3 & 7.8 \\
\hline SS1G_14289 & cellulose-binding GDSL lipase & - & - & - & - & 5.3 & 6.6 \\
\hline SS1G_03610 & cellulose-binding GDSL lipase & - & - & - & - & 5.1 & 22.4 \\
\hline SS1G_04592 & cellulose-binding GDSL lipase & - & - & - & - & 11.8 & 9.4 \\
\hline SS1G_11930 & carboxylesterase/lipase (cholinesterase) & 4 & 3.9 & 8.9 & 5.2 & - & - \\
\hline SS1G_00376 & carboxylesterase/lipase (cholinesterase) & - & - & - & - & 4.4 & 9.2 \\
\hline SS1G_09613 & carboxylesterase/lipase (cholinesterase) & - & - & - & - & - & 4.7 \\
\hline SS1G_04422 & carboxylesterase/lipase (cholinesterase) & - & - & - & - & - & 3.2 \\
\hline SS1G_11853 & carboxylesterase/lipase (cholinesterase) & - & - & - & - & - & 3 \\
\hline SS1G_04030 & lysophospholipase & - & - & 3.6 & - & 2.3 & - \\
\hline SS1G_10482 & lysophospholipase & - & - & - & - & - & 4.1 \\
\hline \multicolumn{8}{|c|}{ 2. Polysaccharide Degradation } \\
\hline \multicolumn{8}{|l|}{2.1 Cellulose } \\
\hline SS1G_08493 & beta-1,4-endo-glucanase & 2.6 & - & - & - & - & - \\
\hline SS1G_00891 & beta-1,4-endo-glucanase (cellulase) & - & 4.5 & 5.8 & 3.5 & - & 3.8 \\
\hline SS1G_01485 & beta-glucanase & - & - & - & - & - & 2.1 \\
\hline SS1G_09365 & endo-glucanase & - & - & - & - & 2.7 & - \\
\hline
\end{tabular}


Table 1 Description and expression of up-regulated genes encoding hydrolytic enzymes (Continued)

\begin{tabular}{|c|c|c|c|c|c|c|c|}
\hline SS1G_03387 & endo-glucanase & - & - & - & - & 5.3 & 41.4 \\
\hline SS1G_08837 & endo-glucanase & - & - & - & - & 10.3 & 36.4 \\
\hline SS1G_04945 & endo-glucanase & - & - & - & - & - & 2.3 \\
\hline SS1G_01828 & endo-glucanase & - & - & - & - & - & 2.6 \\
\hline SS1G_09821 & endo-glucanase & - & - & - & - & - & 144 \\
\hline SS1G_03041 & endo-glucanase & - & - & - & - & - & 292 \\
\hline SS1G_00321 & endo-glucanase & - & - & - & - & - & 4.2 \\
\hline SS1G_00471 & endo-glucanase & - & - & - & - & - & 2.1 \\
\hline SS1G_06037 & exo-glucanase & - & 3 & 3.2 & - & 9.9 & 12.3 \\
\hline SS1G_02334 & exo-glucanase (cellobiohydrolyase) & - & - & - & - & 3.8 & - \\
\hline SS1G_09020 & exo-glucanase & - & - & - & - & 15.1 & 79 \\
\hline SS1G_00892 & exo-glucanase & - & - & - & - & 5.4 & 22.2 \\
\hline SS1G_09118 & exo-glucanase & - & - & - & - & - & 2.5 \\
\hline SS1G_02245 & exo-glucanase & - & - & - & - & - & 3.3 \\
\hline SS1G_13872 & exo-glucanase & - & - & - & - & - & 7.6 \\
\hline SS1G_02501 & Concanavalin A-like lectin/glucanase & 5.1 & 8.7 & 6.1 & 3.1 & - & - \\
\hline SS1G_08907 & glycoside hydrolase (beta-glucanase) & 2.6 & - & - & - & - & - \\
\hline SS1G_07863 & cellobiose dehydrogenase & 3.7 & - & - & - & - & 22.1 \\
\hline SS1G_05151 & cellobiose dehydrogenase & - & - & - & - & 5.9 & 6.6 \\
\hline SS1G_05118 & beta-glucosidase & 2.4 & - & - & - & - & - \\
\hline SS1G_01662 & beta-glucosidase & - & - & - & - & 2.8 & 9.5 \\
\hline SS1G_09366 & beta-glucosidase & - & - & - & - & 2.8 & 8 \\
\hline SS1G_06304 & beta-glucosidase & - & - & - & - & 2.6 & 6.8 \\
\hline SS1G_07847 & beta-glucosidase & - & - & - & - & 3.1 & 4.7 \\
\hline SS1G_07146 & beta-glucosidase & - & - & - & - & 7.4 & 18.7 \\
\hline SS1G_13255 & beta-glucosidase & - & - & - & - & 40.4 & 54.8 \\
\hline SS1G_05368 & beta-glucosidase & - & - & - & - & 3.2 & 4.6 \\
\hline SS1G_12622 & beta-glucosidase & - & - & - & - & - & 2.1 \\
\hline SS1G_07162 & beta-1,4-glucosidase & - & - & - & - & - & 2.3 \\
\hline SS1G_09129 & beta-glucosidase & - & - & - & - & - & 13.1 \\
\hline SS1G_04264 & beta-glucosidase & - & - & - & - & - & 2.2 \\
\hline SS1G_01021 & beta-glucosidase & - & - & - & - & - & 5.2 \\
\hline \multicolumn{8}{|l|}{ 2.2 Pectin } \\
\hline SS1G_10167 & endo-polygalacturonase (SSPG1) & - & - & - & - & 3.7 & 64.6 \\
\hline SS1G_10698 & endo-polygalacturonase (SSPG3) & - & - & - & - & - & 5.5 \\
\hline SS1G_05832 & exo-polygalacturonase & 4.1 & 6.5 & - & - & 4.2 & 32.4 \\
\hline SS1G_02553 & exo-polygalacturonase (exoPG2) & - & - & - & - & - & 22.6 \\
\hline SS1G_04207 & exo-polygalacturonase (exoPG1) & - & - & - & - & 14.6 & 168 \\
\hline SS1G_12057 & exo-polygalacturonase & - & - & - & - & - & 4.2 \\
\hline SS1G_03540 & pectin lyase & - & - & - & - & 4.5 & 3.3 \\
\hline SS1G_10071 & pectin lyase & - & - & - & - & - & 6.2 \\
\hline SS1G_14449 & pectin lyase & - & - & - & - & - & 2.4 \\
\hline SS1G_04551 & pectin methylesterase & - & - & - & - & 11.6 & 35.8 \\
\hline SS1G_00332 & pectin methylesterase & - & - & - & - & 33.9 & 178 \\
\hline SS1G_03286 & pectin methylesterase & - & - & - & - & 4.8 & 16.5 \\
\hline SS1G_00468 & pectin methylesterase & - & - & - & - & - & 21.4 \\
\hline
\end{tabular}


Table 1 Description and expression of up-regulated genes encoding hydrolytic enzymes (Continued)

\begin{tabular}{|c|c|c|c|c|c|c|c|}
\hline SS1G_04095 & rhamnogalacturanan acetylhydrolase & 2.8 & 6.2 & 7.7 & 5.3 & 2.7 & - \\
\hline SS1G_12048 & rhamnogalacturanan acetylhydrolase & - & - & - & - & - & 33.5 \\
\hline SS1G_11992 & rhamnogalacturonan acetylesterase & - & - & - & - & - & 37.5 \\
\hline SS1G_09857 & rhamnogalacturonyl hydrolase & - & - & - & - & - & 8.6 \\
\hline SS1G_12964 & alpha-I-rhamnosidase & - & - & 17.5 & - & - & - \\
\hline SS1G_13501 & alpha-I-rhamnosidase & - & - & - & - & 3.1 & 21.2 \\
\hline SS1G_04541 & alpha-I-rhamnosidase & - & - & - & - & 2 & 8.3 \\
\hline SS1G_08229 & rhamnogalacturonase & 2.1 & 4.5 & 4.5 & 2.6 & - & - \\
\hline SS1G_07039 & rhamnogalacturonase & - & - & - & - & - & 6 \\
\hline SS1G_04552 & endo-xylogalacturonan hydrolase & - & - & - & - & 7.1 & 15.9 \\
\hline \multicolumn{8}{|l|}{2.3 Hemicellulose } \\
\hline SS1G_12191 & endo-1,4-beta-xylanase & - & 5.3 & 7.9 & 4.6 & 6.4 & 25 \\
\hline SS1G_10092 & endo-beta-xylanase & - & - & - & - & - & 155 \\
\hline SS1G_03618 & endo-beta-xylanase & - & - & - & - & - & 451 \\
\hline SS1G_07749 & endo-beta-xylanase & - & - & - & - & - & 56.7 \\
\hline SS1G_05140 & xylanase & 3.6 & 3.5 & 4.2 & 4.3 & 3.4 & 8.1 \\
\hline SS1G_08104 & acetylxylan esterase & 23 & 14.2 & 58.3 & 42.1 & 26 & 107 \\
\hline SS1G_05434 & acety|xylan esterase & - & - & - & - & 4 & 11.8 \\
\hline SS1G_00746 & beta-mannosidase & - & - & - & - & 6.6 & 42.3 \\
\hline SS1G_05977 & beta-mannosidase & - & - & - & - & 3.6 & 6.4 \\
\hline SS1G_08208 & endo-1,4-beta-mannosidase & - & - & - & - & 3.6 & 8.9 \\
\hline SS1G_08118 & alpha-xylosidase & - & - & - & - & 4.5 & 3.8 \\
\hline SS1G_09367 & alpha-xylosidase & - & - & - & - & 2.2 & - \\
\hline SS1G_11535 & alpha-fucosidase & - & - & - & - & - & 2.5 \\
\hline SS1G_04662 & alpha-galactosidase & - & - & - & - & 4.5 & 3.9 \\
\hline SS1G_03386 & alpha-galactosidase & - & - & - & - & - & 3.7 \\
\hline SS1G_07904 & feruloyl esterase & - & - & - & - & 3.6 & 12 \\
\hline SS1G_02462 & alpha-l-arabinofuranosidase & - & - & - & - & 5.3 & 21 \\
\hline SS1G_03602 & alpha-l-arabinofuranosidase & - & - & - & - & 6.3 & 17.6 \\
\hline \multicolumn{8}{|c|}{ 2.4 Arabinogalactans } \\
\hline SS1G_01216 & arabinogalactan endo-beta-galactosidase & 4.1 & 9.6 & 11.9 & 7.2 & 4.1 & - \\
\hline SS1G_11585 & arabinogalactan endo-beta-galactosidase & - & - & - & - & - & 19.3 \\
\hline SS1G_02618 & galactan 1,3-beta-galactosidase & - & - & - & - & 5.7 & 11 \\
\hline SS1G_10842 & beta-galactosidase & 4.1 & 2.5 & 2.4 & - & 2.2 & 5.8 \\
\hline SS1G_11763 & beta-galactosidase & - & - & - & - & 4.7 & - \\
\hline SS1G_01572 & beta-galactosidase & - & - & - & - & 6.8 & 18.3 \\
\hline SS1G_03647 & beta-galactosidase & - & - & - & - & 3.5 & 6.9 \\
\hline SS1G_02781 & beta-galactosidase & - & - & - & - & - & 2.7 \\
\hline SS1G_09866 & 1,6-beta-galactanase & - & 4.8 & 4.5 & 3.1 & 3 & 12.4 \\
\hline SS1G_11922 & arabinan endo-1,5-alpha-L-arabinosidase & - & - & - & - & 144.3 & 245 \\
\hline SS1G_01238 & beta-D-glucuronidase & 7.6 & - & 4.7 & - & - & - \\
\hline SS1G_02620 & beta-glucuronidase & - & - & - & - & 3.5 & 8.2 \\
\hline \multicolumn{8}{|l|}{2.5 Lignin } \\
\hline SS1G_04196 & dihydrogeodin oxidase/laccase & - & 3.1 & 2.6 & 4.6 & - & - \\
\hline SS1G_06365 & dihydrogeodin oxidase/laccase & - & 3.1 & 5.7 & 4.1 & - & 3.4 \\
\hline SS1G_05112 & dihydrogeodin oxidase/laccase & - & - & - & - & - & 6 \\
\hline
\end{tabular}


Table 1 Description and expression of up-regulated genes encoding hydrolytic enzymes (Continued)

\begin{tabular}{|c|c|c|c|c|c|c|c|}
\hline \multicolumn{8}{|l|}{ 2.6 Starch } \\
\hline SS1G_01776 & alpha-amylase & - & - & 2.1 & - & - & - \\
\hline SS1G_11100 & alpha-amylase & - & - & - & - & 6.6 & 5.2 \\
\hline SS1G_01083 & alpha-glucosidase & 2.4 & 4.7 & 3 & - & 4.6 & 5.3 \\
\hline SS1G_01005 & alpha-glucosidase & - & - & - & - & 9.2 & 7.2 \\
\hline \multicolumn{8}{|l|}{ 2.7 Mannans } \\
\hline SS1G_10867 & endo-1,6-alpha-mannosidase & - & - & - & - & 6.4 & - \\
\hline SS1G_04468 & endo-1,6-alpha-mannosidase & - & - & - & - & 7.5 & 25.3 \\
\hline SS1G_12937 & endo-1,6-alpha-mannosidase & - & - & - & - & - & 10.4 \\
\hline SS1G_05110 & endo-1,6-alpha-mannosidase & - & - & - & - & - & 3.5 \\
\hline SS1G_11579 & endo-1,6-alpha-mannosidase & - & - & - & - & - & 3.5 \\
\hline SS1G_09229 & alpha 1,2 mannosidase & - & - & - & - & 55 & 40.2 \\
\hline SS1G_00505 & alpha-1,2-mannosidase & - & - & - & - & 9.8 & 4.7 \\
\hline SS1G_04148 & alpha-mannosidase & - & - & - & - & 4.1 & 4 \\
\hline SS1G_02022 & alpha-mannosidase & - & - & - & - & 12.5 & 10.9 \\
\hline SS1G_04200 & alpha-mannosidase & - & - & - & - & 6 & 56.3 \\
\hline SS1G_01334 & alpha-mannosidase & - & - & - & - & - & 11.5 \\
\hline \multicolumn{8}{|l|}{2.8 Callose } \\
\hline SS1G_01422 & 1,3 (4)-beta-D-glucanase & 2.2 & - & - & - & - & - \\
\hline SS1G_10048 & 1,3 (4)-beta-D-glucanase & - & 2.1 & - & - & 2.1 & - \\
\hline \multicolumn{8}{|c|}{ 3. Protein Degradation } \\
\hline \multicolumn{8}{|l|}{ 3.1 Proteases } \\
\hline SS1G_10992 & caspase domain-containing protease & 2.1 & - & - & - & - & 7.1 \\
\hline SS1G_00862 & cysteine protease (calpain family) & 6.1 & 5.1 & 6.3 & 3.4 & 3.2 & - \\
\hline SS1G_09978 & peptidase (family 41 protein) & 3.2 & - & 3.2 & 2.9 & - & 6.4 \\
\hline SS1G_07836 & (acid) non-aspartyl protease (ACP1) & - & - & - & - & - & 40.4 \\
\hline SS1G_05329 & aspartyl protease & 12.2 & - & - & - & - & 4.6 \\
\hline SS1G_02870 & aspartyl protease & 2 & 2.6 & 3 & 3 & 2.7 & 3.3 \\
\hline SS1G_03181 & aspartyl protease & - & - & - & - & 3.9 & 16.6 \\
\hline SS1G_06534 & serine protease (trypsin-like) & 2.8 & 3 & - & - & - & 3.9 \\
\hline SS1G_12419 & serine protease (subtilisin-like) & - & - & 3 & - & - & - \\
\hline SS1G_07655 & serine protease (subtilisin-like) & - & - & - & - & 8.5 & 19.8 \\
\hline SS1G_02423 & serine protease (subtilisin-like) & - & - & - & - & - & 2.2 \\
\hline SS1G_03282 & serine protease (subtilisin-like) & - & - & - & - & - & 2.2 \\
\hline SS1G_07168 & serine protease (subtilisin-like) & - & - & - & - & - & 2.5 \\
\hline SS1G_12210 & serine protease (subtilisin-like) & - & - & - & - & - & 7.3 \\
\hline SS1G_05348 & metalloprotease & - & - & - & - & - & 2.6 \\
\hline SS1G_05349 & metalloprotease & - & - & - & - & - & 3.1 \\
\hline \multicolumn{8}{|l|}{3.2 Peptidases } \\
\hline SS1G_04565 & cytosolic no-pecific dipeptidase & - & - & - & - & 26 & 9.2 \\
\hline SS1G_10529 & cytosolic no-pecific dipeptidase & - & - & - & - & - & 2.2 \\
\hline SS1G_04140 & dipeptidyl-peptidase & - & 2.5 & - & - & - & - \\
\hline SS1G_03087 & membrane dipeptidase & - & - & - & - & - & 2.6 \\
\hline SS1G_03392 & proline dipeptidase & - & - & - & - & 4.5 & - \\
\hline SS1G_08920 & proline dipeptidase & - & - & - & - & 2.4 & 2 \\
\hline SS1G_04958 & tripeptidyl-peptidase & - & - & - & - & 5 & 22.9 \\
\hline
\end{tabular}


Table 1 Description and expression of up-regulated genes encoding hydrolytic enzymes (Continued)

\begin{tabular}{|c|c|c|c|c|c|c|c|}
\hline SS1G_07268 & tripeptidyl-peptidase & - & - & - & - & 3.5 & 2.1 \\
\hline SS1G_13922 & tripeptidyl peptidase & - & - & - & - & 8.9 & 50.4 \\
\hline SS1G_09225 & tripeptidyl peptidase & - & - & - & - & 6.9 & 16.9 \\
\hline SS1G_09268 & tripeptidyl-peptidase & - & - & - & - & 5.9 & 3.4 \\
\hline SS1G_02857 & tripeptidyl peptidase & - & - & - & - & 6.4 & 2.5 \\
\hline SS1G_03518 & tripeptidyl peptidase & - & - & - & - & - & 6.1 \\
\hline SS1G_01236 & tripeptidyl peptidase & - & - & - & - & - & 9.7 \\
\hline SS1G_08558 & prolyl aminopeptidase & 2.5 & 4.3 & 4.4 & 3.4 & 2.4 & 2.2 \\
\hline SS1G_00617 & prolyl aminopeptidase & - & - & - & 9.5 & 4.9 & 5.2 \\
\hline SS1G_12775 & prolyl aminopeptidase & - & - & - & - & - & 3 \\
\hline SS1G_05449 & carboxypeptidase & - & 2.5 & - & - & 3.3 & 13.3 \\
\hline SS1G_12413 & carboxypeptidase & - & - & - & - & 3.5 & 4.2 \\
\hline SS1G_08855 & carboxypeptidase & - & - & - & - & 3.7 & 4.1 \\
\hline SS1G_03361 & carboxypeptidase & - & - & - & - & 4.2 & 21.1 \\
\hline SS1G_09475 & carboxypeptidase & - & - & - & - & - & 13.1 \\
\hline SS1G_13633 & carboxypeptidase & - & - & - & - & - & 7.6 \\
\hline SS1G_12499 & carboxypeptidase & - & - & - & - & - & 11.5 \\
\hline SS1G_04819 & carboxypeptidase & - & - & - & - & - & 5.5 \\
\hline \multicolumn{8}{|c|}{ 4. Other Hydrolytic Enzymes } \\
\hline SS1G_01113 & metallo-dependent amidohydrolase & - & - & - & - & 3.2 & - \\
\hline SS1G_09143 & metallo-dependent amidohydrolase & - & - & - & - & - & 6.1 \\
\hline SS1G_02141 & alpha beta-hydrolase & 4.6 & - & - & - & - & - \\
\hline SS1G_11096 & alpha beta-hydrolase & - & - & - & 2.1 & 5.7 & 2.6 \\
\hline SS1G_08093 & alpha beta-hydrolase (epoxide hydrolase) & - & - & - & - & 4.4 & 9.2 \\
\hline SS1G_04475 & endo-alpha-1,4-polygalactosaminidase & 3 & - & - & - & - & 3.8 \\
\hline SS1G_11842 & sialidase & 3.1 & - & 3.6 & - & - & 9.8 \\
\hline SS1G_01389 & polysaccharide lyase family 7 protein & - & 4.9 & 4.9 & 5.2 & - & - \\
\hline SS1G_01493 & glycoside hydrolase family 3 protein & - & - & - & - & 4.2 & 17 \\
\hline SS1G_09000 & glycoside hydrolase family 5 protein & 2.7 & - & - & - & 2.7 & - \\
\hline SS1G_02369 & glycoside hydrolase family 12 protein & - & - & - & - & - & 3.4 \\
\hline SS1G_04497 & glycoside hydrolase family 16 protein & - & - & - & - & - & 2 \\
\hline SS1G_09789 & glycoside hydrolase family 16 protein & - & - & - & - & - & 2.8 \\
\hline SS1G_06426 & glycoside hydrolase family 43 protein & - & - & - & - & 2.4 & 5.9 \\
\hline SS1G_07515 & glycoside hydrolase family 43 protein & - & - & - & - & 138 & 518 \\
\hline SS1G_07656 & glycoside hydrolase family 61 protein & - & - & - & - & - & 93.6 \\
\hline SS1G_09251 & glycoside hydrolase family 61 protein & - & - & - & - & - & 9.1 \\
\hline SS1G_12106 & glycoside hydrolase family 76 protein & - & - & - & - & 3 & 13 \\
\hline SS1G_12083 & glycoside hydrolase family 115 protein & - & - & - & - & 7.8 & 17 \\
\hline SS1G_04152 & glycoside hydrolase family 125 protein & - & 2.2 & - & - & 6 & 12.5 \\
\hline SS1G_12917 & glycoside hydrolase family 128 protein & - & - & - & - & 2.3 & 17.3 \\
\hline
\end{tabular}

${ }^{a}$ Annotation based on the presence of conserved PFAM domains and BLAST reports

${ }^{\mathrm{b}}$ Fold change relative to $0 \mathrm{~h}$ post inoculation (hpi). (-) No significant change in expression

More information about the genes can be found in Additional file 3: Table S3

The GH28 subfamily contains the polygalacturonases (PGs), enzymes that degrade cell wall pectin. Previously, four genes encoding endo-PGs (SsPG1, SsPG3, SsPG5, and $S s P G 6)$ and two genes encoding exo-PGs (SsXPG1 and SsXPG2) were found to be expressed during S. sclerotiorum infection of B. napus [23]. In the current study, the genes encoding SsPG1 (SS1G_10167) and SsPG3 (SS1G_10698) were up-regulated at 24-48 hpi and 48 
hpi with expression levels 3.7- 64.6 and 5.5-fold greater than the inoculum, respectively. The two exo-PGs genes, SsXPG1 (SS1G_04207) and SsXPG2 (SS1G_02553), were up-regulated at 24-48 hpi and 48 hpi with expression levels 14.6-168.3 and 22.6- fold greater than the inoculum, respectively. While the main pectin backbone is a homopolymer of $\alpha$ - $(1 \rightarrow 4)$-linked D-galacturonic acid residues, branched and unbranched side chains are appended to it that contain several different types of sugars. Most of the genes encoding these ancillary pectindegrading enzymes were highly expressed at 24 and 48 hpi in concert with the endo-PGs genes SsPG1 and SsPG3, except for SS1G_05832 (exo-PG), SS1G_04095 (rhamnogalacturanan acetylhydrolase) and SS1G_08229 (rhamnogalacturonase) which were up-regulated at 1-3 hpi, 1-24 hpi and 1-12 hpi, respectively.

Li et al. [23] reported that SsPG1 expression could be induced by contact with hard surfaces, while Bashi et al. [4] reported that SsPG1, but not SsPG2, was moderately induced by contact with B. napus leaves and that SsPG1 expression was restricted to the expanding margin of the lesion. They suggested that since SsPG1 expression was also induced by carbon starvation and repressed by galacturonic acid that it may be involved in both early penetration events and lesion expansion. During Phaseolus vulgaris infection, SsPG1 is induced during the later stages of the interaction (48-72 hpi), SsPG3 is up-regulated earlier at $12 \mathrm{hpi}$, while SsPG6 exhibits a bimodal pattern with peaks of expression at 6 and 48 hpi [40]. SsPG3 and SsPG6 are also potent inducers of light-dependent necrotic reactions [54]. Similarly, $B$. cinerea BcPG1 and BcPG2 exhibit strong necrosis-inducing activity [55] and deletion of either gene reduces $B$. cinerea virulence $[55,56]$. The induction of SsPG or orthologous genes well after host penetration and their ability to cause tissue necrosis suggests that the primary role of these enzymes is in lesion expansion and movement of the pathogen through the host tissues.

Many of the up-regulated GH and CE family members reported in this study also have a putative role in the degradation of hemicellulose and cellulose. This was similar to the previous results obtained for up-regulated GH genes in S. homoeocarpa [41]. Cellulose is a homopolymer of beta- $(1,4)$-linked D-glucose and is sequentially hydrolyzed into its component glucose by enzymes including cellulases (endo-1,4-glucanases), cellobiosidases (exo-glucanases) and beta-glucosidases. Numerous genes encoding putative cellulases were up-regulated at some point during the infection, mostly at the later stages, with SS1G_09821 and SS1G_03041, up-regulated 144 and 292 fold at $48 \mathrm{hpi}$ (Table 1). Genes encoding putative exoglucanases and beta-glucosidases followed a similar pattern of expression with most being up-regulated at the later stages and only a few during the earlier stages of the infection. In higher plants, hemicellulose comprises approximately $20 \%$ of the total biomass. Unlike the more homogenous cellulose, hemicellulose is composed not only of glucose, but of other sugars such as xylose, mannose, galactose, rhamnose, and arabinose. As such, its deconstruction requires a more complicated bevy of enzymatic reactions. Similar to the genes encoding cellulose-degrading enzymes, genes encoding putative hemicellulose degrading enzymes were also up-regulated later in the infection. The exceptions were genes encoding enzymes involved in the release of xylose from xylan (beta-1,4-linked xylose), namely SS1G_12191, SS1G_05140 and SS1G_08104, which were first induced at the earlier stages. Two other genes encoding xylanases (SS1G_10092 and SS1G_03618) were among the most highly induced genes found in this study with levels of expression 155 and 451 fold higher than that of the inoculum at $48 \mathrm{hpi}$. These patterns may attest to the abundance of this sugar in the plant cell wall and/or its significance to $S$. sclerotiorum nutrition. Interestingly, SS1G_10092 is likely orthologous to the gene encoding B. cinerea xylanase $11 \mathrm{~A}$ (90\% amino acid identity) which induces a strong necrotic reaction and is required for virulence [57]. Both proteins share a 30 amino acid region associated with necrotizing activity [57]. In the S. sclerotiorum - P. vulgaris interaction, SS1G_01493 (betaxylosidase) was up-regulated during the early stages before the emergence of visible necrotic symptoms on the stem, whereas genes encoding cellulose-degrading enzymes, SS1G_13255 (beta-1,4-glucanase) and SS1G_07146 (cellobiohydrolase), were induced during the later stages of infection coinciding with the formation of visible stem lesions [40]. In B. cinerea, the expression patterns of genes encoding xyloglucan-degrading enzymes was found to be vastly different dependent upon the host plant [58].

Two genes encoding other hemicellulose-degrading enzymes with alpha-L-arabinofuranosidase activity, SS1G_02462 and SS1G_03602, were also up-regulated at $24-48 \mathrm{hpi}$. These enzymes target the L-arabinofuranose residues of hemicellulose with pectin side chains [59]. Alpha-L-arabinofuranosidase was first reported in $S$. sclerotiorum by Yajima and Kav [42]. An earlier study on Sclerotinia fructigena suggested that there was significant correlation between the quantity of alpha-Larabinofuranosidase and virulence of this fungus through its contribution to disease initiation or fungal proliferation [60].

Arabinogalactans are structurally complicated branched galactans with arabinose side chains and can be found as either beta-1,4-galactans linked to rhamnogalacturonan I in pectin, or as beta-1,4-galactans associated with proteins [61]. As with the other cell wall degrading enzymes, the majority of the genes encoding putative arabinogalactandegrading enzymes were induced later in the infection 
(24-48 hpi) (Table 1). However, a gene encoding an arabinogalactan endo-beta-galactosidase (SS1G_01216) and another encoding a beta-galactosidase (SS1G_10842) were already induced at $1 \mathrm{hpi}$.

Mannans are polymers of mannose. Those with $\beta$ (1-4) linkages are typical of plant storage polysaccharides, while mannans with $\alpha$ (1-6) linked backbone and $\alpha(1-2)$ and $\alpha(1-3)$ linked branches are often associated with glycoproteins. As noted above, glycoproteins are a significant cell wall component and several genes encoding mannosidases capable of hydrolyzing these chemical bonds were up-regulated during the later stages of the infection (Table 1).

Lignin provides additional structure and rigidity to the plant cell wall and increased lignification is often a consequence of imposed biotic and abiotic stresses [62]. Three genes encoding extracellular dihydrogeodin oxidases were up-regulated at the mid to later stages of the infection (Table 1). All contain three multicopper oxidase domains which are often associated with enzymes, such as laccases, that oxidize phenolic compounds. Laccases are involved in the disassembly of lignin [63], though some may detoxify phenolic secondary metabolites as discussed below.

Collectively, the plethora of CAZymes expressed by Sclerotinia species allow this group of pathogens to break down most host polysaccharides to efficiently access nutrients from a wide variety of hosts. The expression patterns of the CAZyme genes in the current study, which were mostly expressed at later stages of infection (24-48 hpi), support the hypothesis that they are primarily involved in tissue maceration. Factors such as host plant species, the type of tissues being colonized and the environment also influence the expression of different CAZyme genes. In $B$. cinerea, the expression of PG genes, in particular BcPG1 and BcPG2, was markedly different on different hosts or on the same host at different temperatures [58]. This same phenomenon was observed with genes encoding various pectin lyases, pectate lyases and pectin methylesterases [58]. The large number of genes encoding CAZymes and their different patterns of expression strongly indicate that they make an important contribution to pathogenesis and host range through adaptation to various environmental and host factors.

1.3 Proteases Although CAZymes have captured most of the attention as the main group of hydrolytic enzymes involved in pathogenesis, there are other groups of hydrolytic enzymes which also play crucial roles. Many genes encoding endo-proteases, as well as mono-, diand tri-peptidyl peptidases were up-regulated during infection (Table 1). The in planta expression of the acp1 (non-aspartyl acid protease, SS1G_07836) was recorded during S. sclerotiorum infection of sunflower cotyledons with the peak expression level at 24-56 hpi [21]. Acp1 was also up-regulated in the current study with 40 -fold greater expression than the inoculum at $48 \mathrm{hpi}$. The expression of this gene only at the necrotic stage is in support of the previous study. During infection of $P$. vulgaris, acp 1 is first induced during the very early stages of the infection and then again at the later stages [40]. The expression of $a c p 1$ is regulated by several environmental factors including glucose and nitrogen starvation and acidification. The $\mathrm{PacC}$ transcription factor is involved in the regulation of acp1 expression under acidic conditions [21].

A gene encoding an aspartyl protease (aspS, SS1G_03629) from S. sclerotiorum was previously reported as being induced at the very early stages of the infection and was involved in decomposition of host defense proteins [22]. An aspartyl protease was also reported as a cell death-inducing factor secreted by S. sclerotiorum and B. cinerea [64]. Although the aspS gene was not up-regulated in the current study, genes encoding proteases similar to aspS (SS1G_05329 and SS1G_02870), were up-regulated at the very early stages of the infection in support of the previous studies. However, a gene encoding another aspartyl protease, SS1G_03181, was also detected in the $S$. sclerotiorum- $P$. vulgaris interaction with increased expression at the spreading necrosis stage [40]. SS1G_03181 was up-regulated at 24-48 hpi in the current study which is in agreement with these earlier findings.

Most of the genes encoding subtilisin-like serine proteases (SS1G_07655, SS1G_02423, SS1G_03282, SS1G_12210 and SS1G_07168) were up-regulated at the later stages of the infection (24-48 hpi) (Table 1). Subtilisins were proposed to play a role in penetration and colonization because of their ability to degrade plant cell wall glycoproteins or pathogenesis-related proteins [65]; however, the expression profiles of genes encoding these enzymes in the current study suggest that they are also involved in events occurring at the necrotic stage. Genes encoding enzymes with metalloprotease activities (SS1G_05348 and SS1G_05349) were also up-regulated at 48 hpi. These proteases play a role in degrading plant materials for nitrogen utilization [66]. In accordance with this notion, the majority of the genes encoding exo-peptidases, which complete the hydrolysis of peptides generated by endo-proteases into their component amino acids, were expressed at the later stages of the infection similar to the genes encoding serine and metalloproteases (Table 1).

The current study revealed one calpain family cysteine protease gene (SS1G_00862) that was induced at $1 \mathrm{hpi}$ and continued to be expressed during the middle stages of the infection out to $24 \mathrm{hpi}$. A caspase domain-containing cysteine protease (SS1G_10992) was also up-regulated at 1 hpi and was detected again at $48 \mathrm{hpi}$ at a higher expression level (7.1-fold greater than the inoculum). According to 
previous studies, these types of endo-peptidases contribute to programmed cell death (PCD) processes (reviewed by [67]). Among these, cysteine proteases, specifically the caspases, have a key role in PCD, more commonly apoptosis (reviewed by [67]). In addition, cysteine proteases along with other proteolytic systems such as calpain, $26 \mathrm{~S}$ proteasome, granzyme B, cathepsin D and matrix metalloproteinases also have a role during PCD processes [68]. There is a report suggesting that victorin, a toxin produced by Cochliobolus victoriae, induces proteolytic cleavage of the Rubisco large subunit (LSU) through activation of a host cysteine protease [69]. Considering these previous studies, understanding the precise role of these genes in the pathogenesis of S. sclerotiorum warrants further investigation as they may be involved in processes linked to phase transitions during the infection [11].

Proteases are important for S. sclerotiorum nutrition as protein forms about $10 \%$ of host cell protoplasm [70]. Numerous proteases were also captured in the $S$. homoeocarpa transcriptome, but these were mostly serine endo-proteases [41]. In the current study, the up-regulated endo-protease genes encoded enzymes with a much broader range of catalytic mechanisms. Several genes encoding putative aspartyl (active at acidic $\mathrm{pH}$ ) and cysteine (active at acidic to neutral $\mathrm{pH}$ ) proteases were up-regulated very early in the infection, while most of the serine proteases (active at high $\mathrm{pH}$ ) and two genes encoding metalloproteases were up-regulated at the later stages. It is possible that the coordinated and systematic deployment of proteolytic enzymes with different catalytic mechanisms reflects the lesion environment, the pathogen's nutritional requirements and interactions with host defense systems at different stages of the infection.

\section{Secondary metabolite biosynthesis}

Several genes encoding enzymes involved in the biosynthesis of secondary metabolites were up-regulated in the current study (Table 2). These included key enzymes associated with pathways for production of toxic compounds, including polyketide synthase (PKS), nonribosomal peptide synthase (NRPS), hybrid PKS/NRPS, and chalcone synthase (CHS). These fungal toxins interfere with host cell functions to suppress plant defense and/or enhance development of disease symptoms [71]. Of the various types of phytotoxic metabolites produced by $B$. cinerea, botrydial has been most intensively studied [72]. BcBOT1 is part of the botrydial biosynthesis pathway and encodes a cytochrome P450 monooxygenase [73]. Cytochrome P450 enzymes are also involved in the aflatoxin biosynthetic pathways [74]. SS1G_09638 and SS1G_11247 have some similarity to StcL gene, which is involved in aflatoxin biosynthesis in Aspergillus nidulans. These genes were expressed, but not up-regulated compared to the inoculum in the current study, while SS1G_13923 was up-regulated at 1 to 6 hpi (Table 3). It should be noted that while cytochrome P450 enzymes are involved in the production of secondary metabolites and mycotoxins, some members are also involved in the detoxification of host metabolites in different fungi [75].

Polyketides are a structurally diverse group of secondary metabolites derived from the decarboxylative condensation of malonyl-CoA and include many mycotoxins. In $B$. cinerea, two genes encoding PKS, BcPKS6 and BcPKS9, that act in concert to synthesize the phytotoxin botcinic acid, are up-regulated during infection of tomato leaves [76]. Transcripts from the S. sclerotiorum ortholog of PKS6 (SS1G_09237) were detected at $48 \mathrm{hpi}$, while PKS9 (SS1G_09240) was expressed, but not up-regulated in this study. This agrees with the findings of Pedras and Ahiahonu [77] regarding the absence of botcinic acid in S. sclerotiorum as both enzymes are required for its synthesis. Both botrydial and botcinic acid toxins contribute to the virulence of B. cinerea [76]. SS1G_02592 encodes a protein similar to ToxD which is involved in the synthesis of the polyketide lovastatin in Aspergillus terreus [78]. It was initially up-regulated within $1 \mathrm{hpi}$ with expression peaking at $48 \mathrm{hpi}$.

Among the genes similarly induced during the infection by B. cinerea and S. sclerotiorum are those conserved in ascomycetes and involved in the biosynthesis of melanin (PKS13), coprogen (NRPS6) and intracellular siderophores (NRPS2, NRPS3) [38]. PKS13 (SS1G_13322) and NRPS6 (SS1G_04250) were expressed, but not up-regulated in the current study, while homologues of the genes responsible for siderophore biosynthesis NRPS2 and NRPS3 (SS1G_03693T0 and SS1G_06185T0) were upregulated at 3-48 and 1-24 hpi, respectively. Siderophores scavenge iron and are important virulence factors for many pathogens, including phytopathogenic fungi such as Cochliobolus heterostrophus [79] and Aspergillus fumigatus [80]. Iron-containing cofactors, such as heme and siroheme are required for the catalytic activity of enzymes associated with nitrogen and sulfur assimilation, as well as xenobiotic detoxification [81]. In the current study, a gene encoding siroheme synthase (SS1G_09177), also known as S-adenosyl-L-methionine:uroporphyrinogen III methyltransferase, was sharply induced during the earliest stages of the infection (1-12 hpi) attesting to the importance of iron sequestration and metabolism.

In addition to key enzymes involved in secondary metabolite biosynthesis, transporters are also required to deploy or secrete secondary metabolites (Table 4). For example, HC-toxin is a virulence factor of Cochliobolus carbonum on maize and is synthesized by a NRPS named HTS1 [82]. It is exported from the cell by two major facilitator superfamily (MFS) transporters, TOXA and TOXB [83]. Three genes encoding transporters with 
Table 2 Description and expression of up-regulated genes involved in secondary metabolite synthesis

\begin{tabular}{|c|c|c|c|c|c|c|c|}
\hline \multirow[t]{2}{*}{ Gene ID } & \multirow[t]{2}{*}{ Description $^{a}$} & \multicolumn{6}{|c|}{ Expression level (hpi) $^{b}$} \\
\hline & & 1 & 3 & 6 & 12 & 24 & 48 \\
\hline \multicolumn{8}{|l|}{ 1. Polyketides } \\
\hline SS1G_02356 & polyketide synthase $(\mathrm{PKS} 1)^{c}$ & - & - & - & 5.1 & 7.5 & 5 \\
\hline SS1G_05681 & polyketide synthase $(\mathrm{PKS} 4)^{c}$ & - & - & 3.4 & 2.5 & - & - \\
\hline SS1G_09237 & polyketide synthase $(\mathrm{PKS} 6)^{c}$ & - & - & - & - & - & 34.3 \\
\hline SS1G_01997 & polyketide synthase $(\mathrm{PKS} 8)^{c}$ & 3.1 & 4.9 & 6.4 & 4.5 & - & - \\
\hline SS1G_11789 & polyketide synthase (PKS12) ${ }^{c}$ & - & 2.3 & - & - & - & 2.4 \\
\hline SS1G_11404 & polyketide synthase (PKS14) & 2.8 & 2.1 & 2.9 & 4.7 & 4.7 & 9.2 \\
\hline SS1G_04125 & polyketide synthase (PKS16) & 2.7 & 3 & - & - & 3.5 & - \\
\hline SS1G_05787 & polyketide synthase (PKS18) & - & - & - & - & 3.5 & 3 \\
\hline SS1G_03591 & polyketide synthase (PKS21) ${ }^{c}$ & - & - & 2.5 & - & 2.2 & - \\
\hline SS1G_04127 & polyketide synthase & 2.1 & - & - & - & 2.9 & - \\
\hline SS1G_10474 & polyketide synthase & 4 & - & - & - & 5.2 & - \\
\hline SS1G_05788 & polyketide synthase & - & - & - & 2.5 & 4.9 & 3.5 \\
\hline SS1G_11352 & DBSA oxidoreductase (FmE) & 4.4 & 2.8 & 3.3 & - & - & - \\
\hline SS1G_09239 & FAD-dependent monooxygenase (similar to BcBOA8) & - & - & - & - & 10.9 & 17.6 \\
\hline SS1G_02592 & zinc-binding oxidoreductase (ToxD) & 4 & - & - & 4.2 & 5.8 & 17.8 \\
\hline SS1G_02338 & chalcone and stilbene synthase (CHS1) & - & - & - & - & 2.1 & - \\
\hline \multicolumn{8}{|c|}{ 2. Non-ribosomal Peptides } \\
\hline SS1G_08561 & non-ribosomal peptide synthase (NRPS1) ${ }^{c}$ & - & - & - & 4.7 & 8.9 & 32.5 \\
\hline SS1G_03693 & siderophore peptide synthase (NRPS2) ${ }^{c}$ & - & 2.2 & 2.3 & - & 4 & 3.1 \\
\hline SS1G_06185 & siderophore peptide synthase (NRPS3) ${ }^{c}$ & 3.6 & 8.2 & 8.8 & 9.3 & 4.7 & - \\
\hline SS1G_10563 & non-ribosomal peptide synthase (NRPS5) ${ }^{c}$ & - & - & - & - & 2.3 & - \\
\hline SS1G_12986 & NRPS-like enzyme & - & 6.3 & 5.2 & 4.3 & 5.8 & 7 \\
\hline SS1G_09846 & NRPS-like enzyme & 2.9 & 2.2 & 2.6 & - & 2.1 & - \\
\hline SS1G_03440 & NRPS-like enzyme & - & - & - & 3.5 & 4 & - \\
\hline SS1G_00726 & NRPS-like enzyme & - & - & - & 18.8 & - & - \\
\hline SS1G_01217 & NRPS-like enzyme & - & - & - & 5.4 & 10.5 & 7.7 \\
\hline \multicolumn{8}{|l|}{ 3. Other } \\
\hline SS1G_09177 & siroheme synthase & 10.9 & 8.7 & 8.3 & 5.7 & - & - \\
\hline
\end{tabular}

${ }^{\mathrm{a} A n n o t a t i o n}$ based on the presence of conserved PFAM domains and BLAST reports

${ }^{\mathrm{b}}$ Fold change relative to $0 \mathrm{~h}$ post inoculation (hpi). (-) No significant change in expression

'Gene abbreviations used by Amselem et al., 2011 [38]

More information about the genes can be found in Additional file 3: Table S3

similarity to TOXA proteins were up-regulated in the current study. The proteins encoded by SS1G_09759, SS1G_00919 and SS1G_06662 exhibited 51\% similarity to putative HC-toxin efflux carrier TOXA from Aspergillus lentulus, $61 \%$ to the HC-toxin efflux carrier TOXA from Glarea lozoyensis and 84\% to the MFS toxin efflux pump protein of $B$. cinerea, respectively. It might therefore be inferred that these transporters are involved in the efflux of toxins into the host plant during S. sclerotiorum infection, but characterization of their precise substrate specificities requires further study.

To date, sclerin is the only selective phytotoxin reported from S. sclerotiorum. It has phytotoxic effects and causes necrotic and chlorotic tissue formation in B. napus, B. jun$c e a$, and Sinapis alba which are susceptible to sclerotinia stem rot disease, but not on a resistant species Erucastrum gallicum [77]. The genes involved in the sclerin synthesis pathway have not been reported; however, the presence of various genes encoding enzymes involved in the synthesis of known secondary metabolites in S. sclerotiorum and the comparably high levels of expression of these genes during infection suggests that, similar to B. cinerea, S. sclerotiorum has the capacity to secrete several different types of secondary metabolites. The transcriptome information reported in the current study will be useful in characterizing these secondary metabolite biosynthetic pathways. 
Table 3 Description and expression of up-regulated genes encoding cytochrome p450 enzymes

\begin{tabular}{|c|c|c|c|c|c|c|c|}
\hline \multirow[t]{2}{*}{ Gene ID } & \multirow[t]{2}{*}{ Description $^{a}$} & \multicolumn{6}{|c|}{ Expression level (hpi) ${ }^{b}$} \\
\hline & & 1 & 3 & 6 & 12 & 24 & 48 \\
\hline SS1G_02340 & cytochrome p450 (pistatin demethylase) & 3.0 & 5.4 & 6.7 & 5.1 & - & - \\
\hline SS1G_04805 & CYP51 (eburicol 14 alpha-demethylase) & 3.4 & 3.6 & 2.4 & 2.2 & - & - \\
\hline SS1G_00406 & CYP55a3 (nitric oxide reductase) & 2.1 & 2.5 & 2.1 & - & - & - \\
\hline SS1G_00623 & cytochrome p450 & 2.3 & - & - & - & - & 2.5 \\
\hline SS1G_13470 & cytochrome P450 (alkane hydroxylase) & 2.2 & - & - & - & - & - \\
\hline SS1G_05491 & cytochrome p450 & - & 86 & 271 & 495 & 1067 & 5372 \\
\hline SS1G_13909 & cytochrome P450 & - & 2.1 & 2.2 & - & - & - \\
\hline SS1G_05384 & cytochrome p450 & - & 2.6 & - & 2.4 & - & - \\
\hline SS1G_02363 & cytochrome p450 (trichodiene oxygenase) & - & - & - & 4.2 & 3.3 & 3.9 \\
\hline SS1G_04088 & cytochrome p450 (oxidoreductase) & - & - & - & 3.5 & 7.5 & 18.7 \\
\hline SS1G_11553 & cytochrome p450 & - & - & - & 2.0 & 2.3 & 2.9 \\
\hline SS1G_03436 & cytochrome p450 (benzoate 4-monooxygenase) & - & - & - & 4.0 & 2.6 & - \\
\hline SS1G_05490 & cytochrome p450 (benzoate 4-monooxygenase) & - & - & - & - & 4.6 & 8.6 \\
\hline SS1G_01843 & cytochrome p450 (benzoate 4-hydroxylase) & - & - & - & - & 5.5 & 3.0 \\
\hline SS1G_13957 & cytochrome p450 & - & - & - & - & 14.9 & 20.4 \\
\hline SS1G_02157 & cytochrome p450 & - & - & - & - & 3.6 & 9.9 \\
\hline SS1G_04780 & cytochrome p450 & - & - & - & - & 2.2 & 2.8 \\
\hline SS1G_06101 & cytochrome p450 & - & - & - & - & 4.9 & 12.7 \\
\hline SS1G_10037 & cytochrome p450 & - & - & - & - & 8.6 & 9.0 \\
\hline SS1G_11430 & cytochrome p450 (alkane hydroxylase) & - & - & - & - & 2.1 & 4.7 \\
\hline SS1G_11697 & cytochrome p450 & - & - & - & - & 2.4 & 2.5 \\
\hline SS1G_14168 & cytochrome p450 & - & - & - & - & 2.1 & 6.0 \\
\hline SS1G_11768 & cytochrome p450 & - & - & - & - & 5.3 & - \\
\hline SS1G_01006 & cytochrome p450 & - & - & - & - & - & 3.5 \\
\hline SS1G_08136 & cytochrome p450 & - & - & - & - & - & 7.0 \\
\hline SS1G_14163 & cytochrome p450 & - & - & - & - & - & 3.1 \\
\hline SS1G_08677 & cytochrome P450 (monooxygenase) & - & - & - & - & - & 2.8 \\
\hline
\end{tabular}

${ }^{a}$ Annotation based on the presence of conserved PFAM domains and BLAST reports

${ }^{\mathrm{b}}$ Fold change relative to $0 \mathrm{~h}$ post inoculation (hpi). (-) No significant change in expression

More information about the genes can be found in Additional file 3: Table S3

\section{Detoxification}

Plant pathogens must contend with various host biochemical defense mechanisms during the infection process. This can be achieved through avoidance (e.g. intracellular growth of pathogens to avoid extracellular phytoalexins), resistance (e.g. mutations that alter sensitivity to antimicrobial compounds) or detoxification (e.g. modification or degradation of host phytoalexins). The energy-dependent efflux of toxic phytochemicals by membrane-associated transporters is a general detoxification mechanism that is common in pathogens with broad host ranges [84]. A total of 33 genes encoding ATP-binding cassette (ABC) transporters and 218 major facilitator superfamily (MFS) transporters have been identified in the $S$. sclerotiorum genome [38]. As a group, the ABC and MFS transporters exhibit a wide range of specificities (polysaccharides, drugs, sugars, heavy metals, peptides, amino acids and inorganic ions); however, some have been implicated in the secretion of fungal toxins or the efflux of host phytoalexins [85].

In total, genes encoding 91 MFS transporters and 14 $\mathrm{ABC}$ transporters were up-regulated in the current study (Table 4). The contribution of $\mathrm{ABC}$ transporters to the ability of pathogens to tolerate phytoalexins is well known. In $B$. cinerea, the gene encoding the $A B C$ transporter BcAtrB was induced during the early stages of infection and in the presence of camalexin. Inactivation of the $B c A t r B$ gene lead to increased sensitivity to camalexin and reduced virulence [86]. BcAtrB has also been implicated in the transport of the phytoalexin resveratrol, certain antibiotics and fungicides, as well as the plant phenylpropanoid eugenol [87, 88]. The S. sclerotiorum orthologue of BcAtrB (SS1G_13659) was also up-regulated at 24-48 hpi 
Table 4 Description and expression of up-regulated genes encoding transporters

\begin{tabular}{|c|c|c|c|c|c|c|c|}
\hline \multirow[t]{2}{*}{ Gene ID } & \multirow[t]{2}{*}{ Description $^{a}$} & \multicolumn{6}{|c|}{ Expression level (hpi) ${ }^{b}$} \\
\hline & & 1 & 3 & 6 & 12 & 24 & 48 \\
\hline \multicolumn{8}{|c|}{$\begin{array}{l}\text { 1. ABC transporter superfamily } \\
\text { 1.1 Pleiotropic Drug Resistance }\end{array}$} \\
\hline SS1G_06715 & Pleiotropic Drug Resistance (PDR) & 2.0 & - & - & - & - & - \\
\hline SS1G_13659 & pleiotropic drug resistance-like (PDR) & - & - & - & - & 3.1 & 13.5 \\
\hline SS1G_04483 & pleiotropic drug resistance-like (PDR) & - & - & - & - & - & 4.6 \\
\hline \multicolumn{8}{|c|}{ 1.2 Multi Drug Resistance } \\
\hline SS1G_04756 & $\mathrm{ABC}$ multidrug transporter & 3.4 & 7.9 & 6.5 & 3.7 & 2.4 & - \\
\hline SS1G_07287 & $\mathrm{ABC}$ multidrug transporter & 3.5 & 3.2 & 2.6 & - & - & 2.2 \\
\hline SS1G_03324 & $\mathrm{ABC}$ multidrug transporter & 3.5 & 4.8 & 4.4 & - & - & - \\
\hline SS1G_13112 & $\mathrm{ABC}$ multidrug transporter & - & 4.1 & 6.2 & 3.9 & - & - \\
\hline SS1G_02000 & $A B C$ multidrug transporter & - & - & 3.9 & 3.2 & - & - \\
\hline SS1G_06062 & $A B C$ multidrug transporter & - & - & - & - & 2.4 & 2.8 \\
\hline \multicolumn{8}{|l|}{ 1.3 Other families } \\
\hline SS1G_04757 & mitochondrial ATP-binding cassette protein involved in iron homeostasis & - & 6.5 & 5.1 & 3.4 & - & - \\
\hline SS1G_07963 & $A B C$ transporter with iron-containing redox enzyme with death domains & - & - & - & - & 4.0 & 8.5 \\
\hline SS1G_12070 & ABC lipid transporter & - & - & - & - & - & 2.6 \\
\hline SS1G_10747 & ABC peroxysomal fatty Acyl CoA transporter & - & - & - & - & - & 3.2 \\
\hline SS1G_05904 & ABC peroxysomal fatty Acyl CoA transporter & - & - & - & - & - & 2.0 \\
\hline \multicolumn{8}{|c|}{ 2. MFS transporter superfamily } \\
\hline \multicolumn{8}{|c|}{ 2.1 Efflux pumps and drug resistance } \\
\hline SS1G_10155 & MFS drug efflux and drug resistance & 10.1 & 5.5 & 3.7 & - & - & - \\
\hline SS1G_11948 & MFS drug efflux and drug resistance & 2.8 & 6.7 & 5.5 & - & - & - \\
\hline SS1G_00919 & MFS gliotoxin efflux transporter & - & 2.7 & 3.0 & - & - & - \\
\hline SS1G_09759 & MFS aflatoxin efflux transporter & - & 8.0 & - & - & - & - \\
\hline SS1G_05556 & MFS drug efflux and drug resistance & - & - & 2.4 & - & - & - \\
\hline SS1G_06662 & MFS toxin efflux pump & - & - & 3.0 & - & - & - \\
\hline SS1G_05572 & MFS drug resistance transporter & 11.4 & 2.9 & - & - & - & - \\
\hline SS1G_02358 & MFS drug resistance transporter & - & - & - & 4.1 & 3.7 & 4.3 \\
\hline SS1G_12101 & MFS drug resistance transporter & - & - & - & - & 2.4 & - \\
\hline SS1G_02394 & MFS drug resistance transporter & - & - & - & - & - & 4.6 \\
\hline SS1G_05756 & MFS drug resistance transporter & - & - & - & - & - & 15.2 \\
\hline SS1G_10566 & MFS drug resistance transporter & - & - & - & - & - & 5.5 \\
\hline SS1G_05556 & MFS drug resistance transporter & - & - & - & - & - & 2.0 \\
\hline SS1G_05095 & MFS fungal trichothecene efflux pump (TRI12) & - & - & - & - & - & 7.1 \\
\hline SS1G_05145 & MFS fungal trichothecene efflux pump (TRI12) & - & - & - & - & 5.0 & - \\
\hline SS1G_13024 & MFS drug resistance transporter & - & - & - & - & - & 3.6 \\
\hline SS1G_11919 & MFS multidrug transporter & - & 2.7 & 2.3 & - & - & - \\
\hline SS1G_02623 & MFS multidrug transporter & - & - & - & - & - & 4.7 \\
\hline SS1G_08554 & MFS multidrug transporter & - & - & - & - & - & 2.9 \\
\hline SS1G_10279 & MFS multidrug transporter & - & - & - & - & - & 2.1 \\
\hline SS1G_09892 & MFS multidrug transporter & - & - & - & - & - & 10.2 \\
\hline SS1G_02931 & MFS multidrug transporter & - & - & - & - & - & 2.8 \\
\hline SS1G_02048 & spermidine drug resistance transporter & 2.1 & - & - & - & - & 3.2 \\
\hline
\end{tabular}


Table 4 Description and expression of up-regulated genes encoding transporters (Continued)

\begin{tabular}{|c|c|c|c|c|c|c|c|}
\hline \multicolumn{8}{|c|}{ 2.2 Sugar transporters } \\
\hline SS1G_04841 & hexose transporter & 4.1 & 2.9 & - & - & - & - \\
\hline SS1G_13734 & hexose transporter HXT13 & - & 2.1 & - & - & - & - \\
\hline SS1G_04273 & hexose transporter & - & - & - & - & 6.1 & 15.8 \\
\hline SS1G_12412 & hexose transporter & - & - & 2.1 & - & 2.5 & 2.4 \\
\hline SS1G_09710 & MFS sugar transporter & - & - & 2.7 & 2.6 & 4.8 & 6.5 \\
\hline SS1G_06023 & MFS monosaccharide transporter & 6.9 & 6.7 & 7.0 & 4.9 & - & - \\
\hline SS1G_10125 & MFS monosaccharide transporter & 2.1 & 2.4 & - & - & - & - \\
\hline SS1G_06620 & MFS monosaccharide transporter & - & - & - & - & 2.4 & - \\
\hline SS1G_07618 & MFS monosaccharide transporter & - & - & - & - & - & 2.1 \\
\hline SS1G_08794 & MFS sucrose transporter & - & - & 2.6 & - & - & - \\
\hline SS1G_04208 & MFS sugar transporter & - & - & - & 4.0 & 36.7 & 49.2 \\
\hline SS1G_10413 & MFS sugar transporter & - & - & - & 2.3 & 4.6 & 4.8 \\
\hline SS1G_06751 & MFS sugar transporter & - & - & - & - & 2.0 & - \\
\hline SS1G_08117 & MFS sugar transporter & - & - & - & - & 2.8 & - \\
\hline SS1G_11591 & MFS sugar transporter & - & - & - & - & 10.7 & 9.4 \\
\hline SS1G_01523 & MFS sugar transporter & - & - & - & - & 13.1 & 12.5 \\
\hline SS1G_00928 & MFS sugar transporter & - & - & - & - & 7.2 & 10.6 \\
\hline SS1G_08467 & MFS sugar transporter & - & - & - & - & 2.9 & 2.5 \\
\hline SS1G_07132 & MFS sugar transporter & - & - & - & - & 8.3 & 6.3 \\
\hline SS1G_01759 & MFS sugar transporter & - & - & - & - & - & 2.8 \\
\hline SS1G_14316 & MFS sugar transporter & - & - & - & - & - & 6.1 \\
\hline SS1G_06402 & MFS sugar transporter & - & - & - & - & - & 16.2 \\
\hline SS1G_01302 & MFS sugar transporter & - & - & - & - & - & 47.6 \\
\hline SS1G_03579 & MFS sugar transporter & - & - & - & - & - & 4.1 \\
\hline SS1G_05006 & MFS sugar transporter & - & - & - & - & - & 11.8 \\
\hline SS1G_08982 & MFS quinate transporter & - & - & - & - & 18.9 & 26.1 \\
\hline SS1G_09293 & MFS quinate transporter & - & - & - & - & 3.5 & 2.9 \\
\hline SS1G_08981 & MFS quinate transporter & - & - & - & - & 49.3 & 38.4 \\
\hline SS1G_00139 & MFS carbohydrate transporter & - & 3.1 & 2.2 & 2.0 & - & - \\
\hline SS1G_04149 & MFS carbohydrate transporter & - & 3.2 & 2.5 & - & - & 8.2 \\
\hline SS1G_07210 & MFS carbohydrate transporter & - & - & - & - & 2.5 & 2.3 \\
\hline SS1G_08328 & MFS carbohydrate transporter & - & - & - & - & 5.8 & 4.1 \\
\hline SS1G_13671 & MFS carbohydrate transporter & - & - & - & - & 2.4 & - \\
\hline SS1G_14028 & MFS carbohydrate transporter & - & - & - & - & 2.1 & 2.0 \\
\hline SS1G_13819 & MFS carbohydrate transporter & - & - & - & - & - & 8.1 \\
\hline SS1G_11142 & MFS monocarboxylate transporter & - & - & - & - & 3.6 & - \\
\hline SS1G_01656 & MFS monocarboxylate transporter & - & - & - & - & - & 2.2 \\
\hline SS1G_07861 & MFS galactonate transporter & 2.8 & - & - & - & - & - \\
\hline SS1G_09368 & MFS galactonate transporter & - & - & 3.6 & 5.8 & 8.3 & 9.0 \\
\hline SS1G_09803 & MFS galactonate transporter & - & - & - & 4.2 & 14.9 & 13.1 \\
\hline SS1G_01055 & MFS galactonate transporter & - & - & - & - & 3.8 & - \\
\hline SS1G_10065 & MFS galactonate transporter & - & - & - & - & 73.2 & 487 \\
\hline SS1G_11224 & MFS galactonate transporter & - & - & - & - & 3.3 & 2.2 \\
\hline SS1G_11609 & MFS galactonate transporter & - & - & - & - & 3.0 & 13.0 \\
\hline
\end{tabular}


Table 4 Description and expression of up-regulated genes encoding transporters (Continued)

\begin{tabular}{|c|c|c|c|c|c|c|c|}
\hline SS1G_12649 & MFS galactonate transporter & - & - & - & - & 6.9 & 2.9 \\
\hline SS1G_12698 & MFS galactonate transporter & - & - & - & - & 4.0 & 5.7 \\
\hline SS1G_05090 & MFS galactonate transporter & - & - & - & - & 7.2 & 5.7 \\
\hline SS1G_10896 & MFS alpha glucoside:h + symporter & - & - & - & - & 4.4 & 2.3 \\
\hline SS1G_13094 & MFS alpha glucoside:h + symporter & - & - & - & - & 5.0 & 18.6 \\
\hline SS1G_03673 & 4-hydroxyphenylacetate permease and sugar transporter & - & - & - & - & - & 5.3 \\
\hline SS1G_04849 & MFS-fucose permease & - & - & - & - & - & 3.1 \\
\hline SS1G_02117 & lactose permease & - & - & - & 2.8 & 23 & 31.3 \\
\hline SS1G_08398 & maltose permease & - & 4.2 & - & - & - & 3.8 \\
\hline SS1G_07922 & maltose permease & - & - & - & - & 2.2 & - \\
\hline SS1G_12954 & maltose permease & - & - & - & - & - & 8.2 \\
\hline \multicolumn{8}{|l|}{2.3 Oligopeptides } \\
\hline SS1G_02676 & oligopeptide transporter & 2.0 & - & - & - & 4.0 & 5.2 \\
\hline SS1G_09296 & oligopeptide transporter & - & - & - & - & 3.3 & 3.1 \\
\hline SS1G_09297 & oligopeptide transporter & - & - & - & - & - & 2.8 \\
\hline SS1G_10121 & MFS peptide transporter & 5.9 & 7.4 & 5.7 & 3.0 & - & - \\
\hline SS1G_07522 & MFS peptide transporter & - & - & 2.1 & - & - & 4.1 \\
\hline \multicolumn{8}{|l|}{2.4 others } \\
\hline SS1G_08628 & MFS transporter & 5.9 & 6.6 & 5.6 & 3.0 & 2.4 & - \\
\hline SS1G_04769 & MFS transporter & - & - & - & - & - & 7.3 \\
\hline SS1G_13941 & MFS general substrate transporter & - & 3.0 & - & - & - & - \\
\hline SS1G_13194 & MFS general substrate transporter & - & - & - & - & 2.2 & - \\
\hline SS1G_07517 & MFS vacuole effluxer Atg22 transporter & - & 3.5 & 3.5 & - & 2.7 & 2.4 \\
\hline SS1G_04978 & MFS sulfate transporter & - & - & - & - & 2.2 & - \\
\hline SS1G_12690 & MFS nitrite transporter & - & 7.0 & 12.2 & 10.7 & - & - \\
\hline SS1G_02906 & MFS nicotinic acid transporter & - & - & - & - & 3.8 & 2.1 \\
\hline \multicolumn{8}{|c|}{ 3. Amino acid transporters } \\
\hline SS1G_08387 & amino acid transporter & - & - & - & 3.0 & 10.4 & 9.5 \\
\hline SS1G_11233 & amino acid transporter & - & - & - & - & 2.7 & - \\
\hline SS1G_01802 & amino acid transporter & - & - & - & - & - & 3.9 \\
\hline SS1G_09839 & amino acid transporter & - & - & - & - & - & 13.3 \\
\hline SS1G_14102 & choline, amino acid permease transport & - & - & - & 2.3 & - & - \\
\hline SS1G_05293 & choline, amino acid permease transport & - & - & - & - & - & 5.0 \\
\hline SS1G_06535 & choline, amino acid permease transport & - & - & - & - & - & 5.1 \\
\hline SS1G_06536 & choline, amino acid permease transport & - & - & - & - & - & 3.1 \\
\hline SS1G_04884 & ammonium transporter & - & - & - & - & 6.8 & - \\
\hline SS1G_11781 & arginine permease & 2.2 & - & - & - & - & - \\
\hline SS1G_11563 & amino acid permease & 3.2 & 4.7 & 4.9 & 5.5 & - & - \\
\hline SS1G_02549 & amino acid permease & 3.1 & 4.2 & 3.9 & 2.2 & - & - \\
\hline SS1G_14381 & amino acid permease & 2.6 & 4.6 & 3.8 & 3.3 & - & - \\
\hline SS1G_13916 & amino acid permease & 9.1 & 11.5 & 8.6 & 6.9 & 2.3 & - \\
\hline SS1G_10633 & amino acid permease & - & 2.1 & 2.7 & 2.1 & 2.4 & 4.9 \\
\hline SS1G_03403 & amino acid permease & - & - & - & - & 3 & - \\
\hline SS1G_11780 & amino acid permease & - & - & - & - & - & 2.9 \\
\hline SS1G_06841 & amino acid permease & - & - & - & - & - & 2.7 \\
\hline
\end{tabular}


Table 4 Description and expression of up-regulated genes encoding transporters (Continued)

\begin{tabular}{|c|c|c|c|c|c|c|c|}
\hline \multicolumn{8}{|l|}{ Others } \\
\hline SS1G_03654 & formate nitrate transporter & 3.0 & 3.2 & 3.1 & 3.4 & 4.0 & 3.9 \\
\hline SS1G_09621 & mitochondrial phosphate carrier protein & 3.8 & 5.0 & 4.4 & 3.3 & - & 3.0 \\
\hline SS1G_01720 & C4-dicarboxylate transporter & 20.2 & 19.0 & 18.8 & 8.5 & - & - \\
\hline SS1G_02619 & C4-dicarboxylate transporter & 2.2 & 2.7 & 2.6 & - & - & - \\
\hline SS1G_12337 & calcium proton exchanger & 2.5 & 3 & 2.2 & - & - & 2.6 \\
\hline SS1G_02802 & putative $\mathrm{Mg} 2+$ transporter & 2.6 & 2.8 & 2.4 & - & - & 3.8 \\
\hline SS1G_02548 & UDP-N-acetylglucosamine transporter & 2.4 & 2.8 & 2.8 & - & - & - \\
\hline SS1G_11564 & lysosomal cystine transporter & 2.1 & 3.0 & 2.5 & - & - & - \\
\hline SS1G_06298 & cation efflux family transporter & 3.1 & 4.0 & 3.5 & - & - & - \\
\hline SS1G_09822 & monocarboxylate permease-like protein & 2.1 & 2.5 & - & - & - & 2.7 \\
\hline SS1G_06910 & vacuolar iron transporter & 2.7 & - & 2.3 & - & 3.1 & 4.0 \\
\hline SS1G_03019 & carnitine mitochondrial carrier protein & 2.4 & - & 2.6 & - & - & 3.4 \\
\hline SS1G_08146 & AGZA family xanthine/uracil permease & 3 & - & - & - & - & - \\
\hline SS1G_10280 & polyamine transport protein & - & 2.7 & 2.1 & - & - & - \\
\hline SS1G_04686 & phosphate/sulfate permease & - & 4.2 & - & - & 3.3 & - \\
\hline SS1G_12991 & cation, potassium transporter & - & - & 2.4 & - & - & - \\
\hline SS1G_08795 & succinate fumarate, mitochondrial transporter & - & - & 5.4 & - & - & 8.0 \\
\hline SS1G_06006 & purine-cytosine permease & - & - & - & 6.4 & 3.7 & - \\
\hline SS1G_03305 & solute carrier protein & - & - & - & 3.0 & - & - \\
\hline SS1G_11712 & cation, magnesium transporter & - & - & - & - & 2.4 & - \\
\hline SS1G_07645 & cation, potassium transporter & - & - & - & - & 2.2 & 2.5 \\
\hline SS1G_02434 & mitochondrial 2-oxoglutarate malate carrier protein & - & - & - & - & 45.3 & 44.7 \\
\hline SS1G_06806 & 3-oxoacyl-[acyl-carrier protein] reductase & - & - & - & - & 3.7 & 5.3 \\
\hline SS1G_10890 & acetyl-CoA acetyltransferases & - & - & - & - & 3.0 & 3.4 \\
\hline SS1G_04606 & arsenite efflux transporter & - & - & - & - & - & 2.8 \\
\hline SS1G_01231 & sulfate permease & - & - & - & - & - & 5.7 \\
\hline SS1G_01111 & cytosine permease & - & - & - & - & - & 3.6 \\
\hline SS1G_10204 & mitochondrial carrier protein & - & - & - & - & - & 2.2 \\
\hline SS1G_06998 & peroxisomal, mitochondrial carrier protein & - & - & - & - & - & 2.0 \\
\hline SS1G_04019 & mitochondrial carrier protein & - & - & - & - & - & 2.5 \\
\hline SS1G_08663 & solute carrier protein & - & - & - & - & - & 2.5 \\
\hline SS1G_05281 & fatty acid transporter involves in acyl-CoA synthetase) & - & - & - & - & - & 4.3 \\
\hline SS1G_06664 & nucleoside transporter & 3.7 & 5.7 & 5.7 & 4.3 & 2.8 & - \\
\hline SS1G_06212 & nucleoside transporter & 3.2 & - & - & - & - & - \\
\hline SS1G_04537 & nucleoside transporter & - & - & - & - & 2.6 & - \\
\hline SS1G_09667 & nucleoside transporter & - & - & - & - & - & 2.3 \\
\hline
\end{tabular}

${ }^{a}$ Annotation based on the presence of conserved PFAM domains and BLAST reports

${ }^{b}$ Fold change relative to $0 \mathrm{~h}$ post inoculation (hpi). (-) No significant change in expression

More information about the genes can be found in Additional file 3: Table S3

in the current study and is likely to play a similar role in phytoalexin avoidance. While BcAtrA was not associated with $B$. cinerea virulence on bean, it is believed to be a multidrug transporter based on its ability to reduce the sensitivity of yeast to cycloheximide and catechol [89]. The S. sclerotiorum orthologue of BcAtrA (SS1G_06715) was slightly induced at $1 \mathrm{hpi}$. The ABC transporter AtrD has been implicated in resistance to demethylation inhibitor fungicides in B. cinerea [90] and S. homeocarpa [91], but the S. sclerotiorum orthologue (SS1G_02407) was not upregulated during $B$. napus infection in the current study. The S. sclerotiorum orthologue of BMR1 (SS1G_04483), 
which is involved in resistance to polyoxin and iprobenfos toxicants in B. cinerea [92], was up-regulated at $48 \mathrm{hpi}$ in the current study.

BcMFS1 encodes a MFS transporter in B. cinerea which is involved in detoxification of natural toxic compounds, such as camptothecin and cercosporin, and provides resistance to some fungicides, such as demethylation inhibitors (DMI) [93]. SS1G_12842 is orthologous to BcMFS1 and was expressed, but not up-regulated, in the current study. Some of the MFS transporters up-regulated in the current study may be involved in detoxification, but this needs further investigation.

While transport of phytoalexins from the pathogen provides a mechanism to avoid the effects of host phytoalexins, enzymes may also be employed to permanently inactivate these compounds or transform them to a less toxic state. The gene encoding brassinin glucosyltransferase 1 (SsBGT1) (SS1G_09997) was up-regulated during infection of B. napus cultivar Surpass 400 leaves by S. sclerotiorum isolate UQ1280 [94]. SsBGT1 was induced by plant phytoalexins, such as brassinin, and involved in detoxification of plant defense compounds via glucosylation [94]. This gene was also up-regulated at 24 and 48 hpi with expression levels 28 and 223-fold greater than the inoculum, respectively, in S. sclerotiorum 1980 in the current study.

The plant pathogen Nectria haematococca demethylates and detoxifies the pea phytoalexin, pisatin, by means of pisatin demethylase (PDA), a cytochrome P450 enzyme [95]. As such, PDA contributes to virulence of this fungus on pea [96]. SS1G_02340 encodes a cytochrome P450 enzyme (Table 3) with similarity to PDA from other plant pathogens, such as Penicillium chrysogenum and Verticillium dahliae, and was up-regulated at 1 to $12 \mathrm{hpi}$ in the current study. It may be involved in the detoxification of structurally similar phytoalexins from B. napus.

Plant pathogens degrade aromatic compounds produced by plant defense systems, such as benzoic acid derivatives arising from the $\beta$-ketoadipate pathway [97]. The CYP53 family of cytochrome P450 enzymes play an essential role in this pathway through the hydroxylation of benzoic acid to 4-hydroxybenzoate. The first report of CYP53A1 enzyme function was from Aspergillus niger [98]. The enzyme encoded by the Cochliobolus lunatus orthologue of this gene, CYP53A15, was capable of para hydroxylation of benzoate [99]. The SS1G_01843 gene encodes a benzoate 4-hydroxylase and was up-regulated at 24 and $48 \mathrm{hpi}$ in the current study and it may have a role in detoxification.

Propiconazole is a type of sterol DMI fungicide which inhibits the biosynthesis of ergosterol by targeting CYP51 (eburicol 14 alpha-demethylase) [100]. CYP51 is involved in the biosynthesis of fungal sterols which are required for membrane stability [101]. It has been speculated that overexpression of CYP51 or its paralogues in S. homoeocarpa and Monilinia fructicola is one of the mechanisms that decrease sensitivity to DMI [91, 102]. SS1G_04805 is the paralogue of $S$. homoeocarpa CYP51 and was up-regulated at 1 to $12 \mathrm{hpi}$ in the current study. It may play a similar role in detoxifying phytoalexins.

Glucosinolates, found mainly in the Brassicaceae, and the more ubiquitous hydroxynitrile glycosides, are important plant defense compounds. These compounds undergo enzymatic transformation to release a wide variety of toxic metabolites upon tissue damage, including hydrogen cyanide, a potent inhibitor of cell respiration [103]. Cyanide hydratases were reported to have a role in detoxifying hydrogen cyanide in B. cinerea [104] and Leptosphaeria maculans [105]. In the current study, four genes encoded cyanide hydratases or cyanate hydrolases, SS1G_13754, SS1G_10174, SS1G_01652 and SS1G_11485 (Table 5), were significantly up-regulated during different infection stages from 1 to $48 \mathrm{hpi}$. These may be important for the detoxification of metabolites generated from glucosinolates during infection of B. napus.

Glutathione S-transferases (GST) are best known for their ability to conjoin the reduced form of glutathione to xenobiotic chemicals leading to their detoxification. Several genes encoding GSTs were significantly induced during different infection from 1 to 48 hpi with the majority being induced at 24 hpi (Table 5). SS1G_01918 was highly induced from 1 to 48 hpi with a peak expression level 189-fold greater than the inoculum. GSTs were first reported in plants because of their ability to detoxify herbicides [106]. Xenobiotic detoxification by this group of enzymes has also been reported in other fungi, such as Phanerochaete chrysosporium [107]. Bcgst1 from $B$. cinerea was the first GST reported in filamentous fungi; however, disruption of the Bcgst1 gene indicated that the enzyme did not play a role in virulence [108]. Bcgst1 has been suggested to be a potential virulence factor as it is involved in tolerance against plant defense compounds, but the exact mechanism remains to be investigated. The orthologue of Bcgst1 in S. sclerotiorum (SS1G_07195) was up-regulated at 24 and $48 \mathrm{hpi}$ in the current study.

2-Nitropropane dioxygenase is an enzyme that catalyzes the oxidation of nitroalkanes, such as 2-nitropropane, into carbonyl compounds and nitrite. Nitroalkanes are toxic compounds [109] and 2-nitropropane dioxygenase is involved in detoxification of nitroalkanes in the Trichoderma harzianum-tomato interaction [110]. 2-Nitropropane dioxygenase is also involved in xenobiotic degradation by Pseudomonas jeseenii [111]. In the current study, four genes encoded 2-nitropropane dioxygenases, SS1G_10881, SS1G_14466, SS1G_00355 and SS1G_11235 (Table 5), were significantly up-regulated at different infection times from 1 to $48 \mathrm{hpi}$. These may be important for the detoxification of toxic nitroalkanes during infection of $B$. napus. 
Table 5 Description and expression of up-regulated genes involved in detoxification

\begin{tabular}{|c|c|c|c|c|c|c|c|}
\hline \multirow[t]{2}{*}{ Gene ID } & \multirow[t]{2}{*}{ Description $^{a}$} & \multicolumn{6}{|c|}{ Expression level (hpi) ${ }^{b}$} \\
\hline & & 1 & 3 & 6 & 12 & 24 & 48 \\
\hline SS1G_01918 & glutathione S-transferase & 19.1 & 24.8 & 34.6 & 45.0 & 189.0 & 19.1 \\
\hline SS1G_10108 & glutathione S-transferase & - & - & - & 3.5 & 4.1 & 2.3 \\
\hline SS1G_08210 & glutathione S-transferase & - & - & - & - & 2.6 & - \\
\hline SS1G_04914 & glutathione S-transferase & - & - & - & - & 2.1 & - \\
\hline SS1G_07195 & glutathione S-transferase & - & - & - & - & 2.7 & 2.8 \\
\hline SS1G_14440 & glutathione S-transferase & - & - & - & - & 4.5 & 3.7 \\
\hline SS1G_09479 & glutathione S-transferase & - & - & - & - & - & 40.1 \\
\hline SS1G_08258 & glutathione S-transferase with glutathionyl-hydroquinone reductase, ECM4 domain & - & - & - & - & 3.1 & 2.4 \\
\hline SS1G_09997 & UDP-glucuronosyl and udp-glucosyltransferase (brassinin) & - & - & - & - & 28.0 & 223.2 \\
\hline SS1G_03517 & UDP-glucoronosyl and udp-glucosyltransferase family protein & 4.5 & 3.7 & - & 5.0 & 8.2 & 10.8 \\
\hline SS1G_13524 & glucosyltransferase family & 6.3 & 4.9 & 4.2 & 2.5 & - & 2.9 \\
\hline SS1G_13754 & nitrilase-cyanide hydratase & 3.2 & 3.5 & 3.1 & 2.2 & 4.6 & 4.4 \\
\hline SS1G_10174 & nitrilase-cyanide hydratase & - & 11.6 & 16.3 & 19.7 & 29.8 & 58.6 \\
\hline SS1G_01652 & nitrilase-cyanide hydratase & - & - & - & - & 3.4 & 2.3 \\
\hline SS1G_11485 & cyanate lyase, cyanase superfamily & - & - & - & - & 2.4 & - \\
\hline SS1G_10881 & 2-nitropropane dioxygenase & 2.8 & - & - & - & - & - \\
\hline SS1G_14466 & 2-nitropropane dioxygenase & 2.8 & 4.0 & 2.5 & - & - & 3.0 \\
\hline SS1G_00355 & 2-nitropropane dioxygenase & - & - & - & - & 3.4 & 5.4 \\
\hline SS1G_11235 & 2-nitropropane dioxygenase & - & - & - & - & 2.7 & 3.5 \\
\hline
\end{tabular}

${ }^{a}$ Annotation based on the presence of conserved PFAM domains and BLAST reports

${ }^{b}$ Fold change relative to $0 \mathrm{~h}$ post inoculation (hpi). (-) No significant change in expression

More information about the genes can be found in Additional file 3: Table S3

\section{Oxalic acid production}

Oxalic acid (OA) is crucial for $S$. sclerotiorum infection and is required for suppression of host defenses [15], regulation of hydrolytic enzyme synthesis and activity [112], and the induction of processes leading to host colonization [113] and tissue necrosis [16, 27]. OA can be synthesized from a variety of precursors, but in fungi the most common mechanism is through the hydrolysis of oxaloacetate to form OA and carbon dioxide. An oxaloacetate acetyl hydrolase $(\mathrm{OAH})$ has been characterized in S. sclerotiorum [114] and disruption of the $O A H$ gene in Aspergillus niger, B. cinerea [115] and S. sclerotiorum leads to loss of oxalic acid production and reduced virulence [116]. In the current study, the S. sclerotiorum $O A H$ gene (SS1G_08218) was expressed from $1 \mathrm{hpi}$, but only up-regulated at $48 \mathrm{hpi}$ with 5 - fold greater levels than the inoculum. $O A H$ expression was also detected in the S. sclerotiorum- P. vulgaris system, but at 6 and 72 hpi, with relatively higher expression levels at the later time [40]. Interestingly, the gene encoding oxalate decarboxylase (SS1G_10796), an enzyme that degrades OA [117], was also induced at 24 and $48 \mathrm{hpi}$ at 10 and 7 -fold greater than the inoculum, respectively. Simultaneous expression of these genes with contrasting roles fits well with the biological functions of $\mathrm{OA}$ which are dependent on a balance between the biosynthesis and decomposition of OA to tightly regulate $\mathrm{OA}$ levels through the course of infection [27]. The results of the current study showing concurrent expression of $O A H$ and the gene encoding oxalate decarboxylase are in agreement with Amselem et al. [38]. The acidic environment produced by OA has a significant role in virulence/ pathogenesis of S. sclerotiorum. Release of OA causes a reduction in ambient $\mathrm{pH}$, which stimulates hydrolytic enzyme production throughout the course of the infection $[112,118]$, as well as sclerotogenesis during its final stages $[7,119]$, though OA may not be the sole determinant affecting tissue acidification [120]. Interestingly, finetuning of OA levels through the activity of OA biogenic [121] and degradative [116] enzymes appears to be critical for early host-pathogen interactions as well, including compound appressorium formation and lesion expansion.

\section{Generation of reactive oxygen species}

Reactive oxygen species (ROS), including superoxide, hydrogen peroxide $\left(\mathrm{H}_{2} \mathrm{O}_{2}\right)$ and hydroxyl radicals, are produced by all aerobic organisms [122]. Plants generate ROS as part of the defense response against pathogen attack [123], but they may also be involved in cell proliferation and differentiation, signal transduction and ion 
transport [124]. Nicotinamide adenine dinucleotide phosphate (NADPH) oxidases (NOX) are key enzymes in oxidative burst activation resulting in ROS production $[8,125]$. NOXs produce superoxide, an important precursor of several ROS, which is then converted to $\mathrm{H}_{2} \mathrm{O}_{2}$ by superoxide dismutase [125].

In filamentous fungi, NOX enzymes are involved in various aspects of differentiation, such as sexual reproduction and the formation of penetration structures [126]. In B. cinerea both BcNOXA and BcNOXB enzymes are involved in the formation of sclerotia and pathogenicity, while BcNOXB has been specifically implicated in events leading to penetration and BcNOXA in the spreading of lesions [125]. Importantly, NOX enzymes in $B$. cinerea do not play a role in ROS production [125]. Two genes encoding S. sclerotiorum NADPH oxidases, SsNOX1 (SS1G_05661) and SsNOX2 (SS1G_11172), have been reported [8]. SsNOX1 is important for both virulence and fungal development and is also connected to oxalate production [8]. SsNOX1 and SsNOX2 were expressed, but not up-regulated in the current study. Interestingly, Kim et al. [8] proposed that the bicupin domain enzyme encoded by SS1G_10796 may be an oxalate oxidase. Oxalate oxidase catalyzes the generation of hydrogen peroxide $\left(\mathrm{H}_{2} \mathrm{O}_{2}\right)$ from oxalate [8] suggesting that ROS generated by $S$. sclerotiorum during pathogenesis may be oxalate-mediated. SS1G_10796 was up-regulated at 24 and $48 \mathrm{hpi}$ in the current study which coincided with the beginning of necrotic stage. This is in agreement with the previous study that oxalate-induced $\mathrm{H}_{2} \mathrm{O}_{2}$ in the host has a role in programmed cell death [16].

\section{Signaling}

6.1 Transcription factors Transcription factors (TFs) are essential players in the regulatory networks that govern developmental processes and the deployment of pathogenicity factors during infection. In the current study, many genes encoding diverse putative TFs were up-regulated at different stages of the infection from 1 to 48 hpi (Table 6). Several of these encoded zinc-binding TFs from the major families prevalent in fungi, namely, Cys2 His2, Cys4, and Zn2 Cys6 families [127].

The gene encoding the Pac1 zinc finger domain transcription factor (SS1G_07355) was up-regulated 8-fold at $48 \mathrm{hpi}$ in the current study. Pac1 is an orthologue of Aspergillus nidulans PacC and controls $\mathrm{pH}$-sensitive gene expression. Its activity was required for the control of a variety of physiological and pathogenesis-related processes in S. sclerotiorum [7]. In response to increasing ambient $\mathrm{pH}, \mathrm{Pac} 1$ triggers $\mathrm{OA}$ biosynthesis leading a reduction in $\mathrm{pH}$; this in turn causes an increase in SsPG1 expression and promotes sclerotial development [119]. By affecting ambient $\mathrm{pH}, \mathrm{Pac1}$ is thought to play a role in $\mathrm{OA}$ and PG accumulation and therefore its activity is critical for $S$. sclerotiorum pathogenesis.

Biosynthesis of the $B$. cinerea phytotoxin, botrydial, is regulated by the Cys2 His2 zinc finger TF $B c C R Z 1$ [128], as well as upstream signaling components phospholipase $C$ (BCPLC1) and calcineurin [129]. Four genes encoding phospholipase C-like enzymes (SS1G_03548, SS1G_05073, SS1G_08747 and SS1G_13589) were up-regulated at $48 \mathrm{hpi}$ in the current study, suggesting they might be involved in secondary metabolite biosynthesis signaling pathways or events related to the later stages of the infection.

The Penicillium roqueforti Pcz1 gene encodes $\mathrm{Zn} 2$ Cys6 TF which contributes to the regulation of growth, conidiation, and conidial germination [130]. BcYOH1 from $B$. cinerea encodes a Cys2 His2 TF which is involved in the regulation of secondary metabolite synthesis [131]. A number of genes encoding zinc cluster TFs similar to $B c Y O H 1$ and $P c z 1$ were differentially expressed at various stages of infection in the current study, including SS1G_10532, SS1G_01109 and SS1G_02054 (Table 6). Several genes encoding other types of TFs involved in fungal development were also up-regulated and are discussed below.

6.2 Phosphorylation-dependent signaling The S. sclerotiorum genome contains many different types of kinases which are involved in signaling pathways, including the G protein-coupled receptor, MAP kinase, heterotrimeric $\mathrm{G}$ protein, cAMP, and $\mathrm{Ca}^{2+}$-related signaling pathways [38]. A number of genes associated with these pathways were up-regulated during infection in the current study (Table 7) and are discussed below. While the induction of genes encoding signaling pathway components is not always necessary for activation of their associated pathways, it does imply that they may be involved in more critical aspects or regulatory checkpoints during the infection process.

Two-component histidine kinases are known to be involved in regulating responses to environmental stimuli in fungi and bacteria [132]. The gene encoding the two-component sensor histidine protein kinase Shk1 (SS1G_10091) was up-regulated only at the beginning of the infection (1 hpi) in the current study. Shk1 was previously shown to have a role in hyphal growth and sclerotial formation in S. sclerotiorum, but was not required for pathogenicity on plant leaves [10].

The suite of genes encoding eukaryotic protein kinases in S. sclerotiorum has been catalogued [11]. Genes encoding two MAPKKK genes (SS1G_00606 and SS1G_10983) were up-regulated at 48 and 1-6 hpi, respectively. SS1G_00606 is an orthologue of STE11 in S. cerevisiae and belongs to the S. sclerotiorum STE11-like MAPKKK family, while SS1G_10983 is an orthologue of BCK1 in the yeast cell wall integrity pathway. Mutation of the STE7 and 
Table 6 Description and expression of up-regulated genes encoding transcription factors

\begin{tabular}{|c|c|c|c|c|c|c|c|}
\hline \multirow[t]{2}{*}{ Gene ID } & \multirow[t]{2}{*}{ Description $^{a}$} & \multicolumn{6}{|c|}{${\text { Expression level }(\mathrm{hpi})^{\mathrm{b}}}$} \\
\hline & & 1 & 3 & 6 & 12 & 24 & 48 \\
\hline \multicolumn{8}{|l|}{ 1. Zn2Cys6 (C6) } \\
\hline SS1G_06255 & Zn2 Cys6 transcription factor & 7.0 & 4.6 & 3.9 & 2.4 & - & - \\
\hline SS1G_14383 & Zn2 Cys6 transcription factor & 3.5 & 4.4 & 3.5 & 2.1 & - & - \\
\hline SS1G_08819 & Zn2 Cys6 transcription factor & 3.6 & 2.5 & 2.1 & 3.0 & - & - \\
\hline SS1G_06907 & Zn2 Cys6 transcription factor & 4.1 & 4.1 & 3.3 & - & - & - \\
\hline SS1G_02793 & Zn2 Cys6 transcription factor & 6.0 & 3.0 & - & - & - & - \\
\hline SS1G_10324 & Zn2 Cys6 transcription factor & 2.0 & - & - & - & 2.0 & - \\
\hline SS1G_02851 & Zn2 Cys6 transcription factor & 2.2 & - & - & - & - & - \\
\hline SS1G_12532 & Zn2 Cys6 transcription factor & 2.2 & - & - & - & - & - \\
\hline SS1G_12799 & Zn2 Cys6 transcription factor & - & 2.5 & 2.5 & - & - & - \\
\hline SS1G_11949 & Zn2 Cys6 transcription factor & - & 2.7 & 2.5 & - & - & - \\
\hline SS1G_00732 & Zn2 Cys6 transcription factor & - & 2.4 & - & 5.0 & - & - \\
\hline SS1G_00398 & Zn2 Cys6 transcription factor & - & 4.1 & - & - & - & 4.8 \\
\hline SS1G_01905 & Zn2 Cys6 transcription factor & - & - & - & - & 2.7 & - \\
\hline SS1G_06876 & Zn2 Cys6 transcription factor & - & - & - & - & 2.3 & - \\
\hline SS1G_00170 & Zn2 Cys6 transcription factor & - & - & - & - & 2.3 & - \\
\hline SS1G_02339 & Zn2 Cys6 transcription factor & - & - & - & - & 2.0 & - \\
\hline SS1G_07143 & Zn2 Cys6 transcription factor & - & - & - & - & 2.7 & - \\
\hline SS1G_02791 & Zn2 Cys6 transcription factor & - & - & - & - & 2.9 & 3.0 \\
\hline SS1G_00392 & Zn2 Cys6 transcription factor & - & - & - & - & 2.2 & 2.2 \\
\hline SS1G_13144 & Zn2 Cys6 transcription factor & - & - & - & - & 2.1 & 3.0 \\
\hline SS1G_02054 & Zn2 Cys6 transcription factor & - & - & - & - & 6.6 & 36.9 \\
\hline SS1G_05109 & Zn2 Cys6 transcription factor & - & - & - & - & 6.7 & 7.2 \\
\hline SS1G_05755 & Zn2 Cys6 transcription factor & - & - & - & - & 2.6 & 3.8 \\
\hline SS1G_07003 & Zn2 Cys6 transcription factor & - & - & - & - & - & 4.6 \\
\hline SS1G_10447 & Zn2 Cys6 transcription factor & - & - & - & - & - & 2.5 \\
\hline SS1G_01353 & Zn2 Cys6 transcription factor & - & - & - & - & - & 4.1 \\
\hline SS1G_11367 & Zn2 Cys6 transcription factor & - & - & - & - & - & 3.3 \\
\hline SS1G_08351 & Zn2 Cys6 transcription factor & - & - & - & - & - & 2.1 \\
\hline SS1G_03775 & Zn2 Cys6 transcription factor & - & - & - & - & - & 6.8 \\
\hline SS1G_00787 & Zn2 Cys6 transcription factor & - & - & - & - & - & 2.8 \\
\hline SS1G_01733 & Zn2 Cys6 transcription factor & - & - & - & - & - & 2.6 \\
\hline SS1G_04056 & Zn2 Cys6 transcription factor & - & - & - & - & - & 2.7 \\
\hline SS1G_11081 & Zn2 Cys6 transcription factor & - & - & - & - & - & 2.8 \\
\hline SS1G_09741 & fungal specific transcription factor domain & 2.2 & 3.7 & 3.9 & 2.7 & - & - \\
\hline SS1G_04333 & fungal specific transcription factor domain & 2.0 & 2.3 & 2.0 & - & - & - \\
\hline SS1G_12561 & fungal specific transcription factor domain & 2.3 & 2.1 & - & - & - & - \\
\hline SS1G_09823 & fungal specific transcription factor domain & - & - & 2.1 & - & 2.2 & 2.4 \\
\hline SS1G_04846 & fungal specific transcription factor domain & - & - & - & - & 2.6 & 4.0 \\
\hline SS1G_13882 & fungal specific transcription factor domain & - & - & - & - & 2.0 & - \\
\hline SS1G_13729 & fungal specific transcription factor domain & - & - & - & - & 2.4 & - \\
\hline SS1G_02758 & fungal specific transcription factor domain & - & - & - & - & 2.5 & - \\
\hline SS1G_08406 & fungal specific transcription factor domain & - & - & - & - & 2.3 & - \\
\hline
\end{tabular}


Table 6 Description and expression of up-regulated genes encoding transcription factors (Continued)

\begin{tabular}{|c|c|c|c|c|c|c|c|}
\hline SS1G_05809 & fungal specific transcription factor domain & - & - & - & - & 3.9 & - \\
\hline SS1G_04057 & fungal specific transcription factor domain & - & - & - & - & - & 2.4 \\
\hline SS1G_11395 & fungal specific transcription factor domain & - & - & - & - & - & 2.3 \\
\hline SS1G_06361 & fungal specific transcription factor domain & - & - & - & - & - & 2.1 \\
\hline \multicolumn{8}{|l|}{ 2. Cys2His2 } \\
\hline SS1G_01109 & $\mathrm{C} 2 \mathrm{H} 2$ transcription factor & 19.8 & 26.1 & 26.6 & 15.6 & - & - \\
\hline SS1G_01684 & $\mathrm{C} 2 \mathrm{H} 2$ transcription factor & 2.6 & 3.1 & 2.7 & 2.0 & - & - \\
\hline SS1G_04676 & $\mathrm{C} 2 \mathrm{H} 2$ transcription factor & 2.0 & - & - & - & - & 7.3 \\
\hline SS1G_07355 & $\mathrm{C} 2 \mathrm{H} 2$ transcription factor ( $\mathrm{Pac} 1$ ) & - & - & - & - & - & 8.4 \\
\hline SS1G_10532 & $\mathrm{C} 2 \mathrm{H} 2$ transcription factor & - & - & - & - & - & 20.5 \\
\hline SS1G_00104 & $\mathrm{C} 2 \mathrm{H} 2$ binding site & - & - & 2.5 & 3.1 & - & 5.4 \\
\hline SS1G_09588 & $\mathrm{C} 2 \mathrm{H} 2$ binding site & - & - & - & - & 2.9 & 4.4 \\
\hline SS1G_06370 & $\mathrm{C} 2 \mathrm{H} 2$ binding site & - & - & - & - & - & 2.1 \\
\hline SS1G_09499 & $\mathrm{C} 2 \mathrm{H} 2$ binding site & - & - & - & - & - & 3.1 \\
\hline SS1G_07425 & $\mathrm{C} 2 \mathrm{H} 2$ binding site & - & - & - & - & - & 4.3 \\
\hline SS1G_06044 & $\mathrm{C} 2 \mathrm{H} 2$ binding site & - & - & - & - & - & 2.3 \\
\hline \multicolumn{8}{|c|}{ 3. basic leucine zipper (bZIP) } \\
\hline SS1G_10244 & bZIP transciption factor & 4.4 & 5.2 & - & 5.6 & - & - \\
\hline SS1G_01411 & bZIP transciption factor & - & - & - & - & 2.0 & - \\
\hline SS1G_00816 & bZIP transciption factor & - & - & - & - & - & 5.4 \\
\hline SS1G_06075 & bZIP transciption factor & - & - & - & - & - & 7.7 \\
\hline \multicolumn{8}{|l|}{ 4. Others } \\
\hline SS1G_14328 & transcription regulator BDF1 & 2.7 & 6.5 & 5.5 & 3.6 & - & - \\
\hline SS1G_11030 & CP2 transcription factor & 4.9 & 3.2 & 2.5 & - & - & - \\
\hline SS1G_04107 & MYB family transcription factor & 2.5 & 2.9 & - & - & - & - \\
\hline SS1G_06257 & pex2/pex 12 superfamily, zinc finger of C3HC4-type & 3.0 & 2.4 & - & - & - & - \\
\hline SS1G_07430 & zinc finger domain & 3.0 & - & - & - & - & 2.8 \\
\hline SS1G_03881 & $\begin{array}{l}\text { regulator of G protein signaling domain protein (RGS) } \\
\text { (GTPase activating proteins (GAPs) }\end{array}$ & 2.0 & - & - & - & - & - \\
\hline SS1G_14385 & CBF/Mak21 transcription factor & 2.4 & - & - & - & - & - \\
\hline SS1G_03280 & helix-loop-helix dna-binding protein $(H \mid H)$ & 2.4 & - & - & - & - & - \\
\hline SS1G_10206 & $\mathrm{CHY}$ and ring, zinc finger protein & - & 2.1 & - & - & - & - \\
\hline SS1G_04050 & NF-X1 zinc finger transcription factor & - & 3.0 & - & - & - & - \\
\hline SS1G_11663 & $\begin{array}{l}\text { large tegument protein UL } 36 \text { and similar to TFIIIC } \\
\text { transcription initiation factor complex subunits Tfc3 }\end{array}$ & - & - & 2.1 & 2.0 & - & - \\
\hline SS1G_03992 & transcription mediator subunit Med12 & - & - & 2.2 & - & - & - \\
\hline SS1G_13930 & Bro1-Alix- like domain and pH-response regulator protein & - & - & - & - & 2.3 & - \\
\hline SS1G_07542 & RING-H2 zinc finger protein & - & - & - & - & 2.3 & - \\
\hline SS1G_06124 & mads-box mef2 type transcription factor (SRF type) & - & - & - & - & - & 3.5 \\
\hline SS1G_03098 & homeobox transcription factor & - & - & - & - & - & 2.2 \\
\hline SS1G_03835 & homeobox $\mathrm{C} 2 \mathrm{H} 2$ transcription factor & - & - & - & - & - & 3.1 \\
\hline SS1G_06987 & yippee zinc-binding protein & - & - & - & - & - & 2.4 \\
\hline SS1G_01859 & MYB dna-binding containing domain protein & - & - & - & - & - & 6.2 \\
\hline SS1G_08831 & (vWA) transcription factor (Von Willebrand factor type A) & - & - & - & - & - & 2.3 \\
\hline SS1G_09890 & RAP transcription factor & - & - & - & - & - & 2.6 \\
\hline
\end{tabular}


Table 6 Description and expression of up-regulated genes encoding transcription factors (Continued)

\begin{tabular}{|c|c|c|c|c|c|c|c|}
\hline SS1G_02756 & transcription factor protein & - & - & - & - & - & 8.5 \\
\hline SS1G_10304 & tetratricopeptide (TPR) repeat transcriptional corepressor & - & - & - & - & - & 2.3 \\
\hline SS1G_13511 & $\begin{array}{l}\text { lipopolysaccharide (LPS)-induced transcription factor and } \\
\text { LITAF-like zinc ribbon domain }\end{array}$ & - & - & - & - & - & 2.7 \\
\hline
\end{tabular}

${ }^{a}$ Annotation based on the presence of conserved PFAM domains and BLAST reports

${ }^{b}$ Fold change relative to $0 \mathrm{~h}$ post inoculation (hpi). (-) No significant change in expression

More information about the genes can be found in Additional file 3: Table S3

STE11 orthologues in B. cinerea [133] or MST7 and MST11 in M. grisea [134] disrupted the formation of infection structures leading to loss of pathogenicity. SS1G_10021 belongs to the CAMK1 family in S. sclerotiorum and is orthologous to the S. cerevisiae CMK1/ CMK2 kinases. SS1G_10021 was up-regulated at 48 hpi in the current study. CAMKs were reported to have a role in the regulation of cell wall integrity and the response to oxidative stress [135]. A gene (SS1G_03455) orthologous to $S$. cerevisiae SAT4/HAL4 was induced at 24-48 hpi in this study. HAL family kinases play a role in the regulation of membrane permeases which are responsible for amino acid and glucose transport [136]. Genes encoding two other protein kinases, SS1G_09511 and SS1G_06542, were both significantly up-regulated at 48 hpi in the current study. The kinase encoded by SS1G_09511 has some similarity to PHO85 (SS1G_07226), a cyclin-dependent kinase involved in the regulation of cell division in response to environmental stresses [137]. SS1G_06542 is an ortholog of SHA3/SKS1, which is involved in integration of the response to glucose with hyphal development [138]. The FunK1 protein kinases are similar to eukaryotic protein kinases, but are only found in multicellular fungi [139]. The S. sclerotiorum genome contains three members of the FunK1 family (SS1G_09355, SS1G_12423 and SS1G_14212), all of which were up-regulated at the earliest stages of the infection.

SS1G_06571, SS1G_03234 and SS1G_10333 encode proteins annotated as having GTPase or GTP-binding activity and were first up-regulated at 1,1 and $48 \mathrm{hpi}$, respectively. In S. sclerotiorum, the small GTPase Rap-1 is involved in mediating the inhibitory actions of cAMP on the SMK1 MAPK signaling cascade and events leading to sclerotial development [9]. GTP-binding proteins belonging to the Ras superfamily also play a role in MAPK inhibition as effectors acting downstream of cAMP [9]. Other studies have shown that in addition to Ras, other small GTPases, such as Rap-1 and Rho/Rac/ Cdc42, also have important roles in transmitting signals via activation of MAPK cascades [140].

Protein dephosphorylation is also employed to both activate and attenuate kinase-dependent signaling pathways. Calcineurin, a Type $2 \mathrm{~B}$ serine/threonine phosphatase is required for proper sclerotial formation and hyphal cell wall formation [12]. The Type 2A serine/threonine phosphatase (PP2A) encoded by SS1G_08489 (PPH1) was shown to play a role in several aspects of $S$. sclerotiorum pathogenesis including hyphal growth, infection cushion formation, sclerotia development and synthesis of secondary metabolites such as melanin [13]. The genes encoding calcineurin and PPH1 were not upregulated in the current study; however, a possible PPH1 paralogue (SS1G_08513) which encodes a serine/threonine phosphatase with a PP2A catalytic subunit was upregulated at $48 \mathrm{hpi}$. It is possible that SS1G_08513 complements the function of PPH1in the S. sclerotiorum infection process, but characterization of its precise roles awaits further study. The SMK1 MAPK also positively regulates PPH1 activity through a nitrous oxide-dependent mechanism [13].

\section{Development}

As is the case in most multi-cellular pathogens, S. sclerotiorum undergoes dramatic morphological and biochemical changes as it passes through the various stages of the infection process. The S. sclerotiorum genome contains orthologues of $M$. oryzae genes that are involved in infection structure production and penetration [38]. Among them, SS1G_13339, SS1G_10311 and SS1G_11468 were upregulated in the current study (Table 8). SS1G_10311 and SS1G_11468 are orthologous to mas2 and mas3 in M. ory$z a e$, respectively, where they play a role in appressoria formation during the very early stages of infection [141]. However, in the current study SS1G_10311 was upregulated at 3 and $12 \mathrm{hpi}$ and SS1G_11468 was upregulated at 6-48 hpi, while SS1G_13339 was induced only at $48 \mathrm{hpi}$, suggesting that they may have alternate roles in this necrotrophic pathogen.

Morphological changes can also be triggered by environmental cues. The enzyme $\gamma$-glutamyl transpeptidase regulates glutathione levels and in turn cellular redox potential. In S. sclerotiorum, the $\gamma$-glutamyl transpeptidase encoded by SS1G_14127 (SsGgt1) plays a role in the production of compound appressoria during host penetration as well as in the development of sclerotia, but is not necessary for host colonization and symptom development [24]. In the current study, SS1G_14127 was up-regulated from 6-48 hpi, while genes encoding two other $\gamma$-glutamyl transpeptidases (SS1G_05530 and 
Table 7 Description and expression of up-regulated genes involved in signaling or gene regulation

\begin{tabular}{|c|c|c|c|c|c|c|c|}
\hline \multirow[t]{2}{*}{ Gene ID } & \multirow[t]{2}{*}{ Description $^{a}$} & \multicolumn{6}{|c|}{ 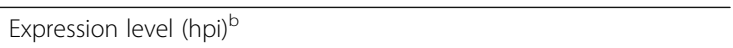 } \\
\hline & & 1 & 3 & 6 & 12 & 24 & 48 \\
\hline \multicolumn{8}{|l|}{ 1. Protein Kinases } \\
\hline SS1G_10983 & MAPKK kinase (SsBCK1) & 4.7 & 5.4 & 4.4 & 2.4 & - & - \\
\hline SS1G_11525 & protein kinase-like protein & 2.5 & 3.6 & 6.9 & 6.1 & - & - \\
\hline SS1G_00606 & MAPKK kinase (SsSTE11) & 2.1 & 2.2 & - & - & - & 2.1 \\
\hline SS1G_08085 & AGC protein kinase (SsRIM15) & - & 2.1 & 2 & - & - & - \\
\hline SS1G_03455 & HAL family protein kinase & - & - & - & - & 3.3 & 3.7 \\
\hline SS1G_06203 & CAMK protein kinase (SsRCK2) & - & - & - & - & - & 2.3 \\
\hline SS1G_10021 & CAMK protein kinase (SsCMK2) & - & - & - & - & - & 4.1 \\
\hline SS1G_06542 & ran1-like protein kinase (SsSKS1) & - & - & - & - & - & 2.3 \\
\hline SS1G_09511 & protein kinase (Ss-Other-03) & - & - & - & - & - & 5.8 \\
\hline SS1G_14212 & Funk1 serine threonine-protein kinase & 2.1 & - & - & - & - & - \\
\hline SS1G_12423 & Funk1 serine threonine-protein kinase & 2.3 & 2.4 & - & - & - & - \\
\hline SS1G_09355 & Funk1 serine threonine-protein kinase & 2.1 & 2.3 & - & - & - & - \\
\hline SS1G_10091 & two-component histidine protein kinase (SHK1) & 2.6 & - & - & - & - & - \\
\hline \multicolumn{8}{|l|}{ 2. Phosphatases } \\
\hline SS1G_11340 & tyrosine phosphatase & 2.6 & 2.1 & 2.2 & - & - & 2 \\
\hline SS1G_01711 & protein phosphatase type 1 complex subunit hex 2 reg 1 & 3.3 & 3.2 & 2.2 & - & - & - \\
\hline SS1G_04320 & tyrosine-protein phosphatase non-receptor type partial & 2.1 & 3.5 & 2.5 & - & - & - \\
\hline SS1G_06382 & protein phosphatase regulator & 2.1 & - & - & - & - & - \\
\hline SS1G_12383 & histidine acid phosphatase & - & 2.8 & - & - & - & - \\
\hline SS1G_08513 & serine threonine-protein phosphatase PP2A & - & - & - & - & - & 2.8 \\
\hline SS1G_10466 & diketo-5-methylthio-1-phosphopentane phosphatase & - & - & - & - & - & 2.5 \\
\hline \multicolumn{8}{|c|}{ 3. GTPase/GTP-binding } \\
\hline SS1G_03234 & rho GTPase activator & 3.1 & 5 & 4.6 & 4.8 & - & - \\
\hline SS1G_06571 & GTP-binding protein rho2 & 3.2 & 3 & 2.1 & - & - & - \\
\hline SS1G_01564 & nuclear GTP-binding protein NUG1 & 2.9 & - & - & - & - & - \\
\hline SS1G_04075 & ARF GTPase activator & - & - & - & - & - & 2.5 \\
\hline SS1G_10333 & GTP-binding protein & - & - & - & - & - & 2.3 \\
\hline SS1G_08371 & CLP1 GTPase & - & - & - & - & - & 2.9 \\
\hline SS1G_13589 & phosphatidyl inositol phospholipase C (PL-PLC) & - & - & - & - & - & 2.1 \\
\hline SS1G_03548 & phosphatidyl inositol phospholipase C (PL-PLC) & - & - & - & - & - & 2.1 \\
\hline SS1G_05073 & phosphatidyl inositol phospholipase C (PL-PLC) & - & - & - & - & - & 5 \\
\hline SS1G_08747 & phosphatidyl inositol phospholipase C (PL-PLC) & - & - & - & - & - & 9 \\
\hline \multicolumn{8}{|l|}{ 4. Other } \\
\hline SS1G_06667 & sir2 chromatin regulatory protein & 2.1 & 3 & 2.6 & 2.1 & - & - \\
\hline SS1G_06180 & Pal1 morphogenesis-related protein & 9.5 & 8.7 & 7.9 & 4.5 & - & - \\
\hline SS1G_04402 & Arrestin (chitin synthesis regulation) & - & - & - & - & 2.1 & - \\
\hline SS1G_03525 & ankaryin repeat protein & - & - & - & - & 2.7 & - \\
\hline SS1G_04325 & SUR7/Pal1 family (pH-response regulator) & - & - & - & - & - & 2.2 \\
\hline SS1G_10456 & SUR7/Pal1 family (pH-response regulator) & - & - & - & - & - & 12.6 \\
\hline SS1G_09665 & inositol-pentakisphosphate 2-kinase & - & - & - & - & - & 2.8 \\
\hline SS1G_00378 & SRP19 signal recognition particle protein & - & - & - & - & - & 3 \\
\hline SS1G_08048 & Sok1 cAMP-mediated signaling protein & - & - & - & - & - & 2.7 \\
\hline
\end{tabular}

${ }^{a}$ Annotation based on the presence of conserved PFAM domains and BLAST reports 
Table 8 Description and expression of up-regulated genes involved in fungal development

\begin{tabular}{|c|c|c|c|c|c|c|c|}
\hline \multirow[t]{2}{*}{ Gene ID } & \multirow[t]{2}{*}{ Description $^{a}$} & \multicolumn{6}{|c|}{ Expression level $(\mathrm{hpi})^{\mathrm{b}}$} \\
\hline & & 1 & 3 & 6 & 12 & 24 & 48 \\
\hline \multicolumn{8}{|c|}{ 1. Reserve Mobilization } \\
\hline SS1G_05192 & acid trehalase & - & - & - & - & 6.7 & 5.8 \\
\hline SS1G_01494 & 1,3-alpha-glucanase/mutanase & - & - & - & - & - & 30.7 \\
\hline SS1G_09861 & 1,3-alpha-glucanase/mutanase & - & - & - & - & - & 15 \\
\hline \multicolumn{8}{|l|}{ 2. Cell Wall } \\
\hline \multicolumn{8}{|l|}{ 2.1 Turnover } \\
\hline SS1G_05454 & chitinase & - & - & 2.4 & - & 3.5 & 2.7 \\
\hline SS1G_11700 & chitinase & - & - & - & - & 3.7 & 69.1 \\
\hline SS1G_05897 & chitinase & - & - & - & - & 3 & 2.6 \\
\hline SS1G_11304 & chitinase & - & - & - & - & - & 4.8 \\
\hline SS1G_08695 & class III chitinase & - & - & - & 5.8 & 8.2 & 11.7 \\
\hline SS1G_11212 & class III chitinase & - & - & - & - & - & 9.3 \\
\hline SS1G_12510 & class $V$ chitinase & - & - & - & - & 11.2 & 66.9 \\
\hline SS1G_09403 & alpha-N-acetylglucosaminidase & - & - & - & - & 2.9 & - \\
\hline SS1G_12837 & beta-N-acetylglucosaminidase & - & - & - & - & 2.3 & - \\
\hline SS1G_10038 & beta-N-acetylglucosaminidase & - & - & - & - & - & 3 \\
\hline SS1G_04898 & polysaccharide (chitin) deacetylase & - & - & 15.2 & - & - & 12.7 \\
\hline SS1G_01131 & polysaccharide (chitin) deacetylase & - & - & - & - & 3 & 3.6 \\
\hline SS1G_00642 & polysaccharide (chitin) deacetylase & - & - & - & - & - & 8.3 \\
\hline SS1G_12836 & N-acetylglucosamine-6-phosphate deacetylase & - & - & - & - & - & 6.7 \\
\hline SS1G_01229 & exo-beta 1,3 glucanase & - & - & - & - & 3.4 & 22.8 \\
\hline SS1G_09858 & exo-beta 1,3 glucanase & - & - & - & - & - & 2.8 \\
\hline SS1G_12930 & GPI-anchored cell wall beta-1,3-endoglucanase & 8.5 & 6.4 & 3.9 & - & - & 2.2 \\
\hline SS1G_04852 & GPI-anchored cell wall beta-endoglucanase & 2.1 & - & - & - & - & - \\
\hline \multicolumn{8}{|l|}{ 2.2 Biosynthesis } \\
\hline SS1G_04969 & glycosyl transferase (cell wall synthesis) & 2.2 & - & - & - & - & - \\
\hline SS1G_04062 & glycosyl transferase (cell wall synthesis) & - & 3.6 & 3.8 & - & - & - \\
\hline SS1G_07313 & lipopolysaccharide biosynthesis protein & - & - & - & - & - & 2.4 \\
\hline \multicolumn{8}{|l|}{ 3. Other } \\
\hline SS1G_02742 & heterokaryon incompatibility protein & 3.8 & 3.6 & 3.1 & 2.8 & - & - \\
\hline SS1G_02744 & heterokaryon incompatibility protein (SEC1) & 3.7 & 5.5 & - & 5 & 3 & 3 \\
\hline SS1G_02602 & heterokaryon incompatibility protein & 2.3 & - & - & - & - & - \\
\hline SS1G_03889 & heterokaryon incompatibility protein & - & 3.2 & - & - & - & - \\
\hline SS1G_11165 & heterokaryon incompatibility protein & - & 3.3 & 2.2 & - & - & - \\
\hline SS1G_06800 & heterokaryon incompatibility protein & - & 3.6 & - & - & - & - \\
\hline SS1G_08974 & heterokaryon incompatibility protein (WD40 repeat) & - & - & - & - & 2.7 & - \\
\hline SS1G_06855 & heterokaryon incompatibility protein & - & - & - & - & 5.2 & 2.4 \\
\hline SS1G_12973 & heterokaryon incompatibility protein & - & - & - & - & - & 2 \\
\hline SS1G_09167 & heterokaryon incompatibility protein & - & - & - & - & - & 2.5 \\
\hline SS1G_11315 & heterokaryon incompatibility protein & - & - & - & - & - & 4 \\
\hline SS1G_07526 & ferritin-like sexual development protein & 3 & 2.5 & 2.5 & - & - & 23.9 \\
\hline SS1G_04316 & acyltransferase (hard surface induced) & 2.5 & - & - & - & - & - \\
\hline SS1G_10311 & DUF cell surface protein (MAS2 orthologue) & - & 2.1 & - & 2.5 & - & - \\
\hline
\end{tabular}


Table 8 Description and expression of up-regulated genes involved in fungal development (Continued)

\begin{tabular}{|c|c|c|c|c|c|c|c|}
\hline SS1G_11468 & CAS1 appressorium specific protein (MAS3 orthologue) & - & - & 2.9 & 7.3 & 12.9 & 10.5 \\
\hline SS1G_14127 & gamma-glutamyltranspeptidase (SsGGT1) & - & - & 3.4 & 6.5 & 4.8 & 2.7 \\
\hline SS1G_05330 & gamma-glutamyltranspeptidase & - & - & - & - & 2.1 & - \\
\hline SS1G_12877 & conidiation-specific expression protein & - & - & - & - & 2.3 & 2.3 \\
\hline SS1G_12133 & predicted protein (SSP2) & - & - & - & - & 4.7 & 9.6 \\
\hline SS1G_07404 & predicted protein (Ss-Rh1) & - & - & - & - & 4.9 & 3.8 \\
\hline SS1G_01614 & G protein-coupled receptor (SOP1) & - & - & - & - & 17.7 & 17.1 \\
\hline SS1G_07626 & Velvet family & - & - & - & - & - & 5.2 \\
\hline SS1G_13339 & choline carnitine O-acyltransferase & - & - & - & - & - & 3.4 \\
\hline SS1G_02422 & UDP-galactopyranose mutase (GLF) & - & - & - & - & - & 3.9 \\
\hline SS1G_10940 & gamma-glutamyltranspeptidase & - & - & - & - & - & 4 \\
\hline SS1G_14065 & predicted protein (SSP1) & - & - & - & - & - & 47 \\
\hline
\end{tabular}

${ }^{a}$ Annotation based on the presence of conserved PFAM domains and BLAST reports

${ }^{\mathrm{b}}$ Fold change relative to $0 \mathrm{~h}$ post inoculation (hpi). (-) No significant change in expression

More information about the genes can be found in Additional file 3: Table S3

SS1G_10940) were up-regulated during the later stages at 24 and $48 \mathrm{hpi}$, respectively.

The SSP1 gene (SS1G_14065) was up-regulated 47fold at 48 hpi in the current study. The SSP2 gene (SS1G_12133), a paralogue of SSP1, was also upregulated in the later stages of the infection. SSP1 is a sclerotium-specific protein that is associated with sclerotial and apothecial development and is only detected during sclerotial formation [142]. The up-regulation of SSP1 and SSP2 at 24-48 hpi is an indication of the onset of sclerotia development at these time points in the current study. SOP1 is similar to microbial opsins, a component of the photosensory system, and is also required for sclerotial development, as well as growth and virulence in S. sclerotiorum. SOP1 was first induced at early stages of infection in A. thaliana and then more so at the sclerotial development stage (3 days post inoculation) [143]. In the current study, the gene encoding SOP1 (SS1G_01614) was highly up-regulated (17-fold) at 24 and $48 \mathrm{hpi}$ suggesting that this protein also contributes to the sclerotial development program in the S. sclerotiorum - B. napus pathosystem. Members of the velvet protein family coordinate fungal differentiation processes, including the formation of spores, sclerotia and fruiting bodies [144]. SS1G_07626 encodes a velvet protein and was induced only at $48 \mathrm{hpi}$ indicating that it may also be involved in sclerotogenesis in S. sclerotiorum. Another gene, SS1G_07404 (Ss-Rhs1, Sclerotinia sclerotiorum rearrangement hotspot repeat 1 ), was up-regulated at 24 and $48 \mathrm{hpi}$ in the current study and has been reported to be involved in sclerotial development and important for virulence in S. sclerotiorum [145].

MADS-box proteins are a conserved family of TFs and are involved in the regulation of a wide variety of functions including primary metabolism, cell cycle and cell identity
[146]. A gene encoding a MADS-box TF (SS1G_06124) was reported as being a component of the mating process in S. sclerotiorum [38] and was up-regulated 3-fold at 48 hpi in the current study. The MADS-box TF SsMADS (SS1G_05588) is required for growth and virulence [146]. Homeobox genes regulate aspects of anatomical development and in fungi are involved in hyphal growth, appressorium formation or conidia production [147]. In the current study, a gene (SS1G_03835) encoding a homeobox C2H2 TF was expressed at $48 \mathrm{hpi}$, but the precise function of this gene in S. sclerotiorum needs to be characterized. A homeobox TF (BcHOX8) that plays a role in the vegetative growth and conidiogenesis has been reported in B. cinerea [148].

In fungi, programmed cell death associated with vegetative incompatibility is determined by the interactions of proteins containing heterokaryon incompatibility (HET) domains [149]. In the current study, 11 genes encoding heterokaryon incompatibility proteins were up-regulated at some point during the infection with one group induced during the early stages (SS1G_02742, SS1G_02602, SS1G_03889, SS1G_11165 and SS1G_06855) and a second group that was induced during the later stages (SS1G_08974, SS1G_06855, SS1G_12973, SS1G_09167 and SS1G_11315) (Table 8). Some HET domain-containing protein coding genes in S. sclerotiorum are paralogues of HET-E-1 family genes of Podospora anserina [38, 150], but these were not up-regulated in the current study. The role of HET domain-containing proteins in S. sclerotiorum and $B$. cinerea speciation has also been suggested [38]. Some $S$. sclerotiorum orthologues of $A$. nidulans genes involved in mating and fruiting body development, such as SS1G_09861 and SS1G_07526 [38], were also upregulated in the current study. Additionally, SS1G_06124 (transcription factor) and SS1G_00606 (STE 11 kinase), which are also orthologues of $A$. nidulans genes involved 
in mating process signaling pathways [38], were significantly induced in the current study.

Mobilization of storage reserves, including those derived from cell wall turnover and reconstruction, accompanies major morphological phase transitions, such as sclerotial formation. Trehalose is a common storage carbohydrate in fungi and a gene encoding an acid trehalase (SS1G_05192) was induced at the later stages of the infection. Alpha-1,3-glucan (mutan) is a component of the fungal cell wall, but is also considered to be a major energy reserve [151]. Two genes encoding 1,3-alpha-glucanase/mutanase (SS1G_01494 and SS1G_09861) were sharply induced (15 and 30 fold) at $48 \mathrm{hpi}$. The expression of these genes closely coincided with the expression of a wide variety of genes encoding enzymes capable of degrading fungal cell walls, including chitinases, chitin deacetylases, Nacetylglucosaminidases and various endo-glucanases. In another study, $19 \%$ of all S. sclerotiorum genes encoding fungal cell wall degrading enzymes were up-regulated during infection [49]. They hypothesized that these enzymes were involved in cell wall reorganization or rearrangement as the pathogen progressed through different developmental stages.

\section{Secreted effectors}

S. sclerotiorum secretes a large repertoire of various effector proteins that may be involved in aspects of pathogenesis or virulence [44]. Several of these, and others, were found to be up-regulated during infection of B. napus in the current study (Table 9) and are discussed below.
One of the hallmarks associated with S. sclertoriorum infection is the rapid onset of necrosis. Two S. sclerotiorum necrosis and ethylene-inducing protein (NEP) proteins (SsNEP1 and SsNEP2) were characterized by Bashi et al. [30] and their necrosis-inducing activity demonstrated. In that study, both genes were induced at the mid to later times in the infection with $S S N E P 2$ being expressed at much higher levels than SsNEP1. This is in agreement with the current study, the SsNEP2 gene (SS1G_11912) was induced at 24 and 48 hpi. Orthologues of these genes are also present in B. cinerea (BCNEP1 and BcNEP2) and both proteins are capable of inducing necrosis in the host plants [152]. Cerato-plantanins are small, hydrophobic, secreted proteins found in many fungal phyto-pathogens and have been shown to induce plant defenses leading to systemic acquired resistance [153]. In B. cinerea, cerato-platanin is one of the most abundant secreted proteins and elicits a strong hypersensitive response in the host plant leading to localized necrotic lesions [154]. An S. sclerotiorum gene encoding cerato-platanin (SS1G_10096) was up-regulated at both the early and later stages of the infection in the current study. SsSSVP1 (SS1G_02068) encodes a small secreted, cysteine-rich protein that induces plant cell death by interfering with host energy metabolism and, as such, plays an important role in virulence in S. sclerotiorum [155]. In the current study, SS1G_02068 was up-regulated 21 -fold at $48 \mathrm{hpi}$. In the S. sclerotiorum- A. thaliana interaction, SSSSVP1 showed significant up-regulation starting from 3 hpi and slowly increased from 6 to 12 hpi [155], suggesting that the expression pattern of this gene could be host-dependent. As noted above, several hydrolytic enzymes, including certain polygalacturonases $[54,55]$ and xylanses [57], are also potent inducers of host necrosis.

Table 9 Description and expression of up-regulated genes encoding putative effector proteins

\begin{tabular}{|c|c|c|c|c|c|c|c|}
\hline \multirow[t]{2}{*}{ Gene ID } & \multirow[t]{2}{*}{ Description $^{a}$} & \multicolumn{6}{|c|}{ 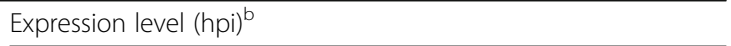 } \\
\hline & & 1 & 3 & 6 & 12 & 24 & 48 \\
\hline SS1G_08557 & salicylate hydroxylase & 4.1 & 5.4 & 5.1 & 3.6 & 5.1 & 10.8 \\
\hline SS1G_00849 & 22kda glycoprotein (AltA-1 allergen) & 7.4 & 3.2 & - & - & - & 7.1 \\
\hline SS1G_10096 & cerato-platanin (PF07249) & 3.8 & 3.7 & - & - & - & 3.3 \\
\hline SS1G_07295 & cfem domain-containing protein & 2 & - & - & - & 3.9 & 4.8 \\
\hline SS1G_12336 & chitin binding protein & - & 3.3 & - & - & - & - \\
\hline SS1G_12509 & LysM domain protein & - & - & - & 3.8 & 11.8 & 35.4 \\
\hline SS1G_03611 & cysteine-rich protein & - & - & - & - & 85.1 & 247 \\
\hline SS1G_11912 & npp1 domain protein (NEP2) & - & - & - & - & 5.8 & 8.2 \\
\hline SS1G_03282 & serine protease inhibitor & - & - & - & - & - & 2.2 \\
\hline SS1G_02904 & cyanoVirin-N homology (SsCVNH) & - & - & - & - & - & 3.9 \\
\hline SS1G_00263 & protein unique to S. sclerotiorum and B. cinerea (Ssv263) & - & - & - & - & - & 49.2 \\
\hline SS1G_02068 & predicted protein (SSSSVP1) & - & - & - & - & - & 21.5 \\
\hline
\end{tabular}

${ }^{a}$ Annotation based on the presence of conserved PFAM domains and BLAST reports

${ }^{b}$ Fold change relative to $0 \mathrm{~h}$ post inoculation (hpi). (-) No significant change in expression

More information about the genes can be found in Additional file 3: Table S3 
A gene encoding a cysteine-rich protein with a CFEM (common fungal extracellular membrane) domain (SS1G_07295) did not show significant induction in a previous study conducted on a number of host plants [44], whereas in the current study it was induced between 2 and 4.8 fold throughout the course of the infection supporting the notion that expression of effector genes in S. sclerotiorum may be hostdependent. In Magnaporthe grisea, the CFEM protein Pth11 is involved in appressorium development [156], while in Candida species CFEM proteins were involved in biofilm formation and iron acquisition [157]. SsCVNH (SS1G_02904), which encodes a small, cysteine-rich, secreted protein with a CyanoVirin-N Homology $(\mathrm{CVNH})$ domain, was previously predicted to be a candidate effector of S. sclerotiorum [44], and was shown to be important for infection, sclerotial development and growth of S. sclerotiorum [49]. SsCVNH was up-regulated at 48 hpi in the current study. Similarly, Lyu et al. [49] showed that $\mathrm{SsCVNH}_{\mathrm{N}}$ was significantly up-regulated during the initial stages of sclerotial development occurring at 3 days post-inoculation. The induction of $\mathrm{SsCVNH}$ coincided with that of Pac1 which is also involved in sclerotial development through OA-mediated $\mathrm{pH}$ reduction, suggesting that the expression of $S s C V N H$ might also be $\mathrm{pH}$ dependent. A gene encoding another cysteine-rich protein (SS1G_03611) was one of the most highly up-regulated genes detected in the current study and exhibited a 247 fold increase in expression at $48 \mathrm{hpi}$, while a gene encoding a protein unique to $S$. sclerotiorum and $B$. cinerea (SS1G_00263) was induced 49-fold at this time. The protein encoded by SS1G_00849 had none of the domains associated with fungal effectors; however, it is an orthologue of Colletotrichum hingginsianum effector candidate 91 (CHEC91) [158] and analogous to the Alternaria alternate AltA-1 allergen [44]. SS1G_00849 was significantly induced at 1-3 and 48 hpi in the current study supporting the view that it may also be a S. sclerotiorum effector.

Some effectors facilitate infection by abrogating the ability of the host to deploy appropriate defense responses. Lysin motif (LysM) effectors interfere with host detection of the pathogen by binding to and masking fungal cell wall-derived chitin fragments that would normally induce host defense responses [159]. A gene encoding a LysM protein (SS1G_12509) was up-regulated during the mid to later stages of the infection, while a gene encoding another chitin-binding protein (SS1G_12336) was up-regulated at $3 \mathrm{hpi}$. Salicylic acid is a signaling molecule required for the induction of plant defenses in response to many biotic and abiotic stresses. Enzymes that degrade salicylic acid are released by some fungal endophytes to suppress the deployment of such defenses [160]. In the current study, a gene encoding salicylate hydroxylase (SS1G_08557) was induced very early in the infection and remained up- regulated throughout, although this gene does not have signal peptide and it might be secreted through an alternative endoplasmic reticulum/Golgi-independent protein secretion mechanism.

\section{Conclusions}

S. sclerotiorum deploys a wide variety of factors and complex strategies to establish disease and complete the infection of the host plant. Soon after encountering the surface of a suitable host plant, the pathogen releases enzymes that begin to digest the cuticle. The induction of SsCuta and genes encoding related cutinases/lipases at the earliest stages of the infection supports their role in cuticle penetration. The enzymatic degradation of other plant surface polymers and polysaccharides is also required for successful penetration and is carried out by an armory of hydrolytic enzymes, which were induced from 1 to 12 hpi in this study. These enzymes are released from the base of infection cushions which apply pressure to assist cuticle penetration. Induction of orthologous genes involved in appressorium formation in other fungi, such as mas 2 and mas3 [38, 141] and SsGgt1 [24] during the early stages of infection in the current study supports their role in the production of penetration-associated structures.

During penetration and subsequent proliferation through the host, the fungus must sequentially breach various layers of plant barriers. To do so, it releases a cocktail of hydrolytic enzymes, detoxification systems and effector proteins. A plethora of genes encoding hydrolytic enzymes were induced concurrent with cell wall and host plant tissue disruption and are required to release nutrients to facilitate spread of the pathogen. Noxious compounds liberated as a form of plant defense or through the activities of the pathogen itself must also be detoxified. This study revealed that S. sclerotiorum induces the expression of genes encoding a wide variety of $A B C$ and MFS transporters, cytochrome 450 enzymes, GSTs., etc, during the infection that may allow it to contend with various host plant defense mechanisms and toxins.

Recent studies have identified a brief biotrophic phase within the apoplastic space immediately after cuticle penetration [3]. During infection of B. napus in the current study, this biotrophic stage might occur between 12 and 24 hpi since biotrophy-related effector genes, such as those encoding the LysM domain protein and salicylate hydroxylase, were up-regulated during this period. These proteins may assist with suppression of host-pathogen recognition and defense systems. However, the genes encoding SSITL [26] and chorismate mutase (SsCM1) [27], which also help to suppress plant defense responses during the biotrophic phase, were not induced in the current study. This discrepancy might indicate that $S$. sclerotiorum is 
armed with alternate, unelucidated, strategies to suppress host defenses to establish a biotrophic phase in different host plants.

The appearance of necrotic lesions at $24 \mathrm{hpi}$ indicated that initial penetration and the biotrophic phase had ended by this time. The onset of the necrotrophic stage requires that a different set of genes be expressed, including those encoding hydrolytic enzymes, enzymes involved in secondary metabolite synthesis or toxins to trigger host programmed cell death. Numerous genes encoding enzymes involved in the synthesis of polyketides and non-ribosomal peptides were up-regulated throughout the infection, but more so during the later stages. Previous studies also suggested that the induction of genes encoding NEP proteins coincides with the beginning of the necrotrophic phase [161]. The expression of SsNEP2 in the current study was induced beginning at 24 hpi, confirming that S. sclerotiorum had switched to the necrotrophic stage around this time.

The acidic environment resulting from OA accumulation is a critical step during the necrotrophic phase of $S$. sclerotiorum. In the present study, the genes encoding $\mathrm{Pac1}$ and subsequently $\mathrm{OAH}$ which are indirectly and directly involved in OA biosynthesis, respectively, were up-regulated at $48 \mathrm{hpi}$, supporting the notion that OA accumulates during the necrotrophic phase of infection. The acidic environment produced by OA stimulates hydrolytic enzyme production, specifically SsPG1 [7, 112, 118, 119]. Induction of acpl, whose expression is sensitive to $\mathrm{pH}$, is also regulated by Pac1 [21]. Interestingly, the concurrent expression of $O A H$ and the gene encoding oxalate decarboxylase suggests that a balance between biosynthesis and decomposition of $\mathrm{OA}$ is required for tight regulation of OA levels through the course of infection [38]. In addition to its role in regulating the transition to the necrotrophic phase, $\mathrm{OA}$ also plays a role in suppression of the oxidative burst in the host plant during the early stages of the infection [15] and may therefore contribute to the establishment of a biotrophic phase. However, $O A H$ expression was not induced during the early stages of infection in the current study suggesting that an alternative pathway for $\mathrm{OA}$ biosynthesis independent of $\mathrm{OAH}$ exists or more likely that a basal amount of OA is sufficient for suppression of oxidative burst.

In addition to the well-known effectors that have been mentioned above, a number of genes encoding other $S$. sclerotiorum effectors, including SsBi1 [20], SsCaf1 [25], SCat1 [18], SsSodI [19] and SsPemG1 [29] were expressed, but not significantly induced in the current study. The discrepancy might be due to host and isolatedependent differences in the expression of these genes or differences in experimental design.

In summary, the current study revealed a vast set of genes encoding various hydrolytic enzymes, enzymes involved in secondary methobolite biosynthesis, proteins associated with detoxification systems and effector proteins that collectively facilitate the infection of $B$. napus by $S$. sclerotiorum. The present investigation gives a global view of the gene expression of $S$. sclerotiorum as it infects $B$. napus and provides a baseline for further characterization of important genes involved in the S. sclerotiorum- B. napus and other host molecular interactions.

\section{Additional files}

Additional file 1: Table S1. Oligonucleotides used for ddPCR. Sequences of the forward $(F)$ and reverse $(R)$ primers used for droplet digital PCR (ddPCR) to examine expression of select Sclerotinia sclerotiorum genes during infection of Brassica napus. (DOCX 17 kb)

Additional file 2: Table S2. Output summary generated by $\mathrm{CLC}$ Genomics Workbench of mapped Illumina reads against S. sclerotiorum isolate 1980 reference transcriptome. Account of the total and mapped Illumina reads generated from three biological replicates of libraries generated from Sclerotinia sclerotiorum mycelia at various times during infection of Brassica napus. (DOCX $22 \mathrm{~kb}$ )

Additional file 3: Table S3. List of up-regulated genes with BLAST2GO annotation. Description of all genes that were up-regulated in Sclerotinia sclerotiorum at various times during infection of Brassica napus. (XLSX $413 \mathrm{~kb}$ )

Additional file 4: Figure S1. Clustering of differentially expressed genes based on expression patterns relative to time of inoculation (time 0 ). Increasing intensity indicates greater fold change (red = positive; green = negative) for individual genes. The expression of Sclerotinia sclerotiorum genes at various times during infection of Brassica napus were used for cluster analysis and a heat map was generated. (PDF $208 \mathrm{~kb}$ )

\section{Abbreviations}

ABC: ATP-binding cassette; CAZymes: Carbohydrate-active enzymes; CE: Carbohydrate esterase; CFEM: Common fungal extracellular membrane; CWDE: Cell wall degrading enzymes; ddPCR: droplet digital PCR; DMI: Demethylation inhibitor; EST: Expressed sequence tag; FDR: False discovery rate; GH: Glycoside hydrolase; GO: Gene ontology; GST: Glutathione S-transferase; MFS: Major facilitator superfamily; NADPH: Nicotinamide adenine dinucleotide phosphate; NRPS: Nonribosomal peptide synthase; OA: Oxalic acid; OAH: Oxaloacetate acetyl hydrolase; PCA: Principal component analysis; PCD: Programmed cell death; PG: Polygalacturonase; PKS: Polyketide synthase; ROS: Reactive oxygen species; RT: Reverse transcriptase; Ss1980: Sclerotinia sclerotiorum isolate 1980; TF: Transcription factor; TMM: Trimmed means of means

Acknowledgements

We thank Diana Bekkaoui for her technical assistance.

\section{Funding}

This study was funded by SaskCanola and the Government of Canada through the Developing Innovative Agri-Products program. The funding body did not participate in the design of the study, in the collection, analysis, and interpretation of data or in the writing of the manuscript.

\section{Availability of data and materials}

The RNA-Seq data of this study is available in NCBI's Gene Expression Omnibus and is accessible through GEO series accession number GSE83935. Plant material used in this study (Brassica napus DH12075) is a doubled-haploid line developed by Agriculture and Agri-Food Canada (Gerhard Rakow and Ginette Sequin-Swartz) from a cross between B. napus cv. Westar and cv. Cresor. Permissions and/or licences to acquire and use this material for research or commercial purposes may be obtained from AAFC. 


\section{Authors' contributions}

SS, DH, YW and HB designed the study. SS performed the experiments. SS, CC and SR analyzed the RNA-Seq data. SS and DH wrote the manuscript. DH, YW and HB revised the manuscript. All authors read and approved the final manuscript.

\section{Competing interests}

The authors declare that they have no competing interests.

\section{Consent for publication}

Not applicable.

\section{Ethics approval and consent to participate}

Experimental research on plants in this study complied with AAFC institutional guidelines. The research did not involve field studies or the collection of wild specimens.

\section{Publisher's Note}

Springer Nature remains neutral with regard to jurisdictional claims in published maps and institutional affiliations.

\section{Author details}

${ }^{1}$ Agriculture and Agri-Food Canada, 107 Science Place, Saskatoon, SK S7N 0X2, Canada. ${ }^{2}$ Department of Biology, University of Saskatchewan, Saskatoon, Canada. ${ }^{3}$ Department of Food and Bioproduct Sciences, University of Saskatchewan, Saskatoon, Canada.

\section{Received: 14 October 2016 Accepted: 18 March 2017} Published online: 29 March 2017

\section{References}

1. Boland GJ, Hall R. Index of plant hosts of Sclerotinia sclerotiorum. Can J Plant Pathol. 1994;16:93-108

2. Hegedus DD, Rimmer SR. Sclerotinia sclerotiorum: When "to be or not to be" a pathogen? Fed Eur Microbiol Soc Microbiol Lett. 2005:251:177-84.

3. Kabbage M, Yarden O, Dickman MB. Pathogenic attributes of Sclerotinia sclerotiorum: Switching from a biotrophic to necrotrophic lifestyle. Plant Sci. 2015;233:53-60.

4. Bashi ZD, Rimmer SR, Khachatourians GG, Hegedus DD. Factors governing the regulation of Sclerotinia sclerotiorum cutinase $A$ and polygalacturonase during different stages of infection. Can J Microbiol. 2012;58:605-16.

5. Vautard-Mey G, Cotton P, Fèvre M. Expression and compartmentation of the glucose repressor CRE1 from the phytopathogenic fungus Sclerotinia sclerotiorum. Eur J Biochem. 1999:266:252-9.

6. Jurick II WM, Rollins JA. Deletion of the adenylate cyclase (sac1) gene affects multiple developmental pathways and pathogenicity in Sclerotinia sclerotiorum. Fungal Genet Biol. 2007;44:521-30.

7. Rollins JA. The Sclerotinia sclerotiorum pac1 gene is required for sclerotial development and virulence. Mol Plant-Microbe Interact. 2003;16:785-95.

8. Kim H, Chen C, Kabbage M, Dickman MB. Identification and characterization of Sclerotinia sclerotiorum NADPH oxidases. Appl Environ Microbiol. 2011;77:7721-9.

9. Chen C, Dickman MB. CAMP blocks MAPK activation and sclerotial development via Rap-1 in a PKA-independent manner in Sclerotinia sclerotiorum. Mol Microbiol. 2005;55:299-311.

10. Duan Y, Ge C, Liu S, Wang J, Zhou M. A two-component histidine kinase Shk1 controls stress response, sclerotial formation and fungicide resistance in Sclerotinia sclerotiorum. Mol Plant Pathol. 2013;14:708-18.

11. Hegedus DD, Gerbrandt K, Coutu C. The eukaryotic protein kinase superfamily of the necrotrophic fungal plant pathogen, Sclerotinia sclerotiorum. Mol Plant Pathol. 2016;17:634-47.

12. Harel A, Bercovich S, Yarden O. Calcineurin is required for sclerotial development and pathogenicity of Sclerotinia sclerotiorum in an oxalic acidindependent manner. Mol Plant-Microbe Interact. 2006;19:682-93.

13. Erental A, Harel A, Yarden O. Type 2A phosphoprotein phosphatase is required for asexual development and pathogenesis of Sclerotinia sclerotiorum. Mol Plant-Microbe Interact. 2007:20:944-54.

14. Godoy G, Steadman JR, Dickman MB, Dam R. Use of mutants to demonstrate the role of oxalic acid in pathogenicity of Sclerotinia sclerotiorum on Phaseolus vulgaris. Physiol Mol Plant Pathol. 1990;37:179-91.
15. Williams B, Kabbage M, Kim H-J, Britt R, Dickman MB. Tipping the balance: Sclerotinia sclerotiorum secreted oxalic acid suppresses host defenses by manipulating the host redox environment. PLoS Pathog. 2011;7:e1002107.

16. Kim KS, Min J-Y, Dickman MB. Oxalic acid is an elicitor of plant programmed cell death during Sclerotinia sclerotiorum disease development. Mol Plant-Microbe Interact. 2008:21:605-12.

17. Calla B, Blahut-Beatty L, Koziol L, Zhang Y, Neece DJ, Carbajulca D, Garcia A, Simmonds DH, Clough SJ. Genomic evaluation of oxalate-degrading transgenic soybean in response to Sclerotinia sclerotiorum infection. Mol Plant Pathol. 2014;15:563-75.

18. Yarden $\mathrm{O}$, Veluchamy S, Dickman MB, Kabbage M. Sclerotinia sclerotiorum catalase SCAT1 affects oxidative stress tolerance, regulates ergosterol levels and controls pathogenic development. Physiol Mol Plant Pathol. 2014;85:34-41.

19. Xu L, Chen W. Random T-DNA mutagenesis identifies a Cu/Zn superoxide dismutase gene as a virulence factor of Sclerotinia sclerotiorum. Mol Plant-Microbe Interact. 2013;26:431-41

20. Yu Y, Xiao J, Yang Y, Bi C, Qing L, Tan W. Ss-Bi1 encodes a putative BAX inhibitor-1 protein that is required for full virulence of Sclerotinia sclerotiorum. Physiol Mol Plant Pathol. 2015:90:115-22.

21. Poussereau N, Creton S, Rascle C, Fevre M. Regulation of acp1, encoding a non-aspartyl acid protease expressed during pathogenesis of Sclerotinia sclerotiorum. Microbiology. 2001;147:717-26.

22. Poussereau N, Gente S, Rascle C, Billon-Grand G, Fevre M. AspS encoding an unusual aspartyl protease from Sclerotinia sclerotiorum is expressed during phytopathogenesis. FEMS Microbiol. 2001;194:27-32.

23. Li R, Rimmer R, Buchwaldt L, Sharpe AG, Séguin-Swartz G, Hegedus DD. Interaction of Sclerotinia sclerotiorum with Brassica napus: cloning and characterization of endo- and exo-polygalacturonases expressed during saprophytic and parasitic modes. Fungal Genet Biol. 2004;41:754-65.

24. Li M, Liang X, Rollins JA. Sclerotinia sclerotiorum Y-glutamyl transpeptidase (Ss-Ggt1) is required for regulating glutathione accumulation and development of sclerotia and compound appressoria. Mol Plant-Microbe Interact. 2012;25:412-20

25. Xiao X, Xie J, Cheng J, Li G, Yi X, Jiang D, Fu Y. Novel secretory protein Ss-Caf1 of the plant-pathogenic fungus Sclerotinia sclerotiorum is required for host penetration and normal sclerotial development. Mol Plant-Microbe Interact. 2014;27:40-55.

26. Zhu W, Wei W, Fu Y, Cheng J, Xie J, Li G, Yi X, Kang Z, Dickman MB, Jiang D. A secretory protein of necrotrophic fungus Sclerotinia sclerotiorum that suppresses host resistance. PLoS ONE. 2013;8:e53901.

27. Kabbage M, Williams B, Dickman MB. Cell death control: The interplay of apoptosis and autophagy in the pathogenicity of Sclerotinia sclerotiorum. PLoS Pathog. 2013;9:e1003287.

28. Pedras MSC, Ahiahonu PWK, Hossain M. Detoxification of the cruciferous phytoalexin brassinin in Sclerotinia sclerotiorum requires an inducible glucosyltransferase. Phytochemistry. 2004;65:2685-94.

29. Pan Y, Xu Y, Li X, Yao C, Gao Z. SsPemG1 encodes an elicitor-homologous protein and regulates pathogenicity in Sclerotinia sclerotiorum. Physiol Mol Plant Pathol. 2015:92:70-8.

30. Bashi ZD, Hegedus DD, Buchwaldt L, Rimmer SR, Borhan MH. Expression and regulation of Sclerotinia sclerotiorum necrosis and ethylene-inducing peptides (NEPs). Mol Plant Pathol. 2010;11:43-53.

31. Zhang H, Wu Q, Cao S, Zhao T, Chen L, Zhuang P, Zhou X, Gao Z. A nove protein elicitor (SsCut) from Sclerotinia sclerotiorum induces multiple defense responses in plants. Plant Mol Biol. 2014:86:495-511.

32. Liang Y, Yajima W, Davis MR, Kav NNV, Strelkov SE. Disruption of a gene encoding a hypothetical secreted protein from Sclerotinia sclerotiorum reduces its virulence on canola (Brassica napus). Can J Plant Pathol. 2013;35:46-55.

33. Li R, Rimmer R, Buchwaldt L, Sharpe AG, Séguin-Swartz G, Coutu C, Hegedus DD. Interaction of Sclerotinia sclerotiorum with a resistant Brassica napus cultivar: expressed sequence tag analysis identifies genes associated with fungal pathogenesis. Fungal Genet Biol. 2004;41:735-53.

34. Sexton AC, Cozijnsen AJ, Keniry A, Jewell E, Love CG, Batley J, Edwards D, Howlett BJ. Comparison of transcription of multiple genes at three developmental stages of the plant pathogen Sclerotinia sclerotiorum. FEMS Microbiol. 2006:258:150-60.

35. Liang Y, Rahman MH, Strelkov SE, Kav NNV. Developmentally induced changes in the sclerotial proteome of Sclerotinia sclerotiorum. Fungal Biol. 2010;114:619-27.

36. Zhao J, Buchwaldt L, Rimmer SR, Sharpe A, Mcgregor L, Bekkaoui D, Hegedus D. Patterns of differential gene expression in Brassica napus cultivars infected with Sclerotinia sclerotiorum. Mol Plant Pathol. 2009;10:635-49. 
37. Joshi RK, Megha S, Rahman MH, Basu U, Kav NNV. A global study of transcriptome dynamics in canola (Brassica napus L.) responsive to Sclerotinia sclerotiorum infection using RNA-Seq. Gene. 2016;590:57-67.

38. Amselem J, Cuomo CA, van Kan JAL, Viaud M, Benito EP, Couloux A, et al. Genomic analysis of the necrotrophic fungal pathogens Sclerotinia sclerotiorum and Botrytis cinerea. PLoS Genet. 2011;7:e1002230.

39. Zhuang X, McPhee KE, Coram TE, Peever TL, Chilvers MI. Rapid transcriptome characterization and parsing of sequences in a non-model host-pathogen interaction; pea-Sclerotinia sclerotiorum. BMC Genomics. 2012;26:668

40. Oliveira MB, de Andrade RV, Grossi-de-Sa MF, Petrofeza S. Analysis of genes that are differentially expressed during the Sclerotinia sclerotiorum-Phaseolus vulgaris interaction. Front Microbiol. 2015;6:1162.

41. Orshinsky AM, Hu J, Opiyo SO, Reddyvari-Channarayappa V, Mitchell TK, Boehm MJ. RNA-Seq analysis of the Sclerotinia homoeocarpa-creeping bentgrass pathosystem. PLoS ONE. 2012;7:e41150.

42. Yajima W, Kav NNV. The proteome of the phytopathogenic fungus Sclerotinia sclerotiorum. Proteomics. 2006;6:5995-6007.

43. Heard S, Brown NA, Hammond-Kosack K. An interspecies comparative analysis of the predicted secretomes of the necrotrophic plant pathogens Sclerotinia sclerotiorum and Botrytis cinerea. PLoS ONE. 2015;10:e0130534.

44. Guyon K, Balagué C, Roby D, Raffaele S. Secretome analysis reveals effector candidates associated with broad host range necrotrophy in the fungal plant pathogen Sclerotinia sclerotiorum. BMC Genomics. 2014;15:336.

45. Robinson MD, Oshlack A. A scaling normalization method for differential expression analysis of RNA-seq data. Genome Biol. 2010;11:R25.

46. Myhre S, Tveit H, Mollestad T, Laegreid A. Additional gene ontology structure for improved biological reasoning. Bioinformatics. 2006;22:2020-7.

47. Gioti A, Simon A, Le Pecheur P, Giraud C, Pradier JM, Viaud M, Levis C. Expression profiling of Botrytis cinerea genes identifies three patterns of up-regulation in planta and an FKBP12 protein affecting pathogenicity. J Mol Biol. 2006;358:372-86.

48. De Cremer K, Mathys J, Vos C, Froenicke L, Michelmore RW, Cammue BPA, De Coninck B. RNAseq-based transcriptome analysis of Lactuca sativa infected by the fungal necrotroph Botrytis cinerea. Plant Cell Environ. 2013;36:1992-2007.

49. Lyu X, Shen C, Fu Y, Xie J, Jiang D, Li G, Cheng J. Comparative genomic and transcriptional analyses of the carbohydrate-active enzymes and secretomes of phytopathogenic fungi reveal their significant roles during infection and development. Sci Rep. 2015;5:15565.

50. Yeats TH, Rose JKC. The formation and function of plant cuticles. Plant Physiol. 2013;163:5-20.

51. Reis H, Pfiff S, Hahn M. Molecular and functional characterization of a secreted lipase from Botrytis cinerea. Mol Plant Pathol. 2005;6:257-67.

52. Voigt CA, Schäfer W, Salomon S. A secreted lipase of Fusarium graminearum is a virulence factor required for infection of cereals. Plant J. 2005;42:364-75.

53. Zhao Z, Liu H, Wang C, Xu J-R. Comparative analysis of fungal genomes reveals different plant cell wall degrading capacity in fungi. BMC Genomics. 2013;14:274

54. Bashi ZD, Rimmer SR, Khachatourians GG, Hegedus DD. Brassica napus polygalacturonase inhibitor proteins inhibit Sclerotinia sclerotiorum polygalacturonase enzymatic and necrotizing activities and delay symptoms in transgenic plants. Can J Microbiol. 2013;59:79-86.

55. Kars I, Krooshof GH, Wagemakers L, Joosten R, Benen JAE, van Kan JAL. Necrotizing activity of five Botrytis cinerea endopolygalacturonases produced in Pichia pastoris. Plant J. 2005;43:213-25.

56. ten Have A, Mulder W, Visser J, van Kan JAL. The Endopolygalacturonase gene $B c p g 1$ is required for full virulence of Botrytis cinerea. Mol PlantMicrobe Interact. 1998;11:1009-16.

57. Noda J, Brito N, González C. The Botrytis cinerea xylanase Xyn11A contributes to virulence with its necrotizing activity, not with its catalytic activity. BMC Plant Biol. 2010;10:1-15.

58. Blanco-Ulate B, Morales-Cruz A, Amrine KCH, Labavitch JM, Powell ALT, Cantu D. Genome-wide transcriptional profiling of Botrytis cinerea genes targeting plant cell walls during infections of different hosts. Front Plant Sci. 2014;5:435.

59. Kleman-Leyer KM, Siika-Aho M, Teeri TT, Kirk TK. The cellulases endoglucanase I and cellobiohydrolase II of Trichoderma reesei act synergistically to solubilize native cotton cellulose but not to decrease its molecular size. Appl Environ Microbiol. 1996;62:2883-7.

60. Howell HE. Correlation of virulence with secretion in vitro of three wall-degrading enzymes in isolates of Sclerotinia fructigena obtained after mutagen treatment. J Gen Microbiol. 1975;90:32-40.
61. Sakamoto T, Ishimaru M. Peculiarities and applications of galactanolytic enzymes that act on type I and II arabinogalactans. Appl Microbiol Biotechnol. 2013;97:5201-13.

62. Barros J, Serk H, Granlund I, Pesquet E. The cell biology of lignification in higher plants. Ann Bot. 2015; doi: 10.1093/aob/mcv046.

63. Janusz G, Kucharzyk KH, Pawlik A, Staszczak M, Paszczynski AJ. Fungal laccase, manganese peroxidase and lignin peroxidase: gene expression and regulation. Enzyme Microb Technol. 2013;52:1-12.

64. Movahedi S, Heale JB. The roles of aspartic proteinase and endo-pectin lyase enzymes in the primary stages of infection and pathogenesis of various host tissues by different isolates of Botrytis cinerea Pers ex. Pers Physiol Mol Plant Pathol. 1990;36:303-24.

65. Olivieri F, Eugenia Zanetti M, Oliva CR, Covarrubias AA, Casalongué CA. Characterization of an extracellular serine protease of Fusarium eumartii and its action on pathogenesis related proteins. Eur J Plant Pathol. 2002;108:63-72.

66. Zuccaro A, Lahrmann U, Güldener U, Langen G, Pfiffi S, Biedenkopf D, et al. Endophytic life strategies decoded by genome and transcriptome analyses of the mutualistic root symbiont Piriformospora indica. PLoS Pathog. 2011;7:e1002290.

67. Vardar F, Ünal M. Proteolytic enzymes in plant programmed cell death. Türk Bilim Derlemeler Derg. 2008;1:65-78.

68. Beers EP, Woffenden BJ, Zhao C. Plant proteolytic enzymes: possible roles during programmed cell death. Plant Mol Biol. 2000;44:399-415.

69. Navarre DA, Wolpert TJ. Victorin induction of an apoptotic/senescence-like response in oats. Plant Cell. 1999;11:237-49.

70. Carpita NC, Gibeaut DM. Structural models of primary cell walls in flowering plants: consistency of molecular structure with the physical properties of the walls during growth. Plant J. 1993;3:1-30.

71. Choquer M, Fournier E, Kunz C, Levis C, Pradier J-M, Simon A, Viaud M. Botrytis cinerea virulence factors: new insights into a necrotrophic and polyphageous pathogen. FEMS Microbiol. 2007;277:1-10.

72. Colmenares AJ, Aleu J, Durán-Patrón R, Collado IG, Hernández-Galán R. The putative role of botrydial and related metabolites in the infection mechanism of Botrytis cinerea. J Chem Ecol. 2002;28:997-1005.

73. Siewers V, Viaud M, Jimenez-Teja D, Collado IG, Gronover CS, Pradier J-M, Tudzynski B, Tudzynski P. Functional analysis of the cytochrome P450 monooxygenase gene bcbot1 of Botrytis cinerea indicates that botrydial is a strain-specific virulence factor. Mol Plant-Microbe Interact. 2005;18:602-12.

74. Kelkar HS, Skloss TW, Haw JF, Keller NP, Adams TH. Aspergillus nidulans stcL encodes a putative cytochrome P-450 monooxygenase required for bisfuran desaturation during aflatoxin/sterigmatocystin biosynthesis. J Biol Chem. 1997;272:1589-94.

75. Cresnar B, Petric S. Cytochrome P450 enzymes in the fungal kingdom. Biochim Biophys Acta. 1814;2011:29-35.

76. Dalmais B, Schumacher J, Moraga J, LE Pecheur P, Tudzynski B, Collado IG, Viaud M. The Botrytis cinerea phytotoxin botcinic acid requires two polyketide synthases for production and has a redundant role in virulence with botrydial. Mol Plant Pathol. 2011;12:564-79.

77. Pedras MSC, Ahiahonu PWK. Phytotoxin production and phytoalexin elicitation by the phytopathogenic fungus Sclerotinia sclerotiorum. J Chem Ecol. 2004;30:2163-79.

78. Kennedy J, Auclair K, Kendrew SG, Park C, Vederas JC, Hutchinson CR. Modulation of polyketide synthase activity by accessory proteins during lovastatin biosynthesis. Science. 1999;284:1368-72.

79. Oide S, Moeder W, Krasnoff S, Gibson D, Haas H, Yoshioka K, Turgeon BG. NPS6, encoding a nonribosomal peptide synthetase involved in siderophore-mediated iron metabolism, is a conserved virulence determinant of plant pathogenic ascomycetes. Plant Cell. 2006;18:2836-53.

80. Schrettl M, Bignell E, Kragl C, Joechl C, Rogers T, Arst HN, Haynes K, Haas H. Siderophore biosynthesis but not reductive iron assimilation is essential for Aspergillus fumigatus virulence. J Exp Med. 2004;200:1213-9.

81. Tripathy BC, Sherameti I, Oelmuller R. Siroheme: an essential component for life on earth. Plant Signal Behav. 2010;5:14-20.

82. Walton JD. HC-toxin. Phytochemistry. 2006;67:1406-13.

83. Pitkin JW, Panaccionet DG, Walton JD. A putative cyclic peptide efflux pump encoded by the TOXA gene of the plant-pathogenic fungus Cochliobolus carbonurn. Microbiology. 1996;142:1557-65.

84. VanEtten H, Temporini E, Wasmann C. Phytoalexin (and phytoanticipin) tolerance as a virulence trait: why is it not required by all pathogens? Physiol Mol Plant Pathol. 2001;59:83-93.

85. Perlin MH, Andrews J, Toh SS. Essential letters in the fungal alphabet: $A B C$ and MFS transporters and their roles in survival and pathogenicity. Adv Genet. 2014;85:201-53. 
86. Stefanato FL, Abou-Mansour E, Buchala A, Kretschmer M, Mosbach A, Hahn M, et al. The ABC transporter BcatrB from Botrytis cinerea exports camalexin and is a virulence factor on Arabidopsis thaliana. Plant J. 2009;58:499-510.

87. Schoonbeek H, Del Sorbo G, De Waard MA. The ABC transporter BcatrB affects the sensitivity of Botrytis cinerea to the phytoalexin resveratrol and the fungicide fenpiclonil. Mol Plant-Microbe Interact. 2001;14:562-71.

88. Schoonbeek H, van Nistelrooy JGM, de Waard MA. Functional analysis of $A B C$ transporter genes from Botrytis cinerea identifies BcatrB as a transporter of eugenol. Eur J Plant Pathol. 2003;109:1003-11.

89. Del Sorbo G, Ruocco M, Schoonbeek H-J, Scala F, Pane C, Vinale F, De Waard MA. Cloning and functional characterization of BcatrA, a gene encoding an $A B C$ transporter of the plant pathogenic fungus Botryotinia fuckeliana (Botrytis cinerea). Mycol Res. 2008;112:737-46.

90. Hayashi K, Schoonbeek H, De Waard MA. Expression of the ABC transporter BcatrD from Botrytis cinerea reduces sensitivity to sterol demethylation inhibitor fungicides. Pestic Biochem Physiol. 2002;73:110-21.

91. Hulvey J, Popko JTJ, Sang H, Berg A, Jung G. Overexpression of ShCYP51B and ShatrD in Sclerotinia homoeocarpa isolates exhibiting practical field resistance to a demethylation inhibitor fungicide. Appl Environ Microbiol. 2012;78:6674-82

92. Makizumi Y, Takeda S, Matsuzaki Y, Nakaune R, Hamamoto H, Akutsu K, Hibi T. Cloning and selective toxicant-induced expression of BMR1 and $B M R 3$, novel $A B C$ transporter genes in Botrytis cinerea. J Gen Plant Pathol. 2002;68:338-41.

93. Hayashi K, Schoonbeek H-J, De Waard MA. Bcmfs1, a novel major facilitator superfamily transporter from Botrytis cinerea, provides tolerance towards the natural toxic compounds camptothecin and cercosporin and towards fungicides. Appl Environ Microbiol. 2002;68:4996-5004.

94. Sexton AC, Minic Z, Cozijnsen AJ, Pedras MSC, Howlett BJ. Cloning, purification and characterisation of brassinin glucosyltransferase, a phytoalexin-detoxifying enzyme from the plant pathogen Sclerotinia sclerotiorum. Fungal Genet Biol. 2009;46:201-9.

95. George HL, VanEtten HD. Characterization of pisatin-inducible cytochrome P450s in fungal pathogens of pea that detoxify the pea phytoalexin pisatin. Fungal Genet Biol. 2001;33:37-48.

96. Han Y, Liu X, Benny U, Kistler HC, VanEtten HD. Genes determining pathogenicity to pea are clustered on a supernumerary chromosome in the fungal plant pathogen Nectria haematococca. Plant J. 2001;25:305-14.

97. Harwood CS, Parales RE. The beta-ketoadipate pathway and the biology of self-identity. Annu Rev Microbiol. 1996;50:553-90.

98. Faber BW, van Gorcom RF, Duine JA. Purification and characterization of benzoate-para-hydroxylase, a cytochrome P450 (CYP53A1), from Aspergillus niger. Arch Biochem Biophys. 2001;394:245-54.

99. Podobnik B, Stojan J, Lah L, Krasevec N, Seliskar M, Rizner TL, Rozman D, Komel R. CYP53A15 of Cochliobolus lunatus, a target for natural antifungal compounds. J Med Chem. 2008;51:3480-6.

100. Yoshida Y. Lanosterol 14a-demethylase (cytochrome P45014DM). In: Schenkman JB, Greim H, editors. Cytochrome P450. Berlin, Heidelberg: Springer Berlin Heidelberg; 1993. p. 627-39.

101. Parks LW, Casey WM. Physiological implications of sterol biosynthesis in yeast. Annu Rev Microbiol. 1995;49:95-116.

102. Luo C-X, Schnabel G. The cytochrome P450 lanosterol 14alpha-demethylase gene is a demethylation inhibitor fungicide resistance determinant in Monilinia fructicola field isolates from Georgia. Appl Environ Microbiol. 2008;74:359-66

103. Frisch T, Motawia MS, Olsen CE, Agerbirk N, Moller BL, Bjarnholt N. Diversified glucosinolate metabolism: biosynthesis of hydrogen cyanide and of the hydroxynitrile glucoside alliarinoside in relation to sinigrin metabolism in Alliaria petiolata. Front Plant Sci. 2015;6:926.

104. Gonzalez-Fernandez R, Aloria K, Valero-Galvan J, Redondo I, Arizmendi JM, Jorrin-Novo JV. Proteomic analysis of mycelium and secretome of different Botrytis cinerea wild-type strains. J Proteomics. 2014;97:195-221.

105. Sexton AC, Howlett BJ. Characterisation of a cyanide hydratase gene in the phytopathogenic fungus Leptosphaeria maculans. Mol Gen Genet. 2000;263:463-70.

106. Riechers DE, Irzyk GP, Jones SS, Fuerst EP. Partial characterization of glutathione S-transferases from wheat (Triticum spp.) and purification of a safener-induced glutathione S-transferase from Triticum tauschii. Plant Physiol. 1997;114:1461-70.

107. Dowd CA, Buckley CM, Sheehan D. Glutathione S-transferases from the white-rot fungus, Phanerochaete chrysosporium. Biochem J. 1997;324:243-8.
108. Prins TW, Wagemakers L, Schouten A, van Kan JAL. Cloning and characterization of a glutathione S-transferase homologue from the plant pathogenic fungus Botrytis cinerea. Mol Plant Pathol. 2000;1:169-78.

109. Mijatovic S. Biochemical characterization of 2-nitropropane dioxygenase from Hansenula MRAKII. M. Sc. thesis, Georgia State University, 2008. http:// scholarworks.gsu.edu/chemistry_theses/8/.

110. Samolski I, de Luis A, Vizcaíno JA, Monte E, Suárez MB. Gene expression analysis of the biocontrol fungus Trichoderma harzianum in the presence of tomato plants, chitin, or glucose using a high-density oligonucleotide microarray. BMC Microbiol. 2009;9:217.

111. Garrido-Sanz D, Meier-Kolthoff JP, Goker M, Martin M, Rivilla R, Redondo-Nieto M Genomic and genetic diversity within the Pseudomonas fluorescens complex. PLOS ONE. 2016:11:e0150183.

112. Favaron F, Sella L, D'Ovidio R. Relationships among endo-polygalacturonase, oxalate, $\mathrm{pH}$, and plant polygalacturonase-inhibiting protein (PGIP) in the interaction between Sclerotinia sclerotiorum and soybean. Mol Plant-Microbe Interact. 2004;17:1402-9.

113. Davidson AL, Blahut-Beatty L, Itaya A, Zhang Y, Zheng S, Simmonds D. Histopathology of Sclerotinia sclerotiorum infection and oxalic acid function in susceptible and resistant soybean. Plant Pathol. 2016;65:878-87.

114. Maxwell DP. Oxalate formation in Whetzelinia sclerotiorum by oxaloacetate acetylhydrolase. Physiol Plant Pathol. 1973;3:279-88.

115. Han Y, Joosten H-J, Niu W, Zhao Z, Mariano PS, McCalman M, et al. Oxaloacetate hydrolase, the C-C bond lyase of oxalate secreting fungi. J Biol Chem. 2007;282:9581-90.

116. Liang X, Liberti D, Li M, Kim Y-T, Hutchens A, Wilson R, Rollins JA. Oxaloacetate acetylhydrolase gene mutants of Sclerotinia sclerotiorum do not accumulate oxalic acid, but do produce limited lesions on host plants. Mol Plant Pathol. 2015;16:559-71.

117. Magro P, Marciano P, di Lenna P. Enzymatic oxalate decarboxylation in isolates of Sclerotinia sclerotiorum. FEMS Microbiol. 1988;49:49-52.

118. Cotton P, Kasza Z, Bruel C, Rascle C, Fèvre M. Ambient pH controls the expression of endopolygalacturonase genes in the necrotrophic fungus Sclerotinia sclerotiorum. FEMS Microbiol. 2003;227:163-9.

119. Rollins JA, Dickman MB. pH Signaling in Sclerotinia sclerotiorum: Identification of a pacC/RIM1 Homolog. Appl Environ Microbiol. 2001;67:75-81.

120. Xu L, Xiang M, White D, Chen W. pH dependency of sclerotial development and pathogenicity revealed by using genetically defined oxalate-minus mutants of Sclerotinia sclerotiorum. Environ Microbiol. 2015;17:2896-909.

121. Liang X, Moomaw EW, Rollins JA. Fungal oxalate decarboxylase activity contributes to Sclerotinia sclerotiorum early infection by affecting both compound appressoria development and function. Mol Plant Pathol. 2015;16:825-36.

122. Apel K, Hirt H. Reactive oxygen species: metabolism, oxidative stress, and signal transduction. Annu Rev Plant Biol. 2004;55:373-99.

123. Baker CJ, Orlandi EW. Active oxygen in plant pathogenesis. Annu Rev Phytopathol. 1995;33:299-321.

124. Foreman J, Demidchik V, Bothwell JHF, Mylona P, Miedema H, Torres MA, et al. Reactive oxygen species produced by NADPH oxidase regulate plant cell growth. Nature. 2003;422:442-6.

125. Segmüller N, Kokkelink L, Giesbert S, Odinius D, van Kan J, Tudzynski P. NADPH oxidases are involved in differentiation and pathogenicity in Botrytis cinerea. Mol Plant-Microbe Interact. 2008;21:808-19.

126. Malagnac $F$, Lalucque $H$, Lepere $G$, Silar P. Two NADPH oxidase isoforms are required for sexual reproduction and ascospore germination in the filamentous fungus Podospora anserina. Fungal Genet Biol. 2004;41:982-97.

127. MacPherson S, Larochelle M, Turcotte B. A fungal family of transcriptional regulators: the zinc cluster proteins. Microbiol Mol Biol Rev. 2006;70:583-604.

128. Schumacher J, de Larrinoa IF, Tudzynski B. Calcineurin-responsive zinc finger transcription factor CRZ1 of Botrytis cinerea is required for growth, development, and full virulence on bean plants. Eukaryot Cell. 2008;7:584-601.

129. Schumacher J, Viaud M, Simon A, Tudzynski B. The Ga subunit BCG1, the phospholipase $\mathrm{C}(\mathrm{BCPLC1})$ and the calcineurin phosphatase co-ordinately regulate gene expression in the grey mould fungus Botrytis cinerea. Mol Microbiol. 2008;67:1027-50

130. Gil-Durán C, Rojas-Aedo JF, Medina E, Vaca I, García-Rico RO, Villagrán S, et al. The $p c z 1$ gene, which encodes a $\mathrm{Zn}(I) 2 C y s 6$ protein, is involved in the control of growth, conidiation, and conidial germination in the filamentous fungus Penicillium roqueforti. PLoS ONE. 2015;10:e0120740.

131. Simon A, Dalmais B, Morgant G, Viaud M. Screening of a Botrytis cinerea onehybrid library reveals a Cys $2 \mathrm{His} 2$ transcription factor involved in the regulation of secondary metabolism gene clusters. Fungal Genet Biol. 2013;52:9-19. 
132. Tanaka C, Izumitsu K. Two-component signaling system in filamentous fungi and the mode of action of dicarboximide and phenylpyrrole fungicides. In: Carisse O, editor. Fungic. 2010. p. 523-38.

133. Schamber A, Leroch M, Diwo J, Mendgen K, Hahn M. The role of mitogen-activated protein (MAP) kinase signalling components and the Ste12 transcription factor in germination and pathogenicity of Botrytis cinerea. Mol Plant Pathol. 2010;11:105-19.

134. Zhao X, Kim Y, Park G, Xu J-R. A mitogen-activated protein kinase cascade regulating infection-related morphogenesis in Magnaporthe grisea. Plant Cell. 2005;17:1317-29.

135. Ding X, Yu Q, Zhang B, Xu N, Jia C, Dong Y, Chen Y, Xing L, Li M. The type II $\mathrm{Ca} 2+$ /calmodulin-dependent protein kinases are involved in the regulation of cell wall integrity and oxidative stress response in Candida albicans. Biochem Biophys Res Commun. 2014;446:1073-8.

136. Pérez-Valle J, Rothe J, Primo C, Martínez Pastor M, Ariño J, Pascual-Ahuir A et al. $\mathrm{Hal} 4$ and $\mathrm{Hal} 5$ Protein Kinases are required for general control of carbon and nitrogen uptake and metabolism. Eukaryot Cell. 2010;9:1881-90.

137. Nishizawa $\mathrm{M}$. The regulators of yeast $\mathrm{PHO}$ system participate in the transcriptional regulation of G1 cyclin under alkaline stress conditions. Yeast. 2015;32:367-78.

138. Johnson C, Kweon HK, Sheidy D, Shively CA, Mellacheruvu D, Nesvizhskii Al, et al. The yeast Sks1p kinase signaling network regulates pseudohyphal growth and glucose response. PLoS Genet. 2014;10:e1004183.

139. Stajich JE, Wilke SK, Ahren D, Au CH, Birren BW, Borodovsky M, et al. Insights into evolution of multicellular fungi from the assembled chromosomes of the mushroom Coprinopsis cinerea (Coprinus cinereus). Proc Natl Acad Sci U S A. 2010;107:11889-94.

140. Zimmermann S, Moelling K. Phosphorylation and regulation of Raf by Akt (protein kinase B). Science. 1999;286:1741-4.

141. Xue C, Park G, Choi W, Zheng L, Dean RA, Xu J-R. Two novel fungal virulence genes specifically expressed in appressoria of the rice blast fungus. Plant Cell. 2002;14:2107-19.

142. Li M, Rollins JA. The development-specific protein (Ssp1) from Sclerotinia sclerotiorum is encoded by a novel gene expressed exclusively in sclerotium tissues. Mycologia. 2009;101:34-43.

143. Lyu X, Shen C, Fu Y, Xie J, Jiang D, Li G, Cheng J. The microbial opsin homolog Sop1 is involved in Sclerotinia sclerotiorum development and environmental stress response. Front Microbiol. 2015;6:1504.

144. Bayram O, Braus G. Coordination of secondary metabolism and development in fungi: the velvet family of regulatory proteins. FEMS Microbiol Rev. 2012:36:1-24.

145. Yu Y, Xiao J, Zhu W, Yang Y, Mei J, Bi C, Qian W, Qing L, Tan W. Ss-Rhs1, a secretory Rhs repeat-containing protein, is required for the virulence of Sclerotinia sclerotiorum. Mol Plant Pathol. 2016; doi: 10.1111/mpp.12459.

146. Qu X, Yu B, Liu J, Zhang X, Li G, Zhang D, et al. MADS-box transcription factor SSMADS is involved in regulating growth and virulence in Sclerotinia sclerotiorum. Int J Mol Sci. 2014;15:8049-62.

147. Kim S, Park S-Y, Kim KS, Rho H-S, Chi M-H, Choi J, et al. Homeobox transcription factors are required for conidiation and appressorium development in the rice blast fungus Magnaporthe oryzae. PLoS Genet. 2009:5:e1000757

148. Antal Z, Rascle C, Cimerman A, Viaud M, Billon-Grand G, Choquer M, Bruel C. The homeobox $\mathrm{BCHOX} 8$ gene in Botrytis Cinerea regulates vegetative growth and morphology. PLOS ONE. 2012;7:44-8.

149. Paoletti $M$, Clavé $C$. The fungus-specific HET domain mediates programmed cell death in Podospora anserina. Eukaryot Cell. 2007:6:2001-8.

150. Paoletti M, Saupe SJ. Fungal incompatibility: Evolutionary origin in pathogen defense? BioEssays. 2009:31:1201-10.

151. Wei $H$, Scherer M, Singh A, Liese R, Fischer R. Aspergillus nidulans alpha-1,3 glucanase (mutanase), mutA, is expressed during sexual development and mobilizes mutan. Fungal Genet Biol. 2001;34:217-27.

152. Schouten A, van Baarlen P, van Kan JAL. Phytotoxic Nep1-like proteins from the necrotrophic fungus Botrytis cinerea associate with membranes and the nucleus of plant cells. New Phytol. 2008;177:493-505.

153. Frías M, Brito N, González C. The Botrytis cinerea cerato-platanin BcSpl1 is a potent inducer of systemic acquired resistance (SAR) in tobacco and generates a wave of salicylic acid expanding from the site of application. Mol Plant Pathol. 2013;14:191-6.

154. Frías M, Gonzalez C, Brito N. BcSpl1, a cerato-platanin family protein, contributes to Botrytis cinerea virulence and elicits the hypersensitive response in the host. New Phytol. 2011;192:483-95.
155. Lyu X, Shen C, Fu Y, Xie J, Jiang D, Li G, Cheng J. A small secreted virulence-related protein is essential for the necrotrophic interactions of Sclerotinia sclerotiorum with its host plants. PLoS Pathog. 2016:12:e1005435.

156. DeZwaan TM, Carroll AM, Valent B, Sweigard JA. Magnaporthe grisea pth11p is a novel plasma membrane protein that mediates appressorium differentiation in response to inductive substrate cues. Plant Cell. 1999;11:2013-30.

157. Ding C, Vidanes GM, Maguire SL, Guida A, Synnott JM, Andes DR, Butler G. Conserved and divergent roles of Bcr1 and CFEM proteins in Candida parapsilosis and Candida albicans. PLoS ONE. 2011;6:e28151.

158. Kleemann J, Rincon-Rivera LJ, Takahara H, Neumann U, van Themaat EVL, van der Does HC, et al. Sequential delivery of host-induced virulence effectors by appressoria and intracellular hyphae of the phytopathogen Colletotrichum higginsianum. PLoS Pathog. 2012;8:e1002643.

159. Mentlak TA, Kombrink A, Shinya T, Ryder LS, Otomo I, Saitoh H, et al. Effector-mediated suppression of chitin-triggered immunity by Magnaporthe oryzae is necessary for rice blast disease. Plant Cell. 2012;24:322-35

160. Ambrose KV, Tian Z, Wang Y, Smith J, Zylstra G, Huang B, Belanger FC. Functional characterization of salicylate hydroxylase from the fungal endophyte Epichloë festucae. Nature. 2015;5:10939.

161. Qutob D, Kamoun S, Gijzen M. Expression of a Phytophthora sojae necrosis-inducing protein occurs during transition from biotrophy to necrotrophy. Plant J. 2002;32:361-73.

\section{Submit your next manuscript to BioMed Central and we will help you at every step:}

- We accept pre-submission inquiries

- Our selector tool helps you to find the most relevant journal

- We provide round the clock customer support

- Convenient online submission

- Thorough peer review

- Inclusion in PubMed and all major indexing services

- Maximum visibility for your research

Submit your manuscript at www.biomedcentral.com/submit

) Biomed Central 